\title{
Weyl metrics and Wiener-Hopf factorization
}

\author{
P. Aniceto, M.C. Câmara, G.L. Cardoso and M. Rosselló \\ Center for Mathematical Analysis, Geometry and Dynamical Systems, \\ Department of Mathematics, Instituto Superior Técnico, Universidade de Lisboa, \\ Av. Rovisco Pais, 1049-001 Lisboa, Portugal \\ E-mail: pedro.aniceto@tecnico.ulisboa.pt, \\ cristina.camara@tecnico.ulisboa.pt, \\ gabriel.lopes.cardoso@tecnico.ulisboa.pt, \\ martirossello@tecnico.ulisboa.pt
}

ABSTRACT: We consider the Riemann-Hilbert factorization approach to the construction of Weyl metrics in four space-time dimensions. We present, for the first time, a rigorous proof of the remarkable fact that the canonical Wiener-Hopf factorization of a matrix obtained from a general (possibly unbounded) monodromy matrix, with respect to an appropriately chosen contour, yields a solution to the non-linear gravitational field equations. This holds regardless of whether the dimensionally reduced metric in two dimensions has Minkowski or Euclidean signature. We show moreover that, by taking advantage of a certain degree of freedom in the choice of the contour, the same monodromy matrix generally yields various distinct solutions to the field equations. Our proof, which fills various gaps in the existing literature, is based on the solution of a second Riemann-Hilbert problem and highlights the deep role of the spectral curve, the normalization condition in the factorization and the choice of the contour. This approach allows us to construct explicit solutions, including new ones, to the non-linear gravitational field equations, using simple complex analytic results.

KEywords: 2D Gravity, Black Holes, Integrable Field Theories, Sigma Models

ARXIV EPRINT: 1910.10632 


\section{Contents}

1 Introduction $\quad 2$

2 Summary of the main results 4

3 Preliminary results $\quad 8$

3.1 Properties of $\varphi \in \mathcal{T}$

3.2 Contour properties 11

3.3 Affine transformations 11

4 The Breitenlohner-Maison linear system 12

5 Monodromy matrix $\quad 14$

6 Canonical factorization gives a solution to the BM linear system $\quad 16$

7 Meromorphic factorizations: a case study 23

8 The Schwarzschild monodromy matrix 26

$8.1 \sigma=1 \quad 28$

$8.2 \quad \sigma=-1 \quad 31$

8.2.1 Region $I \quad 33$

8.2.2 Extending solutions: the interior region of the Schwarzschild solution 36

$\begin{array}{lll}8.2 .3 & \text { Regions } A \text { and } B & 38\end{array}$

9 The monodromy matrix with $\epsilon=0 \quad 41$

$9.1 \sigma=1 \quad 42$

$9.2 \quad \sigma=-1 \quad 43$

10 Solutions with two Killing horizons 45

A Expressions for $\tilde{\boldsymbol{P}}>\tilde{Q}>0 \quad 49$

B Gluing solutions along the lines $\rho= \pm v+m$

C $A$-metrics 


\section{Introduction}

The field equations of gravitational theories in $D$ space-time dimensions are a system of non-linear PDE's for the space-time metric which are, in general, very difficult to solve. Exact solutions can however be found under simplifying assumptions, for instance spherical symmetry. One such exact solution, of significant physical and mathematical interest, is the well-known Schwarzschild solution which, in $D=4$ dimensions in coordinates $(t, r, \theta, \phi)$, takes the form

$$
d s_{4}^{2}=-\left(1-\frac{2 m}{r}\right) d t^{2}+\left(1-\frac{2 m}{r}\right)^{-1} d r^{2}+r^{2}\left(d \theta^{2}+\sin ^{2} \theta d \phi^{2}\right),
$$

where $m \in \mathbb{R}^{+}, r>0,0<\theta<\pi$ and $0 \leq \phi<2 \pi$.

By restricting to the subspace of solutions that only depend on two of the $D$ spacetime coordinates, various approaches to solving the field equations become available (see for instance [1] for a recent survey thereof). In this paper, we focus on the Riemann-Hilbert approach, which can be applied to gravitational theories that satisfy certain requirements, see for instance [2-6] and references therein. This approach is remarkable in that it allows us to obtain explicit solutions to the reduced field equations through the appropriate factorization of a matrix function which depends on a complex variable $\tau$ that does not appear in the original problem. In this factorization, the space-time coordinates are taken as parameters, thus playing the role of constants. However, to this date, a clear and rigorous proof that, under very general assumptions, this type of matrix factorization always yields a solution to the field equations has not been given. One of the goals of this paper is to provide such a proof. We illustrate the power of this approach by showing that all type $A$ space-time metrics $[7,8]$, which includes the Schwarzschild solution, can be obtained from a single class of diagonal matrices, and by presenting new solutions to the gravitational field equations that we believe would be difficult to obtain through other methods.

Let us briefly summarise the novel aspects discussed in this paper: we give a clear and rigorous proof that, under very general and natural assumptions, the canonical WienerHopf factorization of a so-called monodromy matrix always yields a solution to the gravitational field equations; we show that by choosing different factorization contours one such monodromy matrix gives rise to several distinct solutions to the field equations; we discuss the intricacy of reconstructing the interior region of the Schwarzschild solution from factorization data, which requires gluing together solutions by means of affine transformations; we perform a case study of the relation between meromorphic (as opposed to canonical) factorizations of a monodromy matrix and solutions to the gravitational field equations; we obtain explicit solutions that, to the best of our knowledge, are new.

Let us now give a few more details.

When reducing to two dimensions, there are two distinct cases to consider, which we will distinguish through a parameter $\sigma$ : either all the directions over which one reduces are space-like $(\sigma=-1)$, or one of them is time-like and the others are space-like $(\sigma=1)$.

In this paper, for the first time, a unified Riemann-Hilbert approach to both cases is presented. Namely, we give a rigorous proof that the canonical Wiener-Hopf factorization 
(defined in section 2) of a general monodromy matrix $\mathcal{M}$, with respect to an appropriately chosen contour $\Gamma$ in the complex plane, always yields exact solutions to the gravitational field equations, for $\sigma= \pm 1$. Note that a Wiener-Hopf factorization must be defined with respect to a given contour [9-11].

This proof, which is based on the formulation and solution of another associated Riemann-Hilbert problem, highlights the power of the Riemann-Hilbert approach and clarifies the roles played by the so-called spectral curve and the properties of the Wiener-Hopf factorization. In this way we revisit and generalize the results obtained in [2-5], also filling a few gaps in the presentation given there.

As a result one finds, in particular, that from rational $n \times n$ monodromy matrices $\mathcal{M}$, whose canonical Wiener-Hopf factorization can be constructed explicitly and in a computationally simple manner, one obtains explicit exact solutions that would be very difficult to obtain through other approaches. This is the case of the novel solutions presented in [6], whose construction, based on the Riemann-Hilbert approach of [5], is hereby rigorously justified. Using our improved understanding of the role of the factorization contour, we return to one of the new solutions obtained in [6], which was restricted to a certain domain in space-time. Here we complete its analysis, discuss its meaning and properties, and we also consider other ranges of parameters.

We also show in this paper that by taking advantage of the possible choices of factorization contours $\Gamma$ and appropriate changes of coordinates, each monodromy matrix gives rise not to one solution, but to a whole class of exact solutions. We illustrate this surprising result by showing that from the canonical Wiener-Hopf factorization of a rational diagonal matrix of a very simple kind that is easily factorizable, one obtains a wide class of metrics that includes all type $A$ space-time metrics, a cosmological Kasner solution and the Rindler metric, as well as solutions whose metric tensor is continuous but not smooth due to the presence of null hypersurfaces $[7,8,12,13]$. This wide class includes the solutions that describe the exterior and the interior regions of the Schwarzschild black hole. In doing so, we moreover put in evidence the essential difference between the case $\sigma=1$ and $\sigma=-1$. Previous recent work that addresses this difference includes [14, 15].

This paper is organized as follows. We chose to keep the introduction as brief as possible, supplementing it by a summary of the main results in section 2 . In section 3 various preliminary results are presented that will be subsequently used. In view of the intricacies mentioned above, we chose to write a self-contained paper to facilitate the reading. Therefore, we revisit the Breitenlohner-Maison linear system and the integrability of the field equations in section 4 , and in section 5 we introduce and discuss monodromy matrices. In section 6 we prove the main theorem of the paper, which states that the canonical Wiener-Hopf factorization of a monodromy matrix always yields exact solutions, in both cases $\sigma= \pm 1$. In section 7 we discuss the possibility to obtain such solutions from other types of matrix factorizations, allowing for poles in the factors. In section 8 we present a thorough study of the exact solutions that can be obtained from the canonical factorization, for $\sigma= \pm 1$, of a very simple rational diagonal monodromy matrix, given by (2.19) with $\epsilon=1$. We call this monodromy matrix the Schwarzschild monodromy matrix. We show that by using the different allowed choices of the factorization contour, one can construct a 
wide class of metrics, including for instance the solution that describes the interior region of the Schwarzschild black hole. In section 9 we discuss the canonical factorization of the monodromy matrix (2.19) with $\epsilon=0$. The resulting solutions include the Rindler metric and a cosmological Kasner solution. In section 10 we return to one of the new solutions given in [6], complete its analysis and study new cases. Further details about these solutions are given in appendix A. In appendix B we discuss jumps of the transverse extrinsic curvature that arise in the presence of null hypersurfaces in space-time across which the metric tensor is only continuous. In appendix $\mathrm{C}$ we summarize the class of $A$-metrics, which here is obtained from the canonical factorization of the monodromy matrix (2.19).

\section{Summary of the main results}

To be able to use a Wiener-Hopf factorization to obtain solutions of the field equations of gravitational theories in $D$ space-time dimensions, we consider gravitational theories that satisfy certain requirements, as follows. Firstly, we restrict to gravitational theories in the absence of a cosmological constant, that is, gravitational theories that have Minkowski space-time as a vacuum solution. Secondly, we focus on the subspace of solutions possessing sufficiently many commuting isometries so that the theory can be dimensionally reduced, first to three dimensions, and subsequently to two dimensions. Thirdly, we take the dimensionally reduced theory in three dimensions to be described by a scalar sigma-model coupled to three-dimensional gravity, such that the target space of the sigma-model is a symmetric space $G / H$. This target space is endowed with an involution, associated with an involutive Lie algebra automorphism of the Lie algebra of $G$. This involution, also called 'generalized transposition', leaves the coset representative $M \in G / H$ invariant. For instance, for $H \subset$ $O(n)$, this involution acts by matrix transposition, while for $H \subset U(n)$ it acts by Hermitian conjugation. The involution acts anti-homomorphically on $g \in G$ (see [5] for a review).

The problem of solving the gravitational field equations in $D$ dimensions is then reduced to solving a system of non-linear second order PDE's depending on two coordinates, which we denote by $(\rho, v) \in \mathbb{R}^{2}$, with $\rho>0$ and $v \in \mathbb{R}$. These coordinates are called Weyl coordinates, and accordingly we will denote the $(\rho, v)$ upper-half plane by Weyl upper-half plane. The system of non-linear second order PDE's to be solved is given by $[16,17]$

$$
d(\rho \star A)=0,
$$

where $A$ denotes the matrix one-form

$$
A=M^{-1} d M .
$$

Here, $\star$ denotes the Hodge star operator in two dimensions, which maps a one-form to a one-form, and satisfies

$$
(\star)^{2}=-\sigma \mathrm{id}, \quad \star d \rho=-\sigma d v, \quad \star d v=d \rho .
$$

We recall that $\sigma= \pm 1$ distinguishes between the case where both $\rho$ and $v$ are space-like coordinates $(\sigma=1)$, and the case where one of them is a time-like and the other one is space-like $(\sigma=-1)$. 
Given a solution of (2.1), one then obtains a solution to the gravitational field equations. Note that, if $M$ is a diagonal matrix, $M^{-1}$ also provides a solution to the field equations. As an illustration, let us consider solutions to the field equations of General Relativity in four dimensions that possess two commuting isometries. Accordingly, we take the space-time metric to have the Weyl-Lewis-Papapetrou form

$$
d s_{4}^{2}=-\sigma \Delta(d y+B d \phi)^{2}+\Delta^{-1}\left(e^{\psi} d s_{2}^{2}+\rho^{2} d \phi^{2}\right)
$$

with $\Delta>0$, where $d s_{2}^{2}$ denotes a flat, two-dimensional line element, which we take to be either

$$
d s_{2}^{2}=\sigma d \rho^{2}+d v^{2}
$$

or

$$
d s_{2}^{2}=d \rho^{2}+\sigma d v^{2} .
$$

$\Delta, B$ and $\psi$ are functions of the Weyl coordinates $(\rho, v)$ only. By dimensionally reducing over $(y, \phi)$ to two dimensions, the functions $\Delta$ and $B$ become encoded in the matrix $M$, which in this case is a two-by-two matrix. Thus, given a solution $M$ of (2.1), one immediately reads off the expressions for $\Delta$ and $B$. The function $\psi$ in (2.4) is determined by integration [16, 17],

$$
\partial_{\rho} \psi=\frac{1}{4} \rho \operatorname{Tr}\left(A_{\rho}^{2}-\sigma A_{v}^{2}\right), \quad \partial_{v} \psi=\frac{1}{2} \rho \operatorname{Tr}\left(A_{\rho} A_{v}\right) .
$$

Therefore, the central question is: how do we determine a solution $M(\rho, v)$ of $(2.1)$ ?

To do so, we proceed in three steps.

Step 1. We use the fact that the non-linear PDE's (2.1) form an integrable system, i.e. they are the solvability conditions [18] for a certain Lax pair, the so-called Breitenlohner-Maison (BM) linear system [2]. This is an auxiliary linear system in two dimensions given by

$$
\varphi(\rho, v)[d X(\rho, v)+A(\rho, v) X(\rho, v)]=\star d X(\rho, v),
$$

specified in terms of the matrix one-form $A=M^{-1} d M$ and a function $\varphi$ of the form

$$
\varphi(\rho, v)=\frac{-\sigma(\omega-v) \pm \sqrt{(\omega-v)^{2}+\sigma \rho^{2}}}{\rho}, \text { with } \omega \in \mathbb{C} \backslash\left\{v_{0} \pm \sqrt{-\sigma} \rho_{0}\right\}
$$

where $\left(\rho_{0}, v_{0}\right)$ is a point in the neighbourhood of which the linear system is to be solved. We denote by $\mathcal{T}$ the set of all functions of this form. If the BM linear system has a non-trivial solution satisfying certain invertibility and differentiability conditions, then $A=M^{-1} d M$ satisfies (2.1).

Note that $\pm \sqrt{-\sigma}$ are the fixed points of the involution $\iota_{\sigma}$ in $\mathbb{C} \backslash\{0\}$,

$$
\iota_{\sigma}(\tau)=-\frac{\sigma}{\tau},
$$

which will play a fundamental role. 
Step 2. We will assume that we can define an involutive map $\sharp$ acting on matrix functions $N(\tau, \rho, v)$ which coincides with the 'generalized transposition' in $G / H$ whenever $N \in G / H$. Examples thereof are $N^{\natural}(\tau, \rho, v)=N^{T}(\tau, \rho, v)$ and $N^{\natural}(\tau, \rho, v)=\eta N^{\dagger}(\bar{\tau}, \rho, v) \eta^{-1}$, where in the second case $\eta$ denotes a constant invertible matrix and $\tau$ takes values in the unit disc. We also assume that $\mathcal{T}$ is closed under $\bigsqcup$.

If we take $\varphi, \chi=-\sigma / \varphi^{\natural} \in \mathcal{T}$, and if $X$ and $\tilde{X}$ denote solutions to the corresponding $\mathrm{BM}$ linear system, then we can prove that the matrix

$$
\mathcal{M}(\rho, v)=\tilde{X}^{\natural}(\rho, v) M(\rho, v) X(\rho, v)
$$

satisfies

$$
d \mathcal{M}(\rho, v)=0 .
$$

Thus, the matrix $\mathcal{M}$ is independent of the Weyl coordinates, even though all the individual factors depend on $(\rho, v)$. The expression (2.11) shows that $\mathcal{M}$ can be constructed by a product involving solutions to the BM linear system and a matrix $M$ that solves the field equations (2.1).

Step 3. Now we turn to the reverse question: given a matrix $\mathcal{M}$ that is independent of the Weyl coordinates, can we construct a factorization of the form (2.11), such that the middle factor $M(\rho, v)$ solves the field equations, and the factor $X$ provides a solution to the BM linear system? If this can be done, we say that $\mathcal{M}$ is a monodromy matrix for $M(\rho, v)$.

This is where Wiener-Hopf factorization comes into play. We will have to make certain (though very general) assumptions, that we summarize as follows (these assumptions may seem a bit technical, but in fact they are natural conditions in order for the class of involutions $\downarrow$ to be as general as possible).

Let $\mathcal{M}(u)$ be an invertible matrix function of the complex variable $u$, and consider the matrix that is obtained by composition with

$$
u=v+\sigma \frac{\rho}{2} \frac{\sigma-\tau^{2}}{\tau}, \quad \tau \in \mathbb{C} \backslash\{0\},
$$

i.e.

$$
\mathcal{M}\left(v+\sigma \frac{\rho}{2} \frac{\sigma-\tau^{2}}{\tau}\right), \quad \tau \in \mathbb{C} \backslash\{0\} .
$$

For each $(\rho, v)$, the relation (2.13) is an algebraic curve in the complex variables $u$ and $\tau$, called the spectral curve. It plays a fundamental role in this study. Note also that if we replace $\tau$ by any $\varphi \in \mathcal{T}$, the relation (2.13) is satisfied for $u=\omega$.

In (2.14), we consider $(\rho, v)$ as an arbitrary pair of parameters in a neighbourhood of $\left(\rho_{0}, v_{0}\right)$, and take $\tau$ as the (complex) independent variable. To emphasize this aspect, we will denote the matrix $\mathcal{M}$ in $(2.14)$ by $\mathcal{M}_{(\rho, v)}(\tau)$.

Assumption 1. There exists an open set $S$ in the Weyl upper-half plane such that, for every $\left(\rho_{0}, v_{0}\right) \in S$, one can find a simple closed curve $\Gamma$ in the $\tau$-plane, which is $\iota_{\sigma}$-invariant and encircles the origin, such that: for all $(\rho, v)$ in a neighbourhood of $\left(\rho_{0}, v_{0}\right), \mathcal{M}_{(\rho, v)}^{ \pm 1}(\tau)$ is analytic in an $\iota_{\sigma}$-invariant open set $O$ in the $\tau$-plane, containing $\Gamma$ (see figure 1$)$, and such that $\mathcal{M}_{(\rho, v)}^{\natural}(\tau)=\mathcal{M}_{(\rho, v)}(\tau)$ on $O$. 
Let $D^{+}$denote the simply connected interior region of $\Gamma$, and $D^{-}=\mathbb{C} \backslash\left(D^{+} \cup \Gamma\right)$.

Assumption 2. If $N(\tau, \rho, v)$ is analytic in $D^{+} \cup O$, then $N^{\natural}(\tau, \rho, v)$ is also analytic in $D^{+} \cup O$.

Assumption 3. $\mathcal{M}_{(\rho, v)}(\tau)$ admits a canonical Wiener-Hopf factorization with respect to $\Gamma$,

$$
\mathcal{M}_{(\rho, v)}(\tau)=M_{(\rho, v)}^{-}(\tau) M_{(\rho, v)}^{+}(\tau) \text { on } \Gamma
$$

where $M_{(\rho, v)}^{+}(\tau)$ (respectively $M_{(\rho, v)}^{-}(\tau)$ ) and its inverse are analytic and bounded in the open set $D^{+} \cup O$ (respectively $\left.D^{-} \cup O\right)$. $M_{(\rho, v)}^{+}(\tau)$ satisfies the normalization condition $M_{(\rho, v)}^{+}(0)=\mathbb{I}$. We set $X(\tau, \rho, v)=M_{(\rho, v)}^{+}(\tau)$, so $X(0, \rho, v)=\mathbb{I}$.

Consider an $n \times n$ matrix function $\mathcal{M}(\tau), \tau \in \Gamma$, such that both $\mathcal{M}$ and $\mathcal{M}^{-1}$ are continuous on $\Gamma$. A representation of $\mathcal{M}(\tau), \tau \in \Gamma$, as a product

$$
\mathcal{M}(\tau)=M_{-}(\tau) d(\tau) M_{+}(\tau)
$$

where $d$ is a diagonal matrix of the form $d(\tau)=\operatorname{diag}\left(\tau^{k_{j}}\right)_{j=1,2, \ldots, n}$ with $k_{j} \in \mathbb{Z}$, and where $M_{+}^{ \pm 1}$ (respectively $M_{-}^{ \pm 1}$ ) admit bounded analytic extensions to $D^{+}$(respectively $D^{-}$), is called a (bounded) Wiener-Hopf factorization. When $d=\mathbb{I}$, this factorization is called canonical. The latter, if it exists, is unique, up to a constant matrix factor which can be fixed by imposing a normalization condition, such as $M_{+}(0)=\mathbb{I}[10,19,20]$. Then, as shown in [5], (2.15) can be written as

$$
\mathcal{M}_{(\rho, v)}(\tau)=X^{\natural}\left(-\frac{\sigma}{\tau}, \rho, v\right) M(\rho, v) X(\tau, \rho, v), \quad \tau \in \Gamma,
$$

where

$$
M(\rho, v)=\lim _{\tau \rightarrow \infty} M_{(\rho, v)}^{-}(\tau)=M_{(\rho, v)}^{-}(\infty) .
$$

Assumption 4. $M(\rho, v)$ is of class $C^{2}$, and for each $\tau \in D^{+} \cup O$ the matrix function $X$ is of class $C^{2}$ as a function of $(\rho, v)$, and $\partial X / \partial \rho, \partial X / \partial v, \partial X^{\natural} / \partial \rho$ and $\partial X^{\natural} / \partial v$ are analytic as functions of $\tau$ in the domain $D^{+} \cup O$.

Under these assumptions, we have the following main theorem:

Theorem 1. Let Assumptions 1-4 be satisfied. Then $M(\rho, v)$ is a solution of the field equations (2.1) on $S$.

The proof of this theorem gives, moreover, an affirmative answer to the question raised in Step 3.

By using this result, we study the solutions to the field equations of General Relativity in four dimensions that arise from the canonical Wiener-Hopf factorization of a particular type of matrices. These monodromy matrices are chosen to be of the simplest possible, non-constant kind, namely, they are diagonal, rational, with $\operatorname{det} \mathcal{M}=1$, possessing only one zero and one pole in the extended $u$-plane, as follows,

$$
\mathcal{M}(u)=\left[\begin{array}{cc}
\sigma \frac{u-\epsilon m}{\epsilon u+m} & 0 \\
0 & \sigma \frac{\epsilon u+m}{u-\epsilon m}
\end{array}\right], \quad m \in \mathbb{R}^{+}, \quad \epsilon=0,1 .
$$


Note that the matrix $\mathcal{M}_{(\rho, v)}(\tau)$, obtained from (2.19) by composition with (2.13), always admits a canonical Wiener-Hopf factorization [6]. From that factorization we obtain, by choosing different possible factorization contours, a wide class of solutions that includes all type $A$ space-time metrics, a cosmological Kasner solution and the Rindler metric, as well as solutions whose metric tensor is continuous but not smooth due to the presence of null hypersurfaces $[7,8,12,13]$.

Although the matrices $\mathcal{M}_{(\rho, v)}(\tau)$ in the cases $\sigma=1$ and $\sigma=-1$ are very similar, the behaviour of the corresponding factors $M(\rho, v)$ is remarkably different, due to the presence of square roots that may vanish along certain lines in the $(\rho, v)$ upper-half plane when $\sigma=-1$. Such lines are absent when $\sigma=1$. Obtaining the $A$-metrics for $\sigma=-1$ also involves extending the real-valued solutions by affine transformations in the $(\rho, v)$ upperhalf plane, followed by appropriate changes of coordinates.

So far, our discussion focussed on canonical factorizations of monodromy matrices. What about other types of factorizations? Do other types of matrix factorizations, where different analyticity and normalization conditions are imposed on the factors (allowing them to be meromorphic, for instance), also yield solutions to the field equations, at least in certain cases? To address this question, we consider the example of a cosmological Kasner solution which, when expressed in terms of Weyl coordinates, belongs to the class $\sigma=-1$. By solving the BM linear system for this solution, we construct an infinite set of monodromy matrices. We then pick one of them, and we show that this particular monodromy matrix possesses a meromorphic factorization [21] that gives back the Kasner solution, whereas its canonical factorization gives rise to a different solution to the field equations. Both solutions to the field equations are, however, related by a certain transformation, which we give in section 7. To our knowledge, this is the first time that it is shown that a meromorphic factorization of a monodromy matrix can give rise to a solution of the gravitational field equations.

In section 10 we return to one of the monodromy matrices introduced in [6]. Its canonical factorization can give rise to various solutions due to different choices of the factorization contour. The contour considered in [6] was the unit circle on the complex $\tau$-plane. There is, however, no need to pick this particular contour: as mentioned in Assumption 1, we are free to choose other simple closed contours that enclose the origin and pass through the fixed points of the involution (2.10). By replacing the unit circle with another such contour, we complete the analysis of the solution obtained in [6] and find that is possesses two Killing horizons. We give the exact expression for the space-time metric, and we discuss its interpretation. The solution discussed in [6] carries one electric charge $Q>0$ and one magnetic charge $P>0$. We also discuss what happens when $Q P<0$, for the same choice of the factorization contour. We find that the case $Q P<0$ is markedly different from the case $Q P>0$ discussed in [6].

\section{Preliminary results}

We begin by introducing the involution $\iota_{\sigma}$ in $\mathbb{C} \backslash\{0\}$,

$$
\iota_{\sigma}(\tau)=-\frac{\sigma}{\tau} .
$$


It has two fixed points, which we denote by $\pm p_{F}$ : $\pm i$ if $\sigma=1$, and \pm 1 if $\sigma=-1$. We will denote the set of fixed points by $F P_{\sigma}$.

Next, we introduce the set $W_{\rho, v}=\{v \pm \sqrt{-\sigma} \rho\}$ for any $(\rho, v)$ in the Weyl upper-half plane. We can write this set as $W_{\rho, v}=\left\{v \pm p_{F} \rho\right\}$.

Remark. Let

$$
\omega=v+\sigma \frac{\rho}{2} \frac{\sigma-\tau^{2}}{\tau}, \quad \tau \in \mathbb{C} \backslash\{0\} .
$$

Then $\tau= \pm p_{F}$ iff $\omega \in W_{\rho, v}$, because $p_{F}^{2}=-\sigma$.

\subsection{Properties of $\varphi \in \mathcal{T}$}

Proposition 3.1. Let $\left(\rho_{0}, v_{0}\right)$ be a point in the Weyl upper half-plane. For all $\omega \in$ $\mathbb{C} \backslash W_{\rho_{0}, v_{0}}$, there exists a branch of the square root such that $\varphi$ and $\widetilde{\varphi}=-\sigma / \varphi$, given by

$$
\varphi(\rho, v)=\frac{-\sigma(\omega-v)+\sqrt{(\omega-v)^{2}+\sigma \rho^{2}}}{\rho},
$$

are of class $C^{\infty}$ in a neighbourhood of $\left(\rho_{0}, v_{0}\right)$.

Proof. Let $\left(\rho_{0}, v_{0}\right)$ be any point in the Weyl upper half-plane. We start by noting that $\left(\omega-v_{0}\right)^{2}+\sigma \rho_{0}^{2}$ vanishes precisely for $\omega \in W_{\rho_{0}, v_{0}}$. So let $\omega \in \mathbb{C} \backslash W_{\rho_{0}, v_{0}}$. Decomposing $\omega=\omega_{R}+i \omega_{I}$ with $\omega_{R}, \omega_{I} \in \mathbb{R}$, we have

$$
\left(\omega-v_{0}\right)^{2}+\sigma \rho_{0}^{2}=\left(\omega_{R}-v_{0}\right)^{2}-\omega_{I}^{2}+\sigma \rho_{0}^{2}+2 i \omega_{I}\left(\omega_{R}-v_{0}\right)
$$

Let us first consider the case $\sigma=1$. We choose the principal branch of $\sqrt{z}$ (i.e. $\arg z \in$ ] $-\pi, \pi]$ ), with branch cut $\mathbb{R}^{-}$. We have $\left(\omega-v_{0}\right)^{2}+\sigma \rho_{0}^{2} \in \mathbb{R}$ iff $\omega_{I}=0$ or $\omega_{R}=v_{0}$. If $\omega_{I}=0$, the real part of (3.4) is positive. If $\omega_{R}=v_{0}$, the real part of (3.4) is also positive unless $\left|\omega_{I}\right|>\rho_{0}$ (we exclude $v_{0} \pm i \rho_{0} \in W_{\rho_{0}, v_{0}}$ from our considerations). Therefore, with the chosen branch of $\sqrt{z}, \sqrt{(\omega-v)^{2}+\rho^{2}}$ is $C^{\infty}$ in a neighbourhood of $\left(\rho_{0}, v_{0}\right)$ unless $\omega_{R}=v_{0}$ and $\left|\omega_{I}\right|>\rho_{0}$. Since in the latter case the real part of (3.4) is negative, we choose the branch of $\sqrt{z}$ with $\arg z \in[0,2 \pi[$ for this case.

When $\sigma=-1$, a similar reasoning leads to the choice of the latter branch for $\sqrt{z}$, unless $\omega_{I}=0$ and $\left|\omega_{R}-v_{0}\right|>\rho_{0}$, in which case we choose the principal branch.

Next, for any given $\left(\rho_{0}, v_{0}\right)$, we define the set $\mathcal{T}_{\rho_{0}, v_{0}}$ of all functions $\varphi$ on the Weyl upper half-plane, of the form

$$
\varphi(\rho, v)=\frac{-\sigma(\omega-v) \pm \sqrt{(\omega-v)^{2}+\sigma \rho^{2}}}{\rho}
$$

for some $\omega \in \mathbb{C} \backslash W_{\rho_{0}, v_{0}}$. Note that if $\varphi \in \mathcal{T}_{\rho_{0}, v_{0}}$, then also $\widetilde{\varphi} \in \mathcal{T}_{\rho_{0}, v_{0}}$, where

$$
\widetilde{\varphi}=-\sigma / \varphi,
$$


i.e. $\mathcal{T}_{\rho_{0}, v_{0}}$ is $\iota_{\sigma}$-invariant. Moreover, for $\varphi \in \mathcal{T}_{\rho_{0}, v_{0}}$, we have that $\varphi\left(\rho_{0}, v_{0}\right) \neq 0$ and $\varphi^{2}\left(\rho_{0}, v_{0}\right)+\sigma \neq 0$. Therefore, $\varphi$ and $\varphi^{2}+\sigma$ do not vanish in a neighbourhood of $\left(\rho_{0}, v_{0}\right)$. Also note that for any $\varphi \in \mathcal{T}_{\rho_{0}, v_{0}}$, that is $\varphi$ of the form (3.5), we have

$$
v+\sigma \frac{\rho}{2}\left(\frac{\sigma-\varphi^{2}(\rho, v)}{\varphi(\rho, v)}\right)=\omega .
$$

This relation is invariant under the replacement of $\varphi$ by $\widetilde{\varphi}$.

Now we discuss various properties of $d \varphi$ which are valid in a neighbourhood of $\left(\rho_{0}, v_{0}\right)$ where $\varphi \in \mathcal{T}_{\rho_{0}, v_{0}}$ is of class $C^{\infty}$. For ease of notation, we will from now on denote $\mathcal{T}_{\rho_{0}, v_{0}}$ simply by $\mathcal{T}$.

Proposition 3.2. Let $\varphi \in \mathcal{T}$. Then we have

$$
\begin{aligned}
d \varphi & =\frac{\varphi}{\rho}\left[\frac{\sigma-\varphi^{2}}{\sigma+\varphi^{2}} d \rho+\frac{2 \sigma \varphi}{\sigma+\varphi^{2}} d v\right], \\
\star d \varphi & =\sigma \frac{\varphi}{\rho}\left[-\frac{\sigma-\varphi^{2}}{\sigma+\varphi^{2}} d v+\frac{2 \varphi}{\sigma+\varphi^{2}} d \rho\right], \\
\frac{2 \varphi \sigma}{\varphi^{2}+\sigma} d \varphi+\frac{\varphi^{2}-\sigma}{\varphi^{2}+\sigma} \star d \varphi & =\sigma \frac{\varphi}{\rho} d v .
\end{aligned}
$$

Proof. Recall that $\varphi \neq 0$ and $\varphi^{2}+\sigma \neq 0$ for $\varphi \in \mathcal{T}$. Equation (3.8) is easily verified, and equation (3.9) follows by using (2.3). Then,

$$
\begin{aligned}
\frac{2 \varphi \sigma}{\varphi^{2}+\sigma} d \varphi+\frac{\varphi^{2}-\sigma}{\varphi^{2}+\sigma} \star d \varphi= & \frac{1}{\left(\varphi^{2}+\sigma\right)^{2}} \frac{\varphi}{\rho}\left[2 \sigma \varphi\left(\sigma-\varphi^{2}\right) d \rho+4 \varphi^{2} \sigma^{2} d v+\sigma\left(\varphi^{2}-\sigma\right)^{2} d v\right. \\
& \left.+\left(\varphi^{2}-\sigma\right) 2 \sigma \varphi d \rho\right] \\
= & \frac{1}{\left(\varphi^{2}+\sigma\right)^{2}} \frac{\varphi}{\rho} \sigma\left(\varphi^{2}+\sigma\right)^{2} d v=\sigma \frac{\varphi}{\rho} d v,
\end{aligned}
$$

which yields (3.10).

Proposition 3.3. Let $\varphi \in \mathcal{T}$. Then

$$
d\left(\rho \star \frac{d \varphi}{\varphi}\right)=0 .
$$

Proof. Recall that $\varphi \neq 0$ and $\varphi^{2}+\sigma \neq 0$ for $\varphi \in \mathcal{T}$. We set

$$
S=\frac{2 \varphi}{\sigma+\varphi^{2}}, \quad C=\frac{\sigma-\varphi^{2}}{\sigma+\varphi^{2}},
$$

and compute

$$
d S=2 C \frac{d \varphi}{\sigma+\varphi^{2}}, \quad d C=-\sigma 2 S \frac{d \varphi}{\sigma+\varphi^{2}} .
$$

Using (3.8) in the form

$$
d \varphi=\frac{\varphi}{\rho}(C d \rho+\sigma S d v)
$$

we obtain

$$
d\left(\rho \star \frac{d \varphi}{\varphi}\right)=\sigma \frac{2}{\sigma+\varphi^{2}}(\sigma S d \varphi \wedge d v+C d \varphi \wedge d \rho)=\sigma \frac{2 \rho}{\varphi\left(\sigma+\varphi^{2}\right)} d \varphi \wedge d \varphi=0 .
$$




\subsection{Contour properties}

The following result will be needed in subsequent sections.

Proposition 3.4. Let $\Gamma$ be a simple closed curve in the complex $\tau$-plane, such that it encircles the origin of the $\tau$-plane, and such that it is invariant under the involution $\iota_{\sigma}$. Let $D^{+}$denote the simply connected interior region of $\Gamma$ (i.e. $0 \in D^{+}$), and let $D^{-}=$ $\mathbb{C} \backslash\left(D^{+} \cup \Gamma\right)$, so that $D^{+} \cup \Gamma \cup D^{-}=\mathbb{C}$. Let $p \in \mathbb{C} \backslash\{0\}$. If $p \in D^{+}$then $\iota_{\sigma}(p) \in D^{-}$, and if $p \in D^{-}$then $\iota_{\sigma}(p) \in D^{+}$.

Proof. We take $\Gamma$ to be positively oriented. Consider a point $w_{0} \in \mathbb{C} \backslash(\{0\} \cup \Gamma)$. Then, the winding number of $\Gamma$ around $w_{0}$ is either zero or one. Now, for any such $w_{0}$,

$$
\begin{aligned}
\frac{1}{2 \pi i} \oint_{\Gamma} \frac{d z}{z-w_{0}} & =\frac{1}{2 \pi i} \frac{\sigma}{w_{0}} \oint_{\Gamma} \frac{d w}{\left(w+\frac{\sigma}{w_{0}}\right) w}=\frac{1}{2 \pi i} \oint_{\Gamma} \frac{d w}{w}-\frac{1}{2 \pi i} \oint_{\Gamma} \frac{d w}{w+\frac{\sigma}{w_{0}}} \\
& =1-\frac{1}{2 \pi i} \oint_{\Gamma} \frac{d w}{w+\frac{\sigma}{w_{0}}},
\end{aligned}
$$

where we used the invariance of $\Gamma$ under $\iota_{\sigma}$. Hence, if the winding number around $w_{0}$ is zero, the winding number around $\iota_{\sigma}\left(w_{0}\right)$ is one, and vice-versa.

Corollary 3.4.1. Let $\Gamma$ be a simple closed curve in the complex $\tau$-plane, such that it encircles the origin of the $\tau$-plane, and such that it is invariant under the involution $\iota_{\sigma}$. Then, $\Gamma$ passes through the fixed points of $\iota_{\sigma}$.

Proof. This is a simple consequence of Proposition 3.4. Let $w_{0}$ denote one of the fixed points. If $w_{0} \notin \Gamma$, then either $w_{0} \in D^{+}$or $w_{0} \in D^{-}$, in which case $w_{0}$ and $\iota_{\sigma}\left(w_{0}\right)$ have different winding numbers by Proposition 3.4, which contradicts the assumption that $w_{0}$ is a fixed point of $\iota_{\sigma}$ (i.e. $w_{0}=\iota_{\sigma}\left(w_{0}\right)$ ).

\subsection{Affine transformations}

We close this section with a discussion of changes of Weyl coordinates $(\rho, v) \mapsto(\tilde{\rho}, \tilde{v})$ that preserve the form of the two-dimensional line element $d s_{2}^{2}$, given either by (2.5) or by (2.6). Namely, we consider affine transformations

$$
\left(\begin{array}{l}
\tilde{\rho} \\
\tilde{v}
\end{array}\right)=\left(\begin{array}{ll}
a & b \\
c & d
\end{array}\right)\left(\begin{array}{l}
\rho \\
v
\end{array}\right)+\left(\begin{array}{l}
\alpha \\
\beta
\end{array}\right), \quad a, b, c, d, \alpha, \beta \in \mathbb{R} .
$$

Invertibility of the linear part of the transformation requires $a d-b c \neq 0$. In addition, we demand that $\sigma d \rho^{2}+d v^{2}=k\left(\sigma d \tilde{\rho}^{2}+d \tilde{v}^{2}\right)$, where $k \in \mathbb{R} \backslash\{0\}$. This requires imposing

$$
\sigma a^{2}+b^{2}=\sigma d^{2}+c^{2}, \quad a c=-\sigma b d,
$$

or equivalently,

$$
\sigma a^{2}+c^{2}=\sigma d^{2}+b^{2}, \quad a b=-\sigma c d .
$$


Thus, the linear part of the transformation is a linear conformal isometry when $k>0$, and a linear conformal anti-isometry when $k<0$.

Under the change of Weyl coordinates $(\tilde{\rho}, \tilde{v}) \mapsto(\rho, v)$, a solution $\tilde{M}(\tilde{\rho}, \tilde{v})$ to $(2.1)$ gets mapped to $\tilde{M}(\tilde{\rho}(\rho, v), \tilde{v}(\rho, v)) \equiv M(\rho, v)$, which satisfies the field equations

$$
d\left(\tilde{\rho}(\rho, v) M^{-1}(\rho, v) \star d M(\rho, v)\right)=0 .
$$

\section{The Breitenlohner-Maison linear system}

The non-linear equations (2.1) are an integrable system, i.e., they are the solvability conditions [18] for a certain Lax pair, the so-called Breitenlohner-Maison (BM) linear system [2]. This is an auxiliary linear system in the Weyl upper-half plane given by

$$
\varphi(\rho, v)(d X(\rho, v)+A(\rho, v) X(\rho, v))=\star d X(\rho, v) .
$$

It is defined in terms of a function $\varphi$ and a matrix one-form $A$. The latter takes the form given in (2.2). We assume that the components of $A$ are one-forms, whose coefficient functions are continuously differentiable functions in an open set in the Weyl upper-half plane. We will refer to this requirement by saying that $A$ is of class $C^{1}$. The function $\varphi$ is taken from the set $\mathcal{T}$ defined in (3.5).

Given $A$ and $\varphi$, we seek solutions $X$ with the following properties: the matrix $X$ is invertible; $X$ is twice continuously differentiable (i.e. of class $C^{2}$ ) and $X^{-1}$ is continuously differentiable (i.e. of class $\left.C^{1}\right)$ with respect to $(\rho, v)$.

Next, let us discuss the solvability of the BM linear system (4.1), following [5, 17]. We will make use of the relations

$$
d\left(d X X^{-1}\right)=d X X^{-1} \wedge d X X^{-1}
$$

and

$$
d(\star A)=\frac{1}{\rho} d(\rho \star A)-\frac{1}{\rho} d \rho \wedge \star A,
$$

where we recall that $\rho>0$.

Proposition 4.1. Let $\varphi \in \mathcal{T}$. Then

$$
\begin{aligned}
\varphi(d X+A X) & =\star d X \\
& \Longleftrightarrow \\
\left(\varphi^{2}+\sigma\right) d X X^{-1} & =-\varphi^{2} A-\varphi \star A .
\end{aligned}
$$

Proof. $\Rightarrow$ : we multiply (4.1) by $X^{-1}$ on the right, to obtain

$$
\varphi d X X^{-1}+\varphi A=\star d X X^{-1} .
$$

Using (2.3), we obtain for its Hodge dual,

$$
\varphi \star d X X^{-1}+\varphi \star A=-\sigma d X X^{-1} .
$$


Multiplying by $\varphi$ gives

$$
\left\{\begin{array}{l}
\varphi^{2} d X X^{-1}+\varphi^{2} A-\varphi \star d X X^{-1}=0 \\
\varphi \star d X X^{-1}+\varphi \star A+\sigma d X X^{-1}=0 .
\end{array}\right.
$$

Their sum simplifies to

$$
\left(\varphi^{2}+\sigma\right) d X X^{-1}=-\varphi^{2} A-\varphi \star A .
$$

$\Leftarrow$ : recall that for $\varphi \in \mathcal{T}$ we have $\varphi^{2}+\sigma \neq 0$. Starting with

$$
\left(\varphi^{2}+\sigma\right) d X X^{-1}=-\varphi^{2} A-\varphi \star A,
$$

and multiplying by $X$ on the right gives,

$$
\left(\varphi^{2}+\sigma\right) d X=-\varphi^{2} A X-\varphi \star A X .
$$

Applying the Hodge star operator to it, and multiplying it by $\varphi$, respectively, we obtain the following equations,

$$
\left\{\begin{array}{l}
\varphi^{2} \star d X+\sigma \star d X=-\varphi^{2} \star A X+\varphi \sigma A X, \\
\varphi^{3} d X+\varphi \sigma d X=-\varphi^{3} A X-\varphi^{2} \star A X .
\end{array}\right.
$$

Subtracting the second equation from the first, we get

$$
\left(\varphi^{2}+\sigma\right) \star d X-\left(\varphi^{2}+\sigma\right) \varphi d X=\left(\varphi^{2}+\sigma\right) \varphi A X
$$

which, when divided by $\left(\varphi^{2}+\sigma\right)$, gives the BM linear system

$$
\varphi(d X+A X)=\star d X .
$$

Now we show that the solvability of the linear system (4.1) implies the field equations (2.1).

Theorem 4.2. Let $\varphi \in \mathcal{T}$. If the equation for the BM linear system,

$$
\varphi(d X+A X)=\star d X
$$

is satisfied, then $A$ is a solution to the field equations

$$
d(\rho \star A)=0 .
$$

Proof. Using Proposition 4.1, and taking the differential of (4.4), we get

$$
2 \varphi d \varphi \wedge d X X^{-1}+\left(\varphi^{2}+\sigma\right) d\left(d X X^{-1}\right)=-2 \varphi d \varphi \wedge A-\varphi^{2} d A-d \varphi \wedge \star A-\varphi d(\star A) .
$$

By (4.2) and (4.3), the previous equation becomes

$$
\begin{aligned}
2 \varphi d \varphi \wedge d X X^{-1}+\left(\varphi^{2}+\sigma\right) d X X^{-1} \wedge d X X^{-1}= & -2 \varphi d \varphi \wedge A-\varphi^{2} d A-d \varphi \wedge \star A-\frac{\varphi}{\rho} d(\rho \star A) \\
& -\frac{\varphi}{\rho} d \varphi \wedge \star A
\end{aligned}
$$


Substituting (4.4) into it, we obtain

$$
\begin{aligned}
&-\frac{\varphi}{\rho} d \varphi \wedge \star A= 2 \varphi d \varphi \wedge\left(-\frac{\varphi^{2}}{\varphi^{2}+\sigma} A-\frac{\varphi}{\varphi^{2}+\sigma} \star A\right) \\
&+\left(\varphi^{2}+\sigma\right)\left[\frac{\varphi^{4}}{\left(\varphi^{2}+\sigma\right)^{2}} A \wedge A+\frac{\varphi^{3}}{\left(\varphi^{2}+\sigma\right)^{2}} A \wedge \star A\right. \\
&\left.+\frac{\varphi^{3}}{\left(\varphi^{2}+\sigma\right)^{2}} \star A \wedge A+\frac{\varphi^{2}}{\left(\varphi^{2}+\sigma\right)^{2}} \star A \wedge \star A\right] \\
&+2 \varphi d \varphi \wedge A+\varphi^{2} d A+d \varphi \wedge \star A-\frac{\varphi}{\rho} d \varphi \wedge \star A .
\end{aligned}
$$

Now we use the following relations for one-forms $B, C$, valid in two dimensions,

$$
\left\{\begin{aligned}
\star B \wedge \star C & =\sigma B \wedge C, \\
\star B \wedge C & =-B \wedge \star C,
\end{aligned}\right.
$$

as well as the relation $d A+A \wedge A=0$ satisfied by $A=M^{-1} d M$. These relations, together with (3.8), lead to a simplification of (4.18),

$$
-\frac{\varphi}{\rho} d(\rho \star A)=-\frac{2 \varphi^{3}}{\varphi^{2}+\sigma} d \varphi \wedge A-\frac{2 \varphi^{2}}{\varphi^{2}+\sigma} d \varphi \wedge \star A+2 \varphi d \varphi \wedge A+d \varphi \wedge A-\frac{\varphi}{\rho} d \rho \wedge \star A .
$$

This can be written as

$$
-\frac{\varphi}{\rho} d(\rho \star A)=\frac{2 \varphi \sigma}{\varphi^{2}+\sigma} d \varphi \wedge A+\left(\frac{2 \varphi^{2}}{\varphi^{2}+\sigma}-1\right) \star d \varphi \wedge A+\frac{\varphi}{\rho} \star d \rho \wedge A .
$$

Then, using (2.3), this results in

$$
-\frac{\varphi}{\rho} d(\rho \star A)=\left[\frac{2 \varphi \sigma}{\varphi^{2}+\sigma} d \varphi+\left(\frac{\varphi^{2}-\sigma}{\varphi^{2}+\sigma}\right) \star d \varphi-\sigma \frac{\varphi}{\rho} d v\right] \wedge A .
$$

Using (3.10) and $\varphi \neq 0$, we obtain

$$
d(\rho \star A)=0 .
$$

\section{Monodromy matrix}

The BM linear system (4.1) uses, as an input, a matrix one-form $A=M^{-1} d M$ and a function $\varphi \in \mathcal{T}$. These quantities are defined on the Weyl upper half-plane. Given a solution $X(\rho, v)$ to the BM linear system, Breitenlohner and Maison constructed [2] a matrix $\mathcal{M}$ that is independent of the Weyl coordinates $(\rho, v)$. Here we revisit and generalize their construction. In doing so, we refrain from explicitly indicating the dependence of $\varphi \in \mathcal{T}$ on the Weyl coordinates $(\rho, v)$, for ease of notation.

We will assume that we can define an involutive map $\downarrow$ acting on matrix functions $N(\rho, v)$ of a given order such that

$$
\left(N_{1} N_{2}\right)^{\natural}=N_{2}^{\natural} N_{1}^{\natural}, \quad\left(N_{1}+N_{2}\right)^{\natural}=N_{1}^{\natural}+N_{2}^{\natural}, \quad(a N)^{\natural}=a N^{\natural} \quad \forall a \in \mathbb{R},
$$


and whenever $N \in G / H$, it coincides with the 'generalized transposition' in $G / H$. We will assume that this involution commutes with differentiation,

$$
\partial_{\rho}\left(N^{\natural}\right)=\left(\partial_{\rho} N\right)^{\natural}, \quad \partial_{v}\left(N^{\natural}\right)=\left(\partial_{v} N\right)^{\natural},
$$

and that $\mathcal{T}$ is closed under $\natural$. Examples are $N^{\natural}=N^{T}, N^{\natural}=\eta N^{\dagger} \eta^{-1}$, where $\eta$ denotes a constant invertible matrix.

We follow the notation used in [5]. The coset representative $M \in G / H$ takes the form $M=V^{\natural} V$. Since we take $A$ to be of class $C^{1}$ in an open set, $V$ is of class $C^{2}$ in this set. Since $M$ is locally invertible, so is $V$. We decompose $d V V^{-1}$ into one-forms that under the involution $\downarrow$ are either invariant or anti-invariant,

$$
d V V^{-1}=P+Q,
$$

where

$$
P^{\natural}=P, \quad Q^{\natural}=-Q .
$$

Using (5.2), we note the relation

$$
2 P=V A V^{-1}
$$

Given a solution $X$ to the BM linear system with input $(A, \varphi)$, we define [2]

$$
\mathcal{P}=V X .
$$

Now, instead of $\varphi \in \mathcal{T}$, consider picking $\chi \in \mathcal{T}$ with $\chi=-\sigma / \varphi^{\natural}$. Given any solution $\widetilde{X}$ to BM linear system with input $(A, \chi)$, let $\widetilde{\mathcal{P}}$ denote

$$
\widetilde{\mathcal{P}}=V \widetilde{X} .
$$

We then use $\mathcal{P}$ and $\tilde{\mathcal{P}}$ to define the matrix $\mathcal{M}$ by

$$
\mathcal{M}=\widetilde{\mathcal{P}}^{\natural} \mathcal{P}=\widetilde{X}^{\natural} M X .
$$

Note that $X$ is not necessarily a priori related to $\tilde{X}$, except for the relation between $\varphi$ and $\chi$. Hence, (5.8) generalizes the definition given in [2].

Theorem 5.1. Let $\varphi, \chi \in \mathcal{T}$, with $\chi=-\sigma / \varphi^{\natural}$. Let $X$ and $\widetilde{X}$ denote solutions to the corresponding BM linear system based on $A=M^{-1} d M$. Then, the matrix $\mathcal{M}(\rho, v)$ defined in (5.8) satisfies

$$
d \mathcal{M}(\rho, v)=0 .
$$

Proof. We consider the BM linear system with input $(A, \varphi)$ and solution $X$. Then, by Proposition 4.1, $X, A, \varphi$ satisfy,

$$
d X X^{-1}=-\frac{\varphi^{2}}{\varphi^{2}+\sigma} A-\frac{\varphi}{\varphi^{2}+\sigma} \star A .
$$

Using (5.6), we compute

$$
\begin{aligned}
d \mathcal{P} \mathcal{P}^{-1} & =d V V^{-1}+V d X X^{-1} V^{-1} \\
& =Q+P-\frac{2 \varphi^{2}}{\varphi^{2}+\sigma} P-\frac{2 \varphi}{\varphi^{2}+\sigma} \star P=Q-\frac{\varphi^{2}-\sigma}{\varphi^{2}+\sigma} P-\frac{2 \varphi}{\varphi^{2}+\sigma} \star P,
\end{aligned}
$$

where we used the relation (5.10) as well as (5.4) and (5.5). 
Similarly, for the BM linear system with input $(A, \chi)$ and solution $\widetilde{X}$, we obtain

$$
d \widetilde{\mathcal{P}} \widetilde{\mathcal{P}}^{-1}=Q-\frac{\chi^{2}-\sigma}{\chi^{2}+\sigma} P-\frac{2 \chi}{\chi^{2}+\sigma} \star P .
$$

Next, using (5.8) and the property (5.2), we compute

$$
d \mathcal{M}=d\left(\widetilde{\mathcal{P}}^{\natural}\right) \mathcal{P}+\widetilde{\mathcal{P}}^{\natural} d \mathcal{P}=\widetilde{\mathcal{P}}^{\natural}\left[\left(\widetilde{\mathcal{P}}^{\natural}\right)^{-1}(d \widetilde{\mathcal{P}})^{\natural}+d \mathcal{P} \mathcal{P}^{-1}\right] \mathcal{P},
$$

which, using (5.1), can be written as

$$
d \mathcal{M}=\widetilde{\mathcal{P}}^{\natural}\left[\left(d \widetilde{\mathcal{P}} \widetilde{\mathcal{P}}^{-1}\right)^{\natural}+d \mathcal{P} \mathcal{P}^{-1}\right] \mathcal{P} .
$$

Substituting the expression (5.11) and (5.12) into this gives

$$
d \mathcal{M}=\tilde{\mathcal{P}}^{\natural}\left[Q^{\natural}-\left(\frac{\chi^{2}-\sigma}{\chi^{2}+\sigma} P\right)^{\natural}-\left(\frac{2 \chi}{\chi^{2}+\sigma} \star P\right)^{\natural}+Q-\frac{\varphi^{2}-\sigma}{\varphi^{2}+\sigma} P-\frac{2 \varphi}{\varphi^{2}+\sigma} \star P\right] \mathcal{P} .
$$

Then, using (5.4), and taking into account that $\chi^{\natural}=-\sigma / \varphi$, we obtain

$$
d \mathcal{M}=\widetilde{\mathcal{P}}^{\natural}\left[-Q-\frac{-\varphi^{2}+\sigma}{\varphi^{2}+\sigma} P+\frac{2 \varphi}{\varphi^{2}+\sigma} \star P+Q-\frac{\varphi^{2}-\sigma}{\varphi^{2}+\sigma} P-\frac{2 \varphi}{\varphi^{2}+\sigma} \star P\right] \mathcal{P}=0 .
$$

Thus, we have shown that the matrix $\mathcal{M}$ is independent of the Weyl coordinates $(\rho, v)$ for any $X$ and $\widetilde{X}$ that solve (4.1) with input $\varphi$ and $\chi$, respectively.

Thus, the matrix $\mathcal{M}$ defined in (5.8) is independent of the Weyl coordinates, even though the individual factors depend on $(\rho, v)$. This result, which generalizes previous results from (section 3 of) [2] and from (section 5 of) [5], suggests that all information on $M(\rho, v)$ may be lost after multiplication by $X$ and $\widetilde{X}^{\natural}$ on the right and on the left, respectively; indeed we will later give an example in which, for a given $M(\rho, v)$, it is possible to obtain in this way an arbitrary constant matrix $\mathcal{M}$, in particular the identity matrix. In case we have a matrix $\mathcal{M}$, independent of $(\rho, v)$, from which one can obtain, via an appropriate factorization, a solution $M(\rho, v)$ of the field equations, we will follow the terminology in the literature [3] and say that $\mathcal{M}$ is a monodromy matrix for $M(\rho, v)$. This will be addressed in the next section.

\section{Canonical factorization gives a solution to the BM linear system}

In the previous section, we saw that, by (5.8), one can construct matrices $\mathcal{M}$ that are independent of the Weyl coordinates, from given solutions $X$ and $\widetilde{X}$ of the BM linear system with input $(A, \varphi)$ and $(A, \chi)$, respectively, where $\varphi, \chi=-\sigma / \varphi^{\natural} \in \mathcal{T}$. Therefore, we obtain a factorization of $\mathcal{M}$ in terms of $X, \widetilde{X}$ and $M$.

In this section, we study the reverse question. Namely, we obtain $A(\rho, v)$ (or equivalently, $M(\rho, v)$ ) from a factorization of a matrix function $\mathcal{M}$ of the form (6.2) given below. 
Naturally, to do so, we must make certain (though very general) assumptions, as follows (these assumptions may seem a bit technical, but in fact they are natural conditions in order for the class of involutions $\sharp$ to be as general as possible).

Let $\downarrow$ be an involution in the space of matrix functions $N(\tau, \rho, v)$ of a given order, with domain contained in $\mathbb{C} \times \mathbb{R}^{+} \times \mathbb{R}$, satisfying (5.1) and (5.2) and such that it coincides with the 'generalized transposition' if $N(\tau, \rho, v)=N(\rho, v)$ does not depend on $\tau$ and belongs to $G / H$. We assume moreover that $\sharp$ commutes with the involution $\iota_{\sigma}$ and, if $N(\tau, \rho, v)$ and $N^{\natural}(\tau, \rho, v)$ are analytic with respect to $\tau$ in a region $\mathcal{A} \subset \mathbb{C}$, then $\partial_{\tau}\left(N^{\natural}\right)=\left(\partial_{\tau} N\right)^{\natural}$ in this region.

Let $\mathcal{M}(u)$ be a matrix function of the complex variable $u$, and denote by $\mathcal{M}(\tau, \rho, v)$ the matrix that is obtained by composition with

$$
u=v+\sigma \frac{\rho}{2} \frac{\sigma-\tau^{2}}{\tau}, \quad \tau \neq 0
$$

i.e.

$$
\mathcal{M}(\tau, \rho, v)=\mathcal{M}\left(v+\sigma \frac{\rho}{2} \frac{\sigma-\tau^{2}}{\tau}\right)
$$

for all $\tau \in \mathbb{C} \backslash\{0\}, \rho \in \mathbb{R}^{+}, v \in \mathbb{R}$ such that $\mathcal{M}(\tau, \rho, v)$ is invertible.

For later convenience, we note the relation of (6.1), called the spectral curve, with property (3.7). We also recall the definition (3.1) of the involution $\iota_{\sigma}$.

Assumption 1. There exists an open set $S$ such that, for every $\left(\rho_{0}, v_{0}\right) \in S$, one can find a simple closed curve $\Gamma$ in the $\tau$-plane, which is $\iota_{\sigma}$-invariant and encircles the origin, such that: for all $(\rho, v)$ in a neighbourhood of $\left(\rho_{0}, v_{0}\right)$, the matrix (6.2), as well as its inverse, is analytic in a region (i.e. in an open, connected set) in the $\tau$-plane containing $\Gamma$, which we denote by $O$ and which we require to be invariant under $\iota_{\sigma}$ (see figure 1 ), and such that $\mathcal{M}^{\natural}(\tau, \rho, v)=\mathcal{M}(\tau, \rho, v)$ on $O$.

We denote by $D^{+}$the simply connected interior region of $\Gamma$ (hence $0 \in D^{+}$) and by $D^{-}=\mathbb{C} \backslash\left(D^{+} \cup \Gamma\right)$ the exterior region. Recall that with these assumptions, we have, for $p \in \mathbb{C} \backslash\{0\}$, that $p \in D^{+} \Leftrightarrow \iota_{\sigma}(p) \in D^{-}$, see Proposition 3.4, and $\Gamma$ necessarily passes through the fixed points of the involution $\iota_{\sigma}$, which are $\pm i$ when $\sigma=1$ and \pm 1 when $\sigma=-1$, see Corollary 3.4.1.

Assumption 2. If $N(\tau, \rho, v)$ is analytic in $D^{+} \cup O$, then $N^{\natural}(\tau, \rho, v)$ is also analytic in $D^{+} \cup O$.

Now consider $(\rho, v)$ as an arbitrary pair of parameters in a neighbourhood of $\left(\rho_{0}, v_{0}\right)$, and take $\tau$ as the independent (complex) variable. To emphasize this aspect, we will denote the matrix in $(6.2)$ by $\mathcal{M}_{(\rho, v)}(\tau)$.

Assumption 3. With the same notation as in Assumption 1, for any $(\rho, v)$ in a neighbourhood of $\left(\rho_{0}, v_{0}\right), \mathcal{M}_{(\rho, v)}(\tau)$ admits a canonical factorization of Wiener-Hopf (or Birkhoff) type with respect to $\Gamma$,

$$
\mathcal{M}_{(\rho, v)}(\tau)=M_{(\rho, v)}^{-}(\tau) M_{(\rho, v)}^{+}(\tau) \text { on } \Gamma
$$



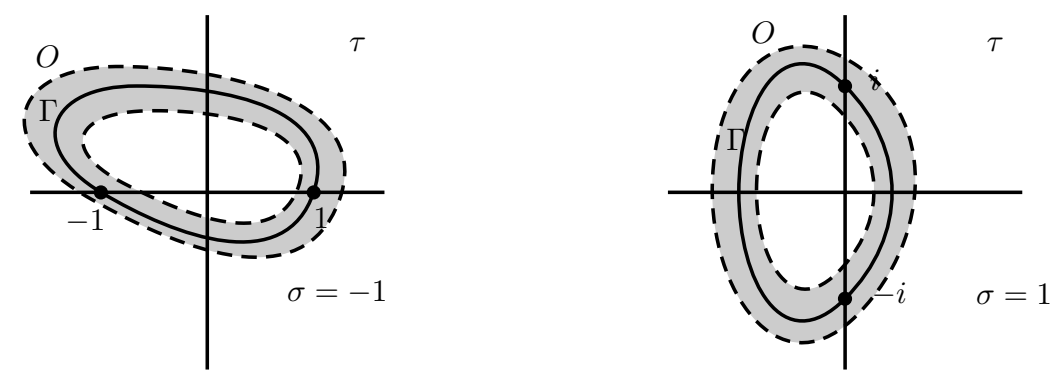

Figure 1. Examples of sets $O$ that are invariant under $\tau \mapsto-\sigma / \tau$. The curve in the middle represents $\Gamma$.

where $M_{(\rho, v)}^{+}(\tau)$ (respectively $M_{(\rho, v)}^{-}(\tau)$ ) and its inverse are analytic and bounded in $D^{+} \cup O$ (respectively $\left.D^{-} \cup O\right)$, and we assume the normalization condition $M_{(\rho, v)}^{+}(0)=\mathbb{I}$. We set $X(\tau, \rho, v)=M_{(\rho, v)}^{+}(\tau)$, so $X(0, \rho, v)=\mathbb{I}$.

Such a factorization, if it exists, is unique; necessary and sufficient conditions for its existence were given in [5] (see also the references therein and [22]). A result given in [6] states that, for any scalar function $f(u)$ that is continuous, as well as its inverse, on $\Gamma_{u}$ (here $\Gamma_{u}$ denotes the image of $\Gamma$ under (6.1)), the function $f_{(\rho, v)}(\tau)$ always admits a canonical factorization with respect to $\Gamma$. It follows from this result that a canonical Wiener-Hopf factorization always exists when the monodromy matrix is triangular [10, 19]. It is also clear that only boundedness of $\mathcal{M}_{(\rho, v)}(\tau)$ in the variable $\tau$, on $\Gamma$, is assumed. $\mathcal{M}(u)$ may be unbounded, see for example section 9. Note that it follows from Assumption 1 that the representation (6.3) holds for all $\tau \in O$.

Moreover, it was shown in [5] that under Assumptions 1 and 2, (6.3) can be written as

$$
\mathcal{M}_{(\rho, v)}(\tau)=X^{\natural}\left(-\frac{\sigma}{\tau}, \rho, v\right) M(\rho, v) X(\tau, \rho, v), \quad \tau \in \Gamma,
$$

where

$$
M(\rho, v)=\lim _{\tau \rightarrow \infty} M_{(\rho, v)}^{-}(\tau)=M_{(\rho, v)}^{-}(\infty) .
$$

Our last assumption is as follows.

Assumption 4. $M(\rho, v)$ is of class $C^{2}$, and for each $\tau \in D^{+} \cup O$ the matrix function $X$ is of class $C^{2}$ as a function of $(\rho, v)$, and $\partial X / \partial \rho, \partial X / \partial v, \partial X^{\natural} / \partial \rho$ and $\partial X^{\natural} / \partial v$ are analytic as functions of $\tau$ in the domain $D^{+} \cup O$.

Note that, by Assumption 3 and (6.4), we have $X(0, \rho, v)=\mathbb{I}$ for all $(\rho, v)$ in a neighbourhood of $\left(\rho_{0}, v_{0}\right)$. Therefore

$$
\left(\frac{\partial X(\tau, \rho, v)}{\partial \rho}\right)_{\left.\right|_{\left(0, \rho_{0}, v_{0}\right)}}=\left(\frac{\partial X(0, \rho, v)}{\partial \rho}\right)_{\left.\right|_{\left(\rho_{0}, v_{0}\right)}}=0,
$$

and analogously

$$
\left(\frac{\partial X(0, \rho, v)}{\partial v}\right)_{\left.\right|_{\left(\rho_{0}, v_{0}\right)}}=0
$$


Now, for any $\left(\rho_{0}, v_{0}\right) \in S$, let

$$
C_{\rho_{0}, v_{0}}=\left\{v_{0}+\sigma \frac{\rho_{0}}{2} \frac{\sigma-\tau^{2}}{\tau}, \tau \in O \backslash F P_{\sigma}\right\} \subset \mathbb{C} \backslash W_{\rho_{0}, v_{0}},
$$

where we recall the definition of $W_{\rho_{0}, v_{0}}$ given above (3.2). For any $\omega \in C_{\rho_{0}, v_{0}}$, let $\varphi_{\omega}$ and $\widetilde{\varphi}_{\omega}$ be the two functions defined in (3.5), and let $\mathcal{T}_{\omega}=\left\{\varphi_{\omega}, \widetilde{\varphi}_{\omega}\right\}$. Thus, by definition, for any $\omega \in C_{\rho_{0}, v_{0}}, \varphi_{\omega}\left(\rho_{0}, v_{0}\right)$ and $\widetilde{\varphi}_{\omega}\left(\rho_{0}, v_{0}\right)=-\sigma / \varphi_{\omega}\left(\rho_{0}, v_{0}\right)$ lie in $O \backslash F P_{\sigma}$ and therefore, by continuity, also $\varphi_{\omega}(\rho, v)$ and $\widetilde{\varphi}_{\omega}(\rho, v)$ lie in $O \backslash F P_{\sigma}$ for all $(\rho, v)$ in a neighbourhood of $\left(\rho_{0}, v_{0}\right)$. Therefore, for all $(\rho, v)$ in a neighbourhood of $\left(\rho_{0}, v_{0}\right)$, the matrix functions $\left.X(\tau, \rho, v)\right|_{\tau=\varphi(\rho, v)}$ and $\left.X^{\natural}(-\sigma / \tau, \rho, v)\right|_{\tau=\varphi(\rho, v)}$, with $\varphi \in \mathcal{T}_{\omega}$, are well defined and are of class $C^{2}$, and the equality resulting from (6.4) by composition holds in a neighbourhood of $\left(\rho_{0}, v_{0}\right)$.

On the other hand, when replacing $\tau$ by $\varphi(\rho, v)$ of the form (3.5) in the expression for $u$ given in (6.1), one obtains $\omega$ and therefore, the matrix on the left-hand side of (6.3) becomes $\mathcal{M}(\omega)$, independent of $(\rho, v)$.

We now state the main result of this section as a theorem.

Theorem 6.1. Let Assumptions 1-4 be satisfied. Then, $M(\rho, v)$ is a solution of the field equations (2.1) on $S$.

Proof. Take any point in $S$, which we will denote by $\left(\rho_{0}, v_{0}\right)$. In the following, $(\rho, v)$ will denote an arbitrary point in a neighbourhood of $\left(\rho_{0}, v_{0}\right)$. We take the contour $\Gamma$ to have the properties described under Assumption 1. In the factorization (6.4), $\tau$ varies along $\Gamma \subset O$, while $(\rho, v)$ is kept fixed. Substituting $\tau$ by $\varphi$ of the form (3.5) with $\omega \in C_{\rho_{0}, v_{0}}$, we obtain $\mathcal{M}(\omega)$, which is independent of $(\rho, v)$, and hence differentiating with respect to $(\rho, v)$ gives zero, i.e. $d(\mathcal{M}(\omega))=0$, where $d$ denotes the differential with respect to $(\rho, v)$. Then, multiplying by $\left(X^{\natural}\right)^{-1}$ on the left and by $X^{-1}$ on the right, we obtain,

$$
\begin{aligned}
0= & {\left[\left.X^{\natural}\left(-\frac{\sigma}{\tau}, \rho, v\right)\right|_{\tau=\varphi}\right]^{-1} d\left[\left.X^{\natural}\left(-\frac{\sigma}{\tau}, \rho, v\right)\right|_{\tau=\varphi}\right] M(\rho, v) } \\
& +d M(\rho, v)+\left.M(\rho, v) d\left[\left.X(\tau, \rho, v)\right|_{\tau=\varphi}\right] X^{-1}(\tau, \rho, v)\right|_{\tau=\varphi} .
\end{aligned}
$$

Next, we multiply this system of differential equations by $\varphi+\sigma / \varphi$, where we recall that $\varphi \neq 0$ and $\varphi^{2}+\sigma \neq 0$,

$$
\begin{aligned}
0= & {\left.\left[\frac{\tau^{2}+\sigma}{\tau}\left(X^{\natural}\right)^{-1}\left(-\frac{\sigma}{\tau}, \rho, v\right)\right]\right|_{\tau=\varphi} d\left[\left.X^{\natural}\left(-\frac{\sigma}{\tau}, \rho, v\right)\right|_{\tau=\varphi}\right] M(\rho, v) } \\
& +\left.\frac{\tau^{2}+\sigma}{\tau}\right|_{\tau=\varphi} d M(\rho, v)+\left.\left.\frac{\tau^{2}+\sigma}{\tau}\right|_{\tau=\varphi} M(\rho, v) d\left(\left.X(\tau, \rho, v)\right|_{\tau=\varphi}\right) X^{-1}(\tau, \rho, v)\right|_{\tau=\varphi}
\end{aligned}
$$

We evaluate

$$
\begin{aligned}
d\left(\left.X^{\natural}\left(-\frac{\sigma}{\tau}, \rho, v\right)\right|_{\tau=\varphi}\right)= & \left.\frac{\partial X^{\natural}\left(\tau^{\prime}, \rho, v\right)}{\partial \tau^{\prime}}\right|_{\tau^{\prime}=-\frac{\sigma}{\varphi}} \frac{\sigma}{\varphi^{2}}\left(\frac{\partial \varphi}{\partial \rho} d \rho+\frac{\partial \varphi}{\partial v} d v\right) \\
& +\left.\frac{\partial X^{\natural}(\tau, \rho, v)}{\partial \rho}\right|_{\tau=-\frac{\sigma}{\varphi}} d \rho+\left.\frac{\partial X^{\natural}(\tau, \rho, v)}{\partial v}\right|_{\tau=-\frac{\sigma}{\varphi}} d v .
\end{aligned}
$$


Using

$$
d \varphi=\frac{\varphi}{\rho}\left(\frac{\sigma-\varphi^{2}}{\sigma+\varphi^{2}} d \rho+\sigma \frac{2 \varphi}{\sigma+\varphi^{2}} d v\right)
$$

(6.11) becomes

$$
\begin{aligned}
d\left(\left.X^{\natural}\left(-\frac{\sigma}{\tau}, \rho, v\right)\right|_{\tau=\varphi}\right)= & {\left[\left.\frac{\partial X^{\natural}\left(\tau^{\prime}, \rho, v\right)}{\partial \tau^{\prime}}\right|_{\tau^{\prime}=-\frac{\sigma}{\tau}} \frac{\sigma}{\tau^{2} \rho}\left(\tau \frac{\sigma-\tau^{2}}{\sigma+\tau^{2}} d \rho+2 \sigma \frac{\tau^{2}}{\sigma+\tau^{2}} d v\right)\right.} \\
& \left.+\frac{\partial X^{\natural}\left(-\frac{\sigma}{\tau}, \rho, v\right)}{\partial \rho} d \rho+\frac{\partial X^{\natural}\left(-\frac{\sigma}{\tau}, \rho, v\right)}{\partial v} d v\right]\left.\right|_{\tau=\varphi} .
\end{aligned}
$$

Next, we evaluate

$$
\begin{aligned}
d\left(\left.X(\tau, \rho, v)\right|_{\tau=\varphi}\right)= & {\left[\frac{\partial X(\tau, \rho, v)}{\partial \tau} \frac{1}{\rho}\left(\tau \frac{\sigma-\tau^{2}}{\sigma+\tau^{2}} d \rho+2 \sigma \frac{\tau^{2}}{\sigma+\tau^{2}} d v\right)\right.} \\
& \left.+\frac{\partial X(\tau, \rho, v)}{\partial \rho} d \rho+\frac{\partial X(\tau, \rho, v)}{\partial v} d v\right]\left.\right|_{\tau=\varphi}
\end{aligned}
$$

Inserting these expressions into (6.10) results in

$$
\begin{aligned}
0= & {\left[\left.\left(X^{\natural}\right)^{-1}\left(-\frac{\sigma}{\tau}, \rho, v\right) \frac{\partial X^{\natural}\left(\tau^{\prime}, \rho, v\right)}{\partial \tau^{\prime}}\right|_{\tau^{\prime}=-\frac{\sigma}{\tau}} \frac{\sigma}{\tau^{2} \rho}\left[\left(\sigma-\tau^{2}\right) d \rho+2 \sigma \tau d v\right] M(\rho, v)\right.} \\
& +\frac{\tau^{2}+\sigma}{\tau}\left(X^{\natural}\right)^{-1}\left(-\frac{\sigma}{\tau}, \rho, v\right)\left\{\frac{\partial X^{\natural}}{\partial \rho}\left(-\frac{\sigma}{\tau}, \rho, v\right) d \rho+\frac{\partial X^{\natural}}{\partial v}\left(-\frac{\sigma}{\tau}, \rho, v\right) d v\right\} M(\rho, v) \\
& +\frac{\tau^{2}+\sigma}{\tau} d M(\rho, v)+M(\rho, v)\left(\frac{\partial X}{d \tau}(\tau, \rho, v) \frac{1}{\rho}\left[\left(\sigma-\tau^{2}\right) d \rho+2 \sigma \tau d v\right]\right. \\
& \left.\left.+\frac{\tau^{2}+\sigma}{\tau}\left(\frac{\partial X}{\partial \rho}(\tau, \rho, v) d \rho+\frac{\partial X}{\partial v}(\tau, \rho, v) d v\right)\right) X^{-1}(\tau, \rho, v)\right]\left.\right|_{\tau=\varphi}
\end{aligned}
$$

Now evaluate $(6.15)$ at $\left(\rho_{0}, v_{0}\right)$. This holds for any function $\varphi$ satisfying the assumptions. Recall that any such function is labelled by $\omega \in C_{\rho_{0}, v_{0}}$. Keeping $\left(\rho_{0}, v_{0}\right)$ fixed, and varying over all $\omega \in C_{\rho_{0}, v_{0}}$ results in scanning over all values $\tau \in O \backslash F P_{\sigma}$. In this way, we can reinterpret (6.15) as an equality that holds for all $\tau \in \Gamma \backslash F P_{\sigma}$ at fixed $\left(\rho_{0}, v_{0}\right)$. For ease of notation, we now denote $\left(\rho_{0}, v_{0}\right)$ simply by $(\rho, v)$, taking into account that $\left(\rho_{0}, v_{0}\right)$ was arbitrarily fixed. Hence

$$
\begin{aligned}
0= & \left.\left(X^{\natural}\right)^{-1}\left(-\frac{\sigma}{\tau}, \rho, v\right) \frac{\partial X^{\natural}\left(\tau^{\prime}, \rho, v\right)}{\partial \tau^{\prime}}\right|_{\tau^{\prime}=-\frac{\sigma}{\tau}} \frac{\sigma}{\tau^{2} \rho}\left[\left(\sigma-\tau^{2}\right) d \rho+2 \sigma \tau d v\right] M(\rho, v) \\
& +\frac{\tau^{2}+\sigma}{\tau}\left(X^{\natural}\right)^{-1}\left(-\frac{\sigma}{\tau}, \rho, v\right)\left\{\frac{\partial X^{\natural}}{\partial \rho}\left(-\frac{\sigma}{\tau}, \rho, v\right) d \rho+\frac{\partial X^{\natural}}{\partial v}\left(-\frac{\sigma}{\tau}, \rho, v\right) d v\right\} M(\rho, v) \\
& +\frac{\tau^{2}+\sigma}{\tau} d M(\rho, v)+M(\rho, v)\left(\frac{\partial X}{d \tau}(\tau, \rho, v) \frac{1}{\rho}\left[\left(\sigma-\tau^{2}\right) d \rho+2 \sigma \tau d v\right]\right. \\
& \left.+\frac{\tau^{2}+\sigma}{\tau}\left(\frac{\partial X}{\partial \rho}(\tau, \rho, v) d \rho+\frac{\partial X}{\partial v}(\tau, \rho, v) d v\right)\right) X^{-1}(\tau, \rho, v)
\end{aligned}
$$


By continuity, this equality can also be extended to the two fixed points of $\Gamma$. Hence, (6.16) holds $\forall \tau \in \Gamma$.

We now rewrite $(6.16)$ as

$$
\begin{aligned}
- & \left.\left(X^{\natural}\right)^{-1}\left(-\frac{\sigma}{\tau}, \rho, v\right) \frac{\partial X^{\natural}\left(\tau^{\prime}, \rho, v\right)}{\partial \tau^{\prime}}\right|_{\tau^{\prime}=-\frac{\sigma}{\tau}} \frac{\sigma}{\tau^{2} \rho}\left[\left(\sigma-\tau^{2}\right) d \rho+2 \sigma \tau d v\right] M(\rho, v) \\
- & \frac{\tau^{2}+\sigma}{\tau}\left(X^{\natural}\right)^{-1}\left(-\frac{\sigma}{\tau}, \rho, v\right)\left[\frac{\partial X^{\natural}}{\partial \rho}\left(-\frac{\sigma}{\tau}, \rho, v\right) d \rho+\frac{\partial X^{\natural}}{\partial v}\left(-\frac{\sigma}{\tau}, \rho, v\right) d v\right] M(\rho, v) \\
- & \frac{\sigma}{\tau} d M(\rho, v) \\
= & \tau d M(\rho, v)+M(\rho, v) \frac{\partial X}{d \tau}(\tau, \rho, v) \frac{1}{\rho}\left[\left(\sigma-\tau^{2}\right) d \rho+2 \sigma \tau d v\right] X^{-1}(\tau, \rho, v) \\
& +\frac{\tau^{2}+\sigma}{\tau} M(\rho, v)\left(\frac{\partial X}{\partial \rho}(\tau, \rho, v) d \rho+\frac{\partial X}{\partial v}(\tau, \rho, v) d v\right) X^{-1}(\tau, \rho, v), \quad \tau \in \Gamma, \quad(6
\end{aligned}
$$

where we used Assumptions 2 and 4 to separate terms in such a manner that the left hand side of the equality is analytic in $D^{-}$(by Proposition 3.4, if $X(\tau, \cdot, \cdot)$ is analytic in $D^{+}$ then $X\left(-\frac{\sigma}{\tau}, \cdot, \cdot\right)$ is analytic in $\left.D^{-}\right)$except for a pole of order one at $\tau=\infty$, while the right hand side is analytic in $D^{+}$except for a pole of order one at $\tau=0$. Since (6.17) holds for $\tau \in \Gamma$, it constitutes a matricial Riemann-Hilbert problem that is associated to the canonical Wiener-Hopf factorization (6.4).

This associated Riemann-Hilbert problem is solved by means of a generalization of Liouville's theorem. Namely, consider a function $f$ that: equals $f_{+}$in $D^{+}$and is analytic in $D^{+}$except for a simple pole at $\tau=0$; that equals $f_{-}$in $D^{-}$and is analytic in $D^{-}$except for a simple pole at $\tau=\infty$; and that satisfies $f_{+}=f_{-}$for all $\tau$ on $\Gamma$. Then, $f$ can only be of the form

$$
f(\tau)=\frac{A \tau^{2}+B \tau+C}{\tau}
$$

where $A, B, C$ are constants.

In our case, $A, B, C$ are matrix one-forms that are independent of $\tau$. We then infer two equations from (6.17). The first one reads

$$
\begin{aligned}
& -\left.\left(X^{\natural}\right)^{-1}\left(-\frac{\sigma}{\tau}, \rho, v\right) \frac{\partial X^{\natural}\left(\tau^{\prime}, \rho, v\right)}{\partial \tau^{\prime}}\right|_{\tau^{\prime}=-\frac{\sigma}{\tau}} \frac{\sigma}{\tau^{2} \rho}\left[\left(\sigma-\tau^{2}\right) d \rho+2 \sigma \tau d v\right] M(\rho, v) \\
& -\frac{\tau^{2}+\sigma}{\tau}\left(X^{\natural}\right)^{-1}\left(-\frac{\sigma}{\tau}, \rho, v\right)\left[\frac{\partial X^{\natural}}{\partial \rho}\left(-\frac{\sigma}{\tau}, \rho, v\right) d \rho+\frac{\partial X^{\natural}}{\partial v}\left(-\frac{\sigma}{\tau}, \rho, v\right) d v\right] M(\rho, v) \\
& -\frac{\sigma}{\tau} d M(\rho, v)=\frac{A \tau^{2}+B \tau+C}{\tau}
\end{aligned}
$$

where the left hand side is analytic in $D^{-}$except for a simple pole at $\tau=\infty$. The second equation reads

$$
\begin{aligned}
\frac{A \tau^{2}+B \tau+C}{\tau}= & \tau d M(\rho, v)+M(\rho, v) \frac{\partial X}{d \tau}(\tau, \rho, v) \frac{1}{\rho}\left[\left(\sigma-\tau^{2}\right) d \rho+2 \sigma \tau d v\right] X^{-1}(\tau, \rho, v) \\
& +\frac{\tau^{2}+\sigma}{\tau} M(\rho, v)\left(\frac{\partial X}{\partial \rho}(\tau, \rho, v) d \rho+\frac{\partial X}{\partial v}(\tau, \rho, v) d v\right) X^{-1}(\tau, \rho, v),
\end{aligned}
$$


where the right hand side is analytic in $D^{+}$except for a simple pole at $\tau=0$. First we focus on (6.20) and rewrite it as

$$
\begin{aligned}
& \tau d M(\rho, v)+M(\rho, v) \frac{\partial X}{d \tau}(\tau, \rho, v) \frac{1}{\rho}\left[\left(\sigma-\tau^{2}\right) d \rho+2 \sigma \tau d v\right] X^{-1}(\tau, \rho, v) \\
& =\frac{1}{\tau}\left[-\left(\tau^{2}+\sigma\right) M(\rho, v)\left(\frac{\partial X}{\partial \rho}(\tau, \rho, v) d \rho+\frac{\partial X}{\partial v}(\tau, \rho, v) d v\right) X^{-1}(\tau, \rho, v)+A \tau^{2}+B \tau+C\right] .
\end{aligned}
$$

The left hand side of this equation is analytic in $D^{+}$, and hence the right hand side has to be analytic at $\tau=0$. Thus, the expression in the bracket on the right hand side must vanish for $\tau=0$. Now recall (6.6) and (6.7). Therefore we conclude

$$
C=0 \text {. }
$$

Applying a similar reasoning to (6.19) and demanding analyticity at $\tau=\infty$, we infer

$$
A=0
$$

Hence, we conclude that both sides (6.17) are equal to a $\tau$-independent matrix one-form $B$. Since this is valid for any $(\rho, v) \in S$, we denote $B \equiv G(\rho, v)$.

Now recall that (6.21) holds $\forall \tau \in \Gamma$, and in particular at the fixed points $\tau^{2}=-\sigma$ of the involution (3.1). Let us denote the two fixed points by $\left\{p_{F}, \tilde{p}_{F}=-p_{F}\right\}$. We introduce two projections

$$
P_{ \pm}=\frac{1}{2}\left(1 \pm \sigma p_{F} \star\right)
$$

which satisfy $P_{+}+P_{-}=\mathrm{id}, P_{+}-P_{-}=\sigma p_{F} \star, P_{ \pm}^{2}=P_{ \pm}, P_{+} P_{-}=0$. Then, using (2.3), we infer $d \rho+p_{F} d v=2 P_{-} d \rho$ as well as $\star P_{ \pm}=\mp p_{F} P_{ \pm}$.

Using these properties, we evaluate (6.21) at $\tau=p_{F}$,

$$
p_{F} d M(\rho, v)+\frac{4 \sigma}{\rho} P_{-} d \rho\left(M(\rho, v) \frac{\partial X}{d \tau}\left(p_{F}, \rho, v\right) X^{-1}\left(p_{F}, \rho, v\right)\right)=G(\rho, v) .
$$

Applying $P_{+}$to this equation, and noting that the projections only act on differential forms, results in

$$
P_{+} G(\rho, v)=p_{F} P_{+} d M(\rho, v) .
$$

On the other hand, evaluating (6.21) at $\tau=\tilde{p}_{F}=-p_{F}$ gives

$$
-p_{F} d M(\rho, v)+\frac{4 \sigma}{\rho} P_{+} d \rho\left(M(\rho, v) \frac{\partial X}{d \tau}\left(-p_{F}, \rho, v\right) X^{-1}\left(-p_{F}, \rho, v\right)\right)=G(\rho, v) .
$$

Applying $P_{-}$to this equation results in

$$
P_{-} G(\rho, v)=-p_{F} P_{-} d M(\rho, v) .
$$

Then, adding up (6.26) and (6.28) gives

$$
G(\rho, v)=-\star d M(\rho, v),
$$

while subtracting (6.26) from (6.28) does not yield new information. 
Now we return to (6.20) (with $A=C=0)$ and $B=-\star d M(\rho, v)$. Let again $\left(\rho_{0}, v_{0}\right)$ denote a point in $S$, and let $(\rho, v)$ denote any point in a neighbourhood of $\left(\rho_{0}, v_{0}\right)$, such that for $\varphi \in \mathcal{T}_{\omega}$ with $\omega \in C_{\rho_{0}, v_{0}}$ we have $\varphi(\rho, v) \subset O$. Then, substituting $\tau$ by $\varphi$ we obtain

$$
-\star d M(\rho, v)=\varphi d M(\rho, v)+\left.\left(\frac{\sigma}{\varphi}+\varphi\right) M(\rho, v) d\left[\left.X(\tau, \rho, v)\right|_{\tau=\varphi}\right] X^{-1}(\tau, \rho, v)\right|_{\tau=\varphi} .
$$

Multiplying by $M^{-1}$ on the left and by $\varphi$, this equation is equivalent to the BM linear system (4.1) by virtue of Proposition 4.1. Then, it follows by Theorem 4.2 that $M(\rho, v)$ is a solution to the field equations (2.1) on $S$.

Remark. Note that from the proof of Theorem 6.1 we moreover conclude that for $(\rho, v)$ in a neighbourhood of any $\left(\rho_{0}, v_{0}\right) \in S, M(\rho, v)$ and $\left.X(\tau, \rho, v)\right|_{\tau=\varphi}$, with $\varphi$ of the form (3.5) and with $\omega \in C_{\rho_{0}, v_{0}}$, satisfy the BM linear system (4.1).

\section{Meromorphic factorizations: a case study}

So far, we discussed canonical Wiener-Hopf factorizations of monodromy matrices. We focussed on canonical factorizations, since for these we have Theorem 6.1, which guarantees that this type of factorization yields a solution to the field equations. We do not have an analogous theorem for other types of factorizations, such as meromorphic factorizations [21]. This does not mean that those other types of factorization may not also yield solutions to the field equations. In this section, we discuss an example for which this happens. This is the example of a Kasner solution, which is a cosmological solution to Einstein's field equations in four dimensions. It belongs to the class $\sigma=-1$. We proceed as follows. We explicitly solve the BM linear system for a particular Kasner solution, and obtain the explicit expression for $X(\rho, v)$, cf. (7.9). We then use $X(\rho, v)$ to construct matrices $\mathcal{M}$ associated to this Kasner solution which are independent of the Weyl coordinates $(\rho, v)$. We then pick a particular matrix $\mathcal{M}$, which has a factorization of the form (7.14), with $M(\rho)$ corresponding to the Kasner solution. However, this factorization is not a canonical Wiener-Hopf factorization, since $X$ and $X^{\natural}$ are not analytic in the interior and exterior region, respectively, of any contour $\Gamma$ satisfying Assumption 1, although $X$ is analytic in a neighbourhood of $\tau=0$. However, the matrix $\mathcal{M}$ we picked also admits a canonical factorization. The resulting factor $M(\rho, v)$ yields a different solution of the field equations. This new solution and the Kasner solution turn out to be related by the transformation given in Proposition 3.3. Therefore, in certain circumstances, by performing a canonical factorization of $\mathcal{M}$ and applying an appropriate transformation to the associated solution, one obtains a new solution to the field equations that results from a meromorphic factorization of $\mathcal{M}$.

We consider the field equations of General Relativity in vacuum. They admit a cosmological Kasner solution given by

$$
d s_{4}^{2}=-d t^{2}+\sum_{i=1}^{3} t^{2 p_{i}} d x_{i}^{2}, t>0,
$$


where the exponents $p_{i}$ obey the following conditions:

$$
\sum_{i=1}^{3} p_{i}=1, \quad \sum_{i=1}^{3} p_{i}^{2}=1 .
$$

In the following, we take all the $p_{i}$ to be non-vanishing. Without loss of generality, we will assume that $0<p_{1}<1$.

We now bring (7.1) into Weyl-Lewis-Papapetrou form (2.4) with $\sigma=-1$,

$$
d s_{4}^{2}=\Delta d x_{3}^{2}+\Delta^{-1}\left(e^{\psi}\left(-d \rho^{2}+d v^{2}\right)+\rho^{2} d x_{2}^{2}\right),
$$

where (we take $\rho>0$ )

$$
\left\{\begin{array}{l}
\rho=t^{1-p_{1}}, \\
\Delta=\rho^{\frac{2 p_{3}}{1-p_{1}}}, \\
v=\left(1-p_{1}\right) x_{1}, \\
e^{\psi}=\frac{\rho^{\frac{2\left(p_{1}+p_{3}\right)}{1-p_{1}}}}{\left(1-p_{1}\right)^{2}} .
\end{array}\right.
$$

Note that for $t>0, \rho>0$, the function $\rho(t)=t^{1-p_{1}}$ is one-to-one.

The associated matrix $M \in G / H=\mathrm{SL}(2, \mathbb{R}) / \mathrm{SO}(1,1)$ is diagonal and takes the form

$$
M(\rho)=\left[\begin{array}{cc}
\Delta(\rho) & 0 \\
0 & \Delta^{-1}(\rho)
\end{array}\right],
$$

and the resulting matrix one-form $A=M^{-1} d M$ is given by

$$
A=\left[\begin{array}{cc}
1 & 0 \\
0 & -1
\end{array}\right] \frac{\partial_{\rho} \Delta}{\Delta} d \rho
$$

We now specialize to the case

$$
p_{1}=p_{3}=\frac{2}{3}, \quad p_{2}=-\frac{1}{3},
$$

in which case $e^{\psi}=9 \rho^{8}$ and

$$
M(\rho)=\left[\begin{array}{cc}
\rho^{4} & 0 \\
0 & \rho^{-4}
\end{array}\right] .
$$

We pick any element $\varphi \in \mathcal{T}$, cf. (3.5), and we explicitly solve the associated BM linear systen (4.1) for $X$. We obtain

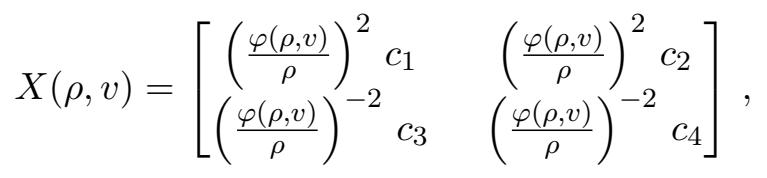

as can be verified by substituting this expression into (4.1). Here, $c_{1}, c_{2}, c_{3}, c_{4} \in \mathbb{C}$ are arbitrary integration constants. 
For the theory at hand, the involution $\downarrow$ acts as transposition on matrices. We therefore obtain for the solution $\widetilde{X}$ of the linear system (4.1) based on $\widetilde{\varphi}=1 / \varphi$,

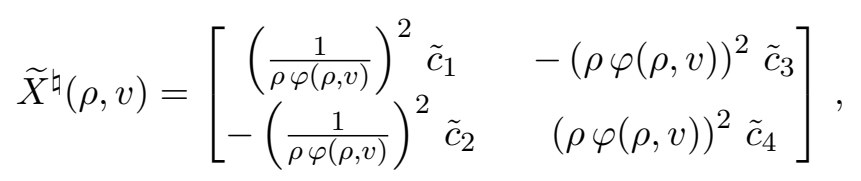

where the $\tilde{c}_{i} \in \mathbb{C}$ denote an independent set of integration constants, unrelated to the $c_{i}$. Next we compute the combination

$$
\mathcal{M}=\tilde{X}^{\natural} M X=\left[\begin{array}{ll}
\tilde{c}_{1} c_{1}-\tilde{c}_{3} c_{3} & \tilde{c}_{1} c_{2}-\tilde{c}_{3} c_{4} \\
c_{3} \tilde{c}_{4}-c_{1} \tilde{c}_{2} & \tilde{c}_{4} c_{4}-\tilde{c}_{2} c_{2}
\end{array}\right],
$$

which is independent of the Weyl coordinates $(\rho, v)$. Thus, we may obtain an arbitrary constant matrix $\mathcal{M}$, in particular the identity matrix, and the information about $M(\rho, v)$ is lost. However, as mentioned before, by choosing an appropriate matrix $\mathcal{M}$ that is independent of $(\rho, v)$, we can recover a solution $M(\rho, v)$ either by Wiener-Hopf or by meromorphic factorization. To illustrate this, we now describe how this arises in a specific example.

Let us now choose these constants as follows: $c_{2}=\tilde{c}_{2}=c_{3}=\tilde{c}_{3}=0$ and $c_{1}=\tilde{c}_{1}=$ $(2 \omega)^{2}, c_{4}=\tilde{c}_{4}=(2 \omega)^{-2}$, with $\omega \in \mathbb{C}$. Then, (7.11) becomes

$$
\mathcal{M}(\omega)=\left[\begin{array}{cc}
(2 \omega)^{4} & 0 \\
0 & (2 \omega)^{-4}
\end{array}\right]=\left[X^{\natural}\left(\frac{1}{\tau}, \rho, v\right) M(\rho) X(\tau, \rho, v)\right]_{\mid \tau=\varphi_{\omega}(\rho, v)},
$$

where, for all $(\rho, v)$ taken in a neighbourhood of $\left(\rho_{0}, v_{0}\right), \varphi_{\omega} \in \mathcal{T}$ with $\omega \neq v_{0} \pm \rho_{0}$, cf. (3.5). The expression for $X(\tau, \rho, v)$,

$$
X(\tau, \rho, v)=\left[\begin{array}{cc}
\left(2 v \frac{\tau}{\rho}+1+\tau^{2}\right)^{2} & 0 \\
0 & \left(2 v \frac{\tau}{\rho}+1+\tau^{2}\right)^{-2}
\end{array}\right]
$$

is obtained by making use of the algebraic relation (3.7) in (7.9). Also using it on the left hand side of (7.12) yields

$$
\mathcal{M}\left(v+\frac{\rho}{2} \frac{\left(1+\tau^{2}\right)}{\tau}\right)_{\mid \tau=\varphi_{\omega}(\rho, v)}=\left[X^{\natural}\left(\frac{1}{\tau}, \rho, v\right) M(\rho) X(\tau, \rho, v)\right]_{\mid \tau=\varphi_{\omega}(\rho, v)} .
$$

Now, when viewed as a factorization of $\mathcal{M}\left(v+\frac{\rho}{2} \frac{\left(1+\tau^{2}\right)}{\tau}\right)$ with respect to a contour $\Gamma,(7.14)$ describes a meromorphic factorization: although $X$ is normalized at $\tau=0$, i.e. $X(\tau=$ $0, \rho, v)=\mathbb{1}$, it has a double pole in the interior of the curve $\Gamma$, located at one of the zeroes of $2 v \frac{\tau}{\rho}+1+\tau^{2}$. The two zeroes of this quadratic polynomial in $\tau$ are at $\tau=\left(-v \pm \sqrt{v^{2}-\rho^{2}}\right) / \rho$. Let us denote them by $\tau_{1}=\left(-v+\sqrt{v^{2}-\rho^{2}}\right) / \rho$ and $\widetilde{\tau}_{1}=\left(-v-\sqrt{v^{2}-\rho^{2}}\right) / \rho$. They satisfy $\tau_{1} \widetilde{\tau}_{1}=1$. Since the curve $\Gamma$ is $\iota_{\sigma}$-invariant, then, by Proposition 3.4 , if $\tau_{1}$ is located in the interior of the curve $\Gamma, \widetilde{\tau}_{1}$ is located in the exterior region, and vice-versa (recall that $\tau_{1}$ and $\widetilde{\tau}_{1}$ cannot lie on $\Gamma$ ). Hence, we have obtained the Kasner solution (7.5) from a meromorphic factorization of $\mathcal{M}\left(v+\frac{\rho}{2} \frac{\left(1+\tau^{2}\right)}{\tau}\right)$. 
Now let us discuss the canonical factorization of the same matrix $\mathcal{M}\left(v+\frac{\rho}{2} \frac{\left(1+\tau^{2}\right)}{\tau}\right)$ with respect to $\Gamma$. If $\widetilde{\tau}_{1}$ is located in the exterior region of $\Gamma$, the resulting factors in (6.4) are

$$
M_{c}(\rho, v)=\left[\begin{array}{cc}
\left(\rho \widetilde{\tau}_{1}(\rho, v)\right)^{4} & 0 \\
0 & \left(\rho \widetilde{\tau}_{1}(\rho, v)\right)^{-4}
\end{array}\right], X_{c}(\tau, \rho, v)=\left[\begin{array}{cc}
\frac{\left(\tau-\widetilde{\tau}_{1}\right)^{4}}{\widetilde{\tau}_{1}^{4}} & 0 \\
0 & \frac{\widetilde{\tau}_{1}^{4}}{\left(\tau-\widetilde{\tau}_{1}\right)^{4}}
\end{array}\right],
$$

and analogously if $\tau_{1}$ is in the exterior region of $\Gamma$. The subscript $c$ refers to the canonical factorization. The matrix $M_{c}(\rho, v)$ in (7.15) describes a solution to the field equations (2.1) that is different from the Kasner solution $M(\rho)$ described by (7.5). Both matrices are related by

$$
M(\rho)=M_{c}(\rho, v)\left[\begin{array}{cc}
\widetilde{\tau}_{1}^{-4}(\rho, v) & 0 \\
0 & \widetilde{\tau}_{1}^{4}(\rho, v)
\end{array}\right] .
$$

This is a particular instance of the following result: we can construct new solutions to the field equations by multiplying $M_{c}(\rho, v)$ by

$$
\left[\begin{array}{cc}
K \rho^{\alpha} \varphi^{\beta} & 0 \\
0 & K^{-1} \rho^{-\alpha} \varphi^{-\beta}
\end{array}\right], \quad \alpha \in \mathbb{R}, \quad \beta \in \mathbb{Z}, \quad K \in \mathbb{R} \backslash\{0\},
$$

with $\varphi \in \mathcal{T}$. The resulting matrix $M$ as well as (7.17) satisfy the field equations (2.1) by virtue of Proposition 3.3. The case (7.16) corresponds to taking $\alpha=0, \beta=4, K=1$ and $\varphi(\rho, v)=\widetilde{\tau}_{1}(\rho, v)$. Note that neither the canonical factorization nor the solution given by (7.17) are valid on the lines $v= \pm \rho$, for which the points $\tau_{1}, \widetilde{\tau}_{1}$ coincide with the fixed points \pm 1 . However, upon multiplication by a matrix as in (7.16), we obtain a solution that is valid for all $(\rho, v)$ on the Weyl upper half-plane.

More generally, given matrix $M_{1}$ and $M_{2}$ that solve the field equations (2.1), the product $M_{1} M_{2}$ will also solve the field equations (2.1) provided that

$$
d\left(\rho M_{2}^{-1} M_{1}^{-1}\left(\star d M_{1}\right) M_{2}\right)=0,
$$

This is the case when both $M_{1}$ and $M_{2}$ are diagonal matrices, or for instance, when $M_{2}^{-1} M_{1}^{-1}\left(d M_{1}\right) M_{2}=M_{1}^{-1} d M_{1}$.

\section{The Schwarzschild monodromy matrix}

In this section, we discuss the family of solutions to the field equations of General Relativity in vacuum that results from the canonical factorization of the monodromy matrix (2.19) with $\epsilon=1$, which we call the Schwarzschild monodromy matrix,

$$
\mathcal{M}(u)=\left[\begin{array}{cc}
\sigma \frac{u-m}{u+m} & 0 \\
0 & \sigma \frac{u+m}{u-m}
\end{array}\right], \quad m \in \mathbb{R}^{+}
$$

for both cases $\sigma= \pm 1$. The involution $\downarrow$ acts as transposition on matrices. As we shall see, the case $\sigma=-1$ is more intricate than the case $\sigma=1$. 
Substituting $u$ by the expression on the right hand side of the spectral curve relation (6.1), the resulting matrix is

$$
\mathcal{M}_{(\rho, v)}(\tau)=\left[\begin{array}{cc}
\sigma \frac{\left(\tau-\tau_{1}\right)\left(\tau+\sigma / \tau_{1}\right)}{\left(\tau-\tau_{2}\right)\left(\tau+\sigma / \tau_{2}\right)} & 0 \\
0 & \sigma \frac{\left(\tau-\tau_{2}\right)\left(\tau+\sigma / \tau_{2}\right)}{\left(\tau-\tau_{1}\right)\left(\tau+\sigma / \tau_{1}\right)}
\end{array}\right]
$$

where

$\tau_{1}(\rho, v)=-\sigma \frac{m-v+\sqrt{(m-v)^{2}+\sigma \rho^{2}}}{\rho}, \quad \tau_{2}(\rho, v)=-\sigma \frac{-(m+v)+\sqrt{(m+v)^{2}+\sigma \rho^{2}}}{\rho}$,

i.e. $\tau_{1}=\varphi_{m}(\rho, v)$ and $\tau_{2}=\varphi_{-m}(\rho, v)$ if we take

$$
\varphi_{\alpha}(\rho, v)=-\sigma \frac{(\alpha-v)+\sqrt{(\alpha-v)^{2}+\sigma \rho^{2}}}{\rho}, \quad \alpha \in \mathbb{C} .
$$

Now we perform the canonical factorization of $\mathcal{M}_{(\rho, v)}(\tau)$ with respect to a closed contour $\Gamma$ in the $\tau$-plane, satisfying the following requirements:

1. $\Gamma$ passes through the fixed points of the transformation $\tau \mapsto-\frac{\sigma}{\tau}$;

2. $\Gamma$ encircles $\tau=0$;

3. if $\tau \in \Gamma$ then $-\frac{\sigma}{\tau} \in \Gamma$;

4. $\mathcal{M}_{(\rho, v)}(\tau)$ is analytic in an open set containing $\Gamma$.

For any $(\rho, v)$ in the Weyl upper half-plane such that $\tau_{1}(\rho, v), \tau_{2}(\rho, v) \notin F P_{\sigma}$ (where we recall that $F P_{\sigma}$ denotes the set of fixed points of the involution (3.1)), the contour $\Gamma$ can be chosen in such a way as to bypass $\tau_{1}$ and $\tau_{2}$. There are four possible classes of contours from which $\Gamma$ can be chosen:

(i) $\tau_{1}$ and $\tau_{2}$ are inside the contour $\Gamma$;

(ii) $\tau_{1}$ is outside and $\tau_{2}$ is inside of $\Gamma$;

(iii) both $\tau_{1}$ and $\tau_{2}$ are outside of $\Gamma$;

(iv) $\tau_{1}$ is inside and $\tau_{2}$ is outside of $\Gamma$.

Factorizing with respect to $\Gamma$ we obtain, for each of these cases,

$$
\frac{\left(\tau-\tau_{1}\right)\left(\tau+\sigma / \tau_{1}\right)}{\left(\tau-\tau_{2}\right)\left(\tau+\sigma / \tau_{2}\right)}=m_{-}(\tau) m_{+}(\tau)
$$

and

$$
M(\rho, v)=\left[\begin{array}{cc}
\sigma m_{-}(\infty) & 0 \\
0 & \sigma m_{-}^{-1}(\infty)
\end{array}\right]=\left[\begin{array}{cc}
\Delta & 0 \\
0 & \Delta^{-1}
\end{array}\right]
$$

where 
i) for a contour in class $(i)$

$$
m_{+}(\tau)=\frac{\tau_{1}}{\tau_{2}} \frac{\tau+\sigma / \tau_{1}}{\tau+\sigma / \tau_{2}}, \quad m_{-}(\tau)=\frac{\tau_{2}}{\tau_{1}} \frac{\tau-\tau_{1}}{\tau-\tau_{2}}, \quad \Delta=\sigma \tau_{2} / \tau_{1},
$$

ii) for a contour in class $(i i)$

$$
m_{+}(\tau)=\frac{1}{\tau_{1} \tau_{2}} \frac{\tau-\tau_{1}}{\tau+\sigma / \tau_{2}}, \quad m_{-}(\tau)=\tau_{1} \tau_{2} \frac{\tau+\sigma / \tau_{1}}{\tau-\tau_{2}}, \quad \Delta=\sigma \tau_{1} \tau_{2}
$$

iii) for a contour in class $($ iii $)$

$$
m_{+}(\tau)=\frac{\tau_{2}}{\tau_{1}} \frac{\tau-\tau_{1}}{\tau-\tau_{2}}, \quad m_{-}(\tau)=\frac{\tau_{1}}{\tau_{2}} \frac{\tau+\sigma / \tau_{1}}{\tau+\sigma / \tau_{2}}, \quad \Delta=\sigma \tau_{1} / \tau_{2}
$$

$i v)$ for a contour in class $(i v)$

$$
m_{+}(\tau)=\tau_{1} \tau_{2} \frac{\tau+\sigma / \tau_{1}}{\tau-\tau_{2}}, \quad m_{-}(\tau)=\frac{1}{\tau_{1} \tau_{2}} \frac{\tau-\tau_{1}}{\tau+\sigma / \tau_{2}}, \quad \Delta=\sigma \frac{1}{\tau_{1} \tau_{2}}
$$

The resulting four-dimensional space-time metrics are, in Weyl-Lewis-Papapetrou form, given by

$$
d s_{4}^{2}=-\sigma \Delta d t^{2}+\Delta^{-1}\left(e^{\psi} d s_{2}^{2}+\rho^{2} d \phi^{2}\right),
$$

with $\Delta>0$, with $d s_{2}^{2}$ given by either (2.5) or (2.6), and with $\psi$ obtained by solving (2.7).

We will now first discuss the case $\sigma=1$, and subsequently the more intricate case $\sigma=-1$.

\section{$8.1 \sigma=1$}

When $\sigma=1$, we have

$$
\tau_{1}(\rho, v)=\frac{v-m-\sqrt{(v-m)^{2}+\rho^{2}}}{\rho}, \quad \tau_{2}(\rho, v)=\frac{v+m-\sqrt{(v+m)^{2}+\rho^{2}}}{\rho},
$$

which are real for any $(\rho, v)$ in the Weyl upper half-plane. Hence, $\tau_{1}$ and $\tau_{2}$ can never coincide with the two fixed points $\pm i$ of the involution $\tau \mapsto-1 / \tau$. As a consequence, any of the solutions to the field equations obtained by canonical factorization of $\mathcal{M}_{(\rho, v)}(\tau)$ is valid in all of the Weyl upper half-plane.

The relative positions of $\tau_{1}$ and $\tau_{2}$ on the real axis of the $\tau$-plane depends on $(\rho, v)$ in the Weyl upper half-plane, as follows:

a) for $v \leq-m \Longrightarrow \tau_{1}<\tau_{2} \leq-1$

b) for $-m<v<m \Longrightarrow \tau_{1}<-1<\tau_{2}<0$;

c) for $v \geq m \Longrightarrow-1 \leq \tau_{1}<\tau_{2}<0$. 


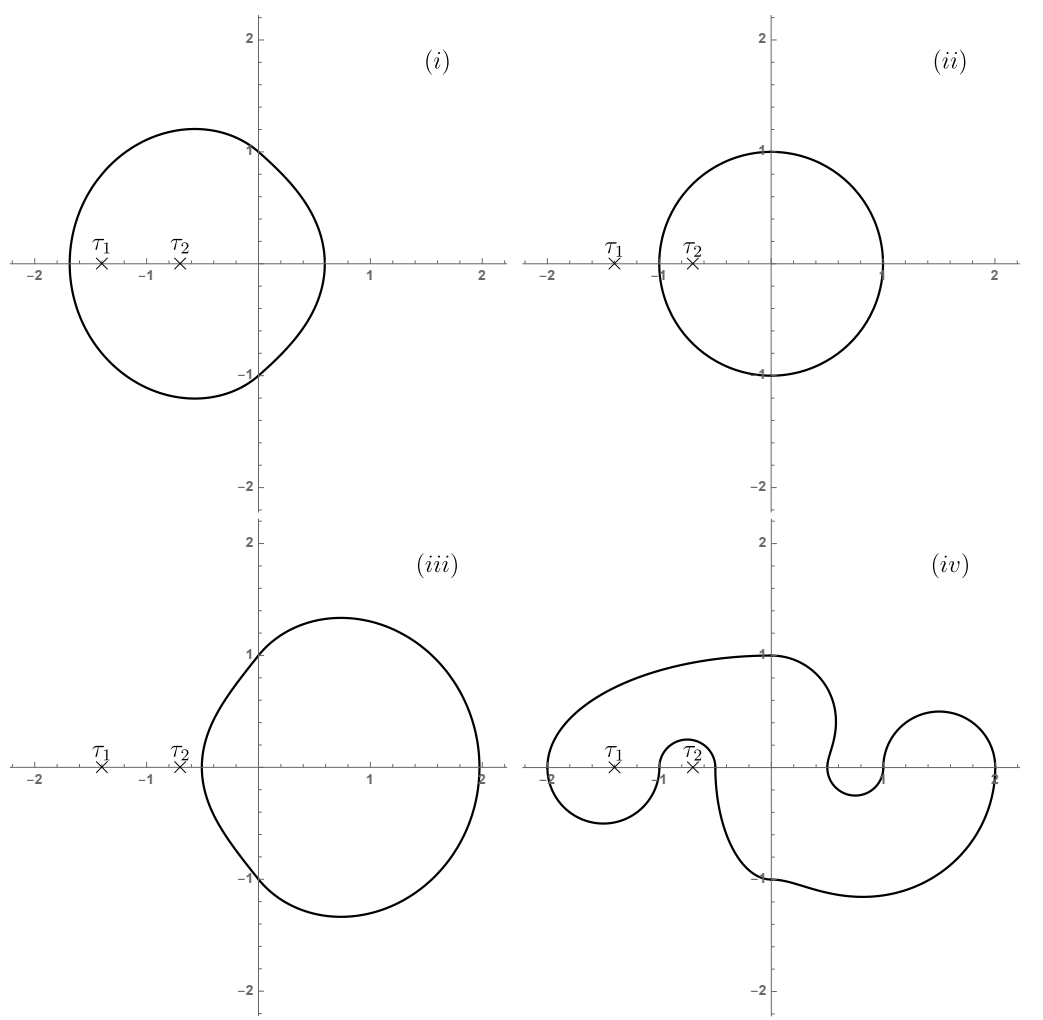

Figure 2. $-m<v<m$ : four distinct choices of contours.

Possible choices of contours for the four classes of contours $(i)-(i v)$ are depicted in figure 2 .

Next, we describe the four solutions

$$
d s_{4}^{2}=-\Delta d t^{2}+\Delta^{-1}\left(e^{\psi}\left(d \rho^{2}+d v^{2}\right)+\rho^{2} d \phi^{2}\right)
$$

that result from factorizing the Schwarzschild monodromy matrix (8.1) with $\sigma=1$ with respect to a contour $\Gamma$ belonging to one of the classes $(i)-(i v)$. To distinguish between these four solutions, we will add the subscript $i, i i, i i i, i v$ to $\Delta$ and $\psi$.

Case $(i)$ : for $\Gamma$ belonging to class $(i)$, we obtain

$$
\begin{aligned}
& \Delta_{i}(\rho, v)=\frac{v+m-\sqrt{(v+m)^{2}+\rho^{2}}}{v-m-\sqrt{(v-m)^{2}+\rho^{2}}}=\frac{v-m+\sqrt{(v-m)^{2}+\rho^{2}}}{v+m+\sqrt{(v+m)^{2}+\rho^{2}}}, \\
& \psi_{i}(\rho, v)=\log \left[\frac{1}{2} \frac{v^{2}+\rho^{2}-m^{2}}{\sqrt{(v-m)^{2}+\rho^{2}} \sqrt{(v+m)^{2}+\rho^{2}}}+\frac{1}{2}\right], \\
& K_{i}(\rho, v)=48 m^{2}\left(\frac{2}{2 m+\sqrt{(v-m)^{2}+\rho^{2}}+\sqrt{(v+m)^{2}+\rho^{2}}}\right)^{6},
\end{aligned}
$$


where $\psi=\psi_{i}$ is obtained by solving (2.7), up to a constant which we set to zero, and where $K=K_{i}$ is the Kretschmann scalar $K=R_{\mu \nu \delta \eta} R^{\mu \nu \delta \eta}$. Note that the expressions (8.14) are invariant under $v \mapsto-v$.

We note that $\Delta_{i}$ is bounded for all $(\rho, v)$ in the Weyl upper half-plane, and bounded away from zero except in a neighbourhood of the segment $\rho=0,-m<v<m$, where $\Delta_{i} \rightarrow 0$ as $\rho \rightarrow 0$ with $v$ fixed.

Using the bijection from the domain $r>2 m, 0<\theta<\pi$ in spherical coordinates onto the Weyl upper half-plane, given by

$$
\rho=\sqrt{r^{2}-2 m r} \sin \theta, \quad v=(r-m) \cos \theta,
$$

$\Delta_{i}$ becomes $\Delta_{i}=1-2 m / r$, which results in the line element

$$
d s_{4}^{2}=-\left(1-\frac{2 m}{r}\right) d t^{2}+\left(1-\frac{2 m}{r}\right)^{-1} d r^{2}+r^{2}\left(d \theta^{2}+\sin ^{2} \theta d \phi^{2}\right) .
$$

Thus, the solution (8.13) with $(\Delta, \psi)=\left(\Delta_{i}, \psi_{i}\right)$ describes the exterior region of the Schwarzschild black hole in Weyl coordinates, and the segment ] $-m, m$ [ on the $v$-axis of the Weyl upper half-plane corresponds to the Killing horizon of the Schwarzschild black hole. This solution is an example of an $A I$-metric, cf. appendix C.

Case (ii): for $\Gamma$ belonging to class $(i i)$, we obtain

$$
\begin{aligned}
& \Delta_{i i}(\rho, v)=\frac{\left(v-m-\sqrt{(v-m)^{2}+\rho^{2}}\right)\left(v+m-\sqrt{(v+m)^{2}+\rho^{2}}\right)}{\rho^{2}} \\
& \psi_{i i}(\rho, v)=\log \left[\frac{1}{2} \frac{m^{2}-v^{2}-\rho^{2}}{\left.\sqrt{(v-m)^{2}+\rho^{2}} \sqrt{(v+m)^{2}+\rho^{2}}+\frac{1}{2}\right],}\right. \\
& K_{i i}(\rho, v)=48 m^{2}\left(\frac{2}{2 m+\sqrt{(v+m)^{2}+\rho^{2}}-\sqrt{(v-m)^{2}+\rho^{2}}}\right)^{6},
\end{aligned}
$$

where $\psi=\psi_{i i}$ is obtained by solving (2.7), up to a constant which we set to zero, and where $K=K_{i i}$ is the Kretschmann scalar $K=R_{\mu \nu \delta \eta} R^{\mu \nu \delta \eta}$.

Let us consider these expressions in the limit $\rho \rightarrow 0$ with $v$ fixed. For fixed $v \in]-m, m[$, $\Delta_{i i}$ and $K_{i i}$ are bounded away from 0 , while $\psi_{i i} \rightarrow 0$. For $v>m$, we have $\Delta_{i i} \rightarrow 0, K_{i i} \rightarrow$ $3 /\left(4 m^{4}\right)$, while for $v<-m$ we have $\Delta_{i i} \rightarrow \infty, K_{i i} \rightarrow \infty$. Thus, for $\rho=0, v>m$ there is a Killing horizon, and for $\rho=0, v<-m$ a curvature singularity.

Using the change of coordinates

$$
\rho=\sqrt{2 m \varrho-\varrho^{2}} \sinh \vartheta, \quad v=(\varrho-m) \cosh \vartheta,
$$

with $\varrho \in] 0,2 m[, \vartheta \in] 0, \infty[$, the space-time metric in four dimensions takes the form

$$
d s_{4}^{2}=\left(1-\frac{2 m}{\varrho}\right) d t^{2}-\left(1-\frac{2 m}{\varrho}\right)^{-1} d \varrho^{2}+\varrho^{2}\left(d \vartheta^{2}+\sinh ^{2} \vartheta d \phi^{2}\right),
$$

which describes the 'interior' region of the AII-metric (C.3). Although this metric resembles the Schwarzschild metric (8.19), it has a different isometry group. 
Case (iii): for $\Gamma$ belonging to class $($ iii), we obtain

$$
\begin{aligned}
& \Delta_{i i i}(\rho, v)=\frac{1}{\Delta_{i}(\rho, v)}, \quad \psi_{i i i}(\rho, v)=\psi_{i}(\rho, v), \\
& K_{i i i}(\rho, v)=48 m^{2}\left(\frac{2}{2 m-\sqrt{(v-m)^{2}+\rho^{2}}-\sqrt{(v+m)^{2}+\rho^{2}}}\right)^{6} .
\end{aligned}
$$

Using the bijection from the domain $r>0,0<\theta<\pi$ in spherical coordinates onto the Weyl upper half-plane, given by

$$
\rho=\sqrt{r^{2}+2 m r} \sin \theta, \quad v=(r+m) \cos \theta,
$$

results in the line element

$$
d s_{4}^{2}=-\left(1+\frac{2 m}{r}\right) d t^{2}+\left(1+\frac{2 m}{r}\right)^{-1} d r^{2}+r^{2}\left(d \theta^{2}+\sin ^{2} \theta d \phi^{2}\right) .
$$

This is the 'negative mass' Schwarzschild solution [8], which has a naked curvature singularity at $r=0$. This solution is an example of an $A I$-metric, cf. appendix C.

Case $(\boldsymbol{i v})$ : we note that the transformation $\tau_{1} \tau_{2} \mapsto 1 /\left(\tau_{1} \tau_{2}\right)$ is effected by mapping $v \mapsto-v$. Hence, for $\Gamma$ belonging to class $(i v)$, we obtain

$$
\begin{aligned}
& \Delta_{i v}(\rho, v)=\frac{1}{\Delta_{i i}(\rho, v)}, \quad \psi_{i v}(\rho, v)=\psi_{i i}(\rho, v), \\
& K_{i v}(\rho, v)=48 m^{2}\left(\frac{2}{2 m+\sqrt{(v-m)^{2}+\rho^{2}}-\sqrt{(v+m)^{2}+\rho^{2}}}\right)^{6} .
\end{aligned}
$$

Since (8.17) and (8.23) are related by the coordinate transformation $v \mapsto-v$, the solution described by (8.23) is the same as (8.19).

\section{$8.2 \sigma=-1$}

When $\sigma=-1$ we have, from (8.3),

$$
\tau_{1}(\rho, v)=\frac{m-v+\sqrt{(m-v)^{2}-\rho^{2}}}{\rho}, \quad \tau_{2}(\rho, v)=\frac{-(m+v)+\sqrt{(m+v)^{2}-\rho^{2}}}{\rho} .
$$

Note that, differently from the case $\sigma=1, \tau_{1}$ and $\tau_{2}$ can now take complex values and may, moreover, coincide with the fixed points $\pm 1 \in F P_{-1}$ through which the curve $\Gamma$ has to pass. This occurs for $(\rho, v)$ on four half-lines, namely

$$
\begin{array}{lll}
\rho= \pm(v-m), & \text { where } & \tau_{1}=\mp 1, \\
\rho= \pm(v+m), & \text { where } & \tau_{2}=\mp 1 .
\end{array}
$$

Imposing that $\tau_{1}$ and $\tau_{2}$ are real, so as to ensure that the solutions obtained from the canonical factorization of $\mathcal{M}_{(\rho, v)}(\tau)$ are real, we find that we have to restrict $(\rho, v)$ to lie 


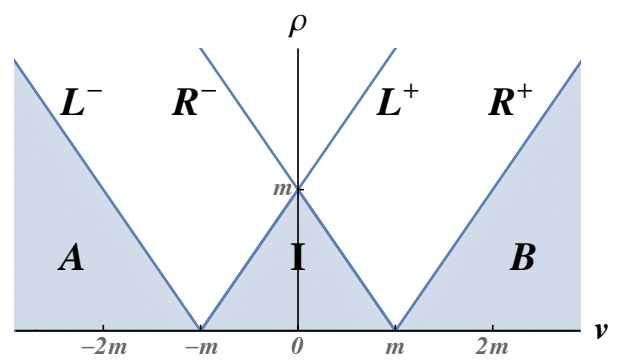

Figure 3. Regions in the Weyl upper-half plane for which both $\tau_{1}$ and $\tau_{2}$ are real.

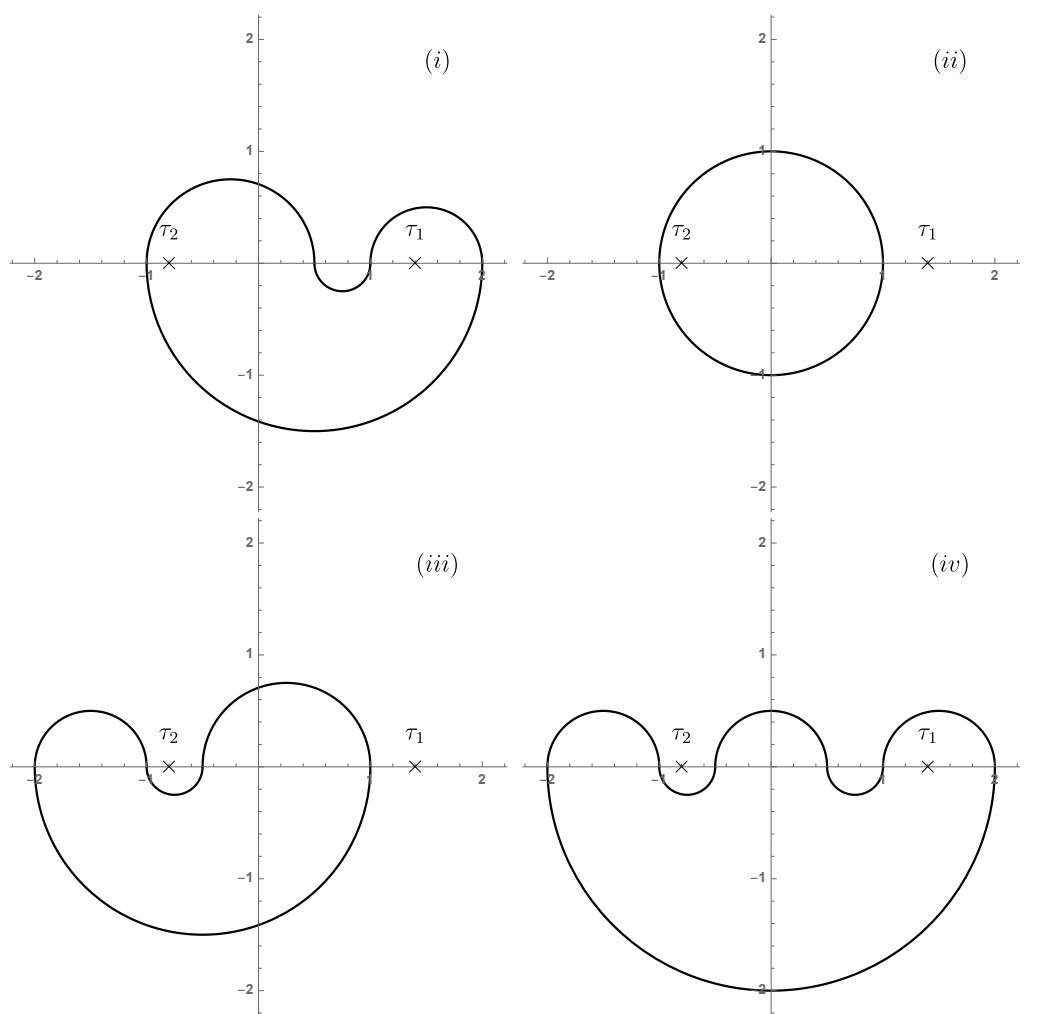

Figure 4. Four distinct choices of contours.

in the open set consisting of the three regions in the Weyl upper-half plane denoted by $I, A, B$ in figure 3. In figure 3 , we denote by $L_{ \pm}$the lines described by $\rho= \pm(v+m)$ and by $R_{ \pm}$the lines described by $\rho= \pm(v-m)$.

Considering now the cases $(i)-(i v)$ corresponding to the various possible choices of the contour $\Gamma$, as described at the beginning of section 8 , we start by noting that in each of the cases $(i)-(i v)$ we have $\Delta>0$ if $(\rho, v)$ is taken to lie in region $I$, while $\Delta<0$ for region $A$ and region $B$.

We will first consider the case when $(\rho, v)$ takes values in region $I$, in which case $\tau_{1}>1$ and $-1<\tau_{2}<0$. Possible choices of contours for the four classes of contours $(i)-(i v)$ are depicted in figure 4 . 


\subsubsection{Region $I$}

Here we take $\rho$ to be a time-like coordinate. Accordingly, we take the four-dimensional space-time metric to be given by (8.11) and (2.5) (with $\sigma=-1$ ), i.e.

$$
d s_{4}^{2}=\Delta d t^{2}+\Delta^{-1}\left(e^{\psi}\left(-d \rho^{2}+d v^{2}\right)+\rho^{2} d \phi^{2}\right) .
$$

We obtain the following expressions for $\Delta$ and $\psi$ and for the four-dimensional Kretschmann scalar $K$ :

Case $(\boldsymbol{i})$ : for $\Gamma$ belonging to class $(i)$, we obtain

$$
\begin{aligned}
& \Delta_{i}(\rho, v)=-\frac{\tau_{2}(\rho, v)}{\tau_{1}(\rho, v)}=\frac{m+v-\sqrt{(m+v)^{2}-\rho^{2}}}{m-v+\sqrt{(m-v)^{2}-\rho^{2}}}=\frac{m-v-\sqrt{(m-v)^{2}-\rho^{2}}}{m+v+\sqrt{(m+v)^{2}-\rho^{2}}} \\
& \psi_{i}(\rho, v)=\log \left[\frac{1}{2} \mid \frac{m^{2}-v^{2}+\rho^{2}}{\left.\sqrt{(m-v)^{2}-\rho^{2}} \sqrt{(m+v)^{2}-\rho^{2}}-1 \mid\right]}\right. \\
& K_{i}(\rho, v)=48 m^{2}\left(\frac{2}{2 m+\sqrt{(m+v)^{2}-\rho^{2}}+\sqrt{(m-v)^{2}-\rho^{2}}}\right)^{6},
\end{aligned}
$$

where $\psi=\psi_{i}$ is obtained by solving (2.7), up to a constant which we set to zero. Note that the expressions (8.27) are invariant under $v \mapsto-v$.

For $(\rho, v)$ taking values in region $I$, we obtain the following boundary values: on $R_{-}$,

$$
\begin{aligned}
\Delta_{i} & =\frac{2 m-\rho-\sqrt{(2 m-\rho)^{2}-\rho^{2}}}{\rho}, \quad \text { (bounded, non - zero) } \\
\psi_{i} & \rightarrow \infty, \\
K_{i} & =48 m^{2}\left(\frac{1}{m+\sqrt{(2 m-\rho)^{2}-\rho^{2}}}\right)^{6},
\end{aligned}
$$

while on $L_{+}$

$$
\begin{aligned}
\Delta_{i} & =\frac{\rho}{2 m-\rho+\sqrt{(2 m-\rho)^{2}-\rho^{2}}}, \quad \text { (bounded, non - zero) } \\
\psi_{i} & \rightarrow \infty, \\
K_{i} & =48 m^{2}\left(\frac{1}{m+\sqrt{(2 m-\rho)^{2}-\rho^{2}}}\right)^{6},
\end{aligned}
$$

and for $\rho=0$ and $-m<v<m$,

$$
\begin{aligned}
\Delta_{i} & =0, \\
\psi_{i} & \rightarrow-\infty, \\
K_{i} & =\frac{3}{4 m^{4}} .
\end{aligned}
$$

This solution has thus a Killing horizon on the segment $\rho=0,-m<v<m$. 
Case $(\mathbf{i i})$ : for $\Gamma$ belonging to class $(i i)$, we obtain

$$
\begin{aligned}
& \left.\Delta_{i i}(\rho, v)=-\tau_{1}(\rho, v) \tau_{2}(\rho, v)=\frac{\left(m-v+\sqrt{(m-v)^{2}-\rho^{2}}\right)\left(m+v-\sqrt{(m+v)^{2}-\rho^{2}}\right)}{\rho^{2}}+1 \mid\right] \\
& \psi_{i i}(\rho, v)=\log \left[\frac{1}{2} \mid \frac{m^{2}-v^{2}+\rho^{2}}{\sqrt{(m-v)^{2}-\rho^{2}} \sqrt{(m+v)^{2}-\rho^{2}}},\right. \\
& K_{i i}(\rho, v)=48 m^{2}\left(\frac{2}{2 m+\sqrt{(m+v)^{2}-\rho^{2}}-\sqrt{(m-v)^{2}-\rho^{2}}}\right)^{6}
\end{aligned}
$$

where $\psi=\psi_{i i}$ is obtained by solving (2.7), up to a constant which we set to zero.

For $(\rho, v)$ taking values in region $I$, we obtain the following boundary values: on $R_{-}$, they coincide with those for the case $(i)$ given in (8.28), while on $L_{+}$

$$
\begin{aligned}
\Delta_{i i} & =\frac{2 m-\rho+\sqrt{(2 m-\rho)^{2}-\rho^{2}}}{\rho}, \quad \text { (bounded, non - zero) } \\
\psi_{i i} & \rightarrow \infty, \\
K_{i i} & =48 m^{2}\left(\frac{1}{m-\sqrt{-m v}}\right)^{6},
\end{aligned}
$$

and for $\rho=0$ and $-m<v<m$,

$$
\begin{aligned}
\Delta_{i i} & =\frac{m-v}{m+v}, \\
\psi_{i i} & =0, \\
K_{i i} & =\frac{48 m^{2}}{(m+v)^{6}} .
\end{aligned}
$$

This solution has no Killing horizon and no curvature singularity.

Case (iii): for $\Gamma$ belonging to class (iii), we obtain

$$
\begin{aligned}
& \Delta_{i i i}(\rho, v)=\frac{1}{\Delta_{i}(\rho, v)}, \quad \psi_{i i i}(\rho, v)=\psi_{i}(\rho, v) \\
& K_{i i i}(\rho, v)=48 m^{2}\left(\frac{2}{2 m-\sqrt{(m-v)^{2}-\rho^{2}}-\sqrt{(m+v)^{2}-\rho^{2}}}\right)^{6} .
\end{aligned}
$$

For $(\rho, v)$ taking values in region $I$, we obtain the following boundary values: on $R_{-}$,

$$
\begin{aligned}
\Delta_{i i i} & =\frac{1}{\Delta_{i}}, \\
\psi_{i i i} & \rightarrow \infty \\
K_{i i i} & =48 m^{2}\left(\frac{2}{2 m-\sqrt{(2 m-\rho)^{2}-\rho^{2}}}\right)^{6},
\end{aligned}
$$


while on $L_{+}$

$$
\begin{aligned}
\Delta_{i i i} & =\frac{1}{\Delta_{i}}, \\
\psi_{i i i} & \rightarrow \infty \\
K_{i i i} & =48 m^{2}\left(\frac{2}{2 m-\sqrt{(2 m-\rho)^{2}-\rho^{2}}}\right)^{6},
\end{aligned}
$$

and for $\rho=0$ and $-m<v<m$,

$$
\begin{aligned}
\Delta_{i i i} & \rightarrow \infty, \\
\psi_{i i i} & \rightarrow-\infty, \\
K_{i i i} & \rightarrow \infty .
\end{aligned}
$$

Thus, this solution has a curvature singularity on the segment $\rho=0,-m<v<m$.

Case $(\boldsymbol{i v})$ : we note that the transformation $\tau_{1} \tau_{2} \mapsto 1 /\left(\tau_{1} \tau_{2}\right)$ is effected by mapping $v \mapsto-v$. Hence, for $\Gamma$ belonging to class $(i v)$, we obtain

$$
\begin{aligned}
& \Delta_{i v}(\rho, v)=\frac{1}{\Delta_{i i}(\rho, v)}, \quad \psi_{i v}(\rho, v)=\psi_{i i}(\rho, v), \\
& K_{i v}(\rho, v)=48 m^{2}\left(\frac{2}{2 m+\sqrt{(m-v)^{2}-\rho^{2}}-\sqrt{(m+v)^{2}-\rho^{2}}}\right)^{6} .
\end{aligned}
$$

For $(\rho, v)$ taking values in region $I$, we obtain the following boundary values: on $R_{-}$,

$$
\begin{aligned}
\Delta_{i v} & =\frac{1}{\Delta_{i i}}, \\
\psi_{i v} & \rightarrow \infty \\
K_{i v} & =48 m^{2}\left(\frac{2}{2 m-\sqrt{(2 m-\rho)^{2}-\rho^{2}}}\right)^{6},
\end{aligned}
$$

while on $L_{+}$

$$
\begin{aligned}
\Delta_{i v} & =\frac{1}{\Delta_{i i}}, \\
\psi_{i v} & \rightarrow \infty \\
K_{i v} & =48 m^{2}\left(\frac{2}{2 m+\sqrt{(2 m-\rho)^{2}-\rho^{2}}}\right)^{6},
\end{aligned}
$$

and for $\rho=0$ and $-m<v<m$,

$$
\begin{aligned}
\Delta_{i v} & =\frac{1}{\Delta_{i i}}, \\
\psi_{i v} & =0 \\
K_{i v} & =\frac{48 m^{2}}{(m-v)^{6}} .
\end{aligned}
$$

This solution has no Killing horizon and no curvature singularity. 


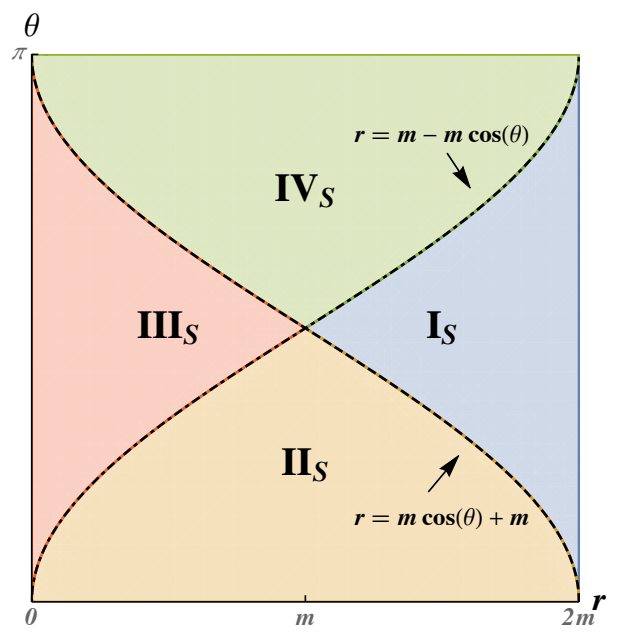

Figure 5. Bijection to quadrangle in $(r, \cos \theta)$-plane.

\subsubsection{Extending solutions: the interior region of the Schwarzschild solution}

In subsection 8.1 we used the change to spherical coordinates (8.15) to show that one of the solutions to the field equations that we obtained in Weyl coordinates describes the exterior region of the Schwarzschild black hole. If we write (8.15) as

$$
\rho=\sqrt{\left|r^{2}-2 m r\right|} \sin \theta, \quad v=(r-m) \cos \theta,
$$

it can be verified that (8.42), in the form

$$
\rho=\sqrt{2 m r-r^{2}} \sin \theta, \quad v=(r-m) \cos \theta,
$$

maps bijectively the region $I_{S}$, contained in the $(r, \theta)$-rectangle with $0<r<2 m, 0<\theta<\pi$, defined by

$$
m-r<m \cos \theta<r-m,
$$

onto the $(\rho, v)$-triangle $I$, see figure 5 . With the change of coordinates (8.43), the metric component $\Delta_{i}$ for case $(i)$ with $\sigma=-1$ becomes $\Delta_{i, S}=2 m / r-1$, and the associated four-dimensional metric (8.26) takes the form

$$
d s_{4}^{2}=\left(\frac{2 m}{r}-1\right) d t^{2}-\left(\frac{2 m}{r}-1\right)^{-1} d r^{2}+r^{2}\left(d \theta^{2}+\sin ^{2} \theta d \phi^{2}\right)
$$

i.e. it coincides on $I_{S}$ with the metric describing the interior region of the Schwarzschild solution.

It is thus natural to ask whether it is possible to extend $\Delta_{i}$ in region $I$ into neighbouring regions of the Weyl upper-half plane in a continuous manner, in such a way as to recover the whole interior through appropriate changes of coordinates. This extension can indeed be implemented by performing affine coordinate changes (3.18) of Weyl coordinates. The whole interior region of the Schwarzschild solution is obtained by extending the solution in region $I$ to the quadrangle depicted in figure 6 , keeping $\rho$ as the time-like coordinate. We now proced to explain this extension. 


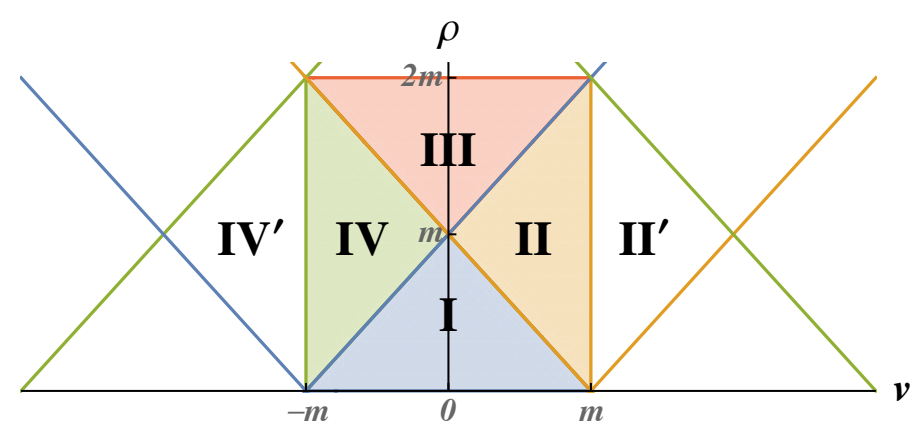

Figure 6. Quadrangle $0<\rho<2 m,-m<v<m$.

\begin{tabular}{|l|l|l|l|}
\hline$A$ & $I \rightarrow I$ & $(\rho, v) \mapsto(\rho, v)$ & $\rho=0 \mapsto \rho=0$ \\
\hline$B$ & $I \rightarrow I I$ & $(\rho, v) \mapsto(m-v, m-\rho)$ & $\rho=0 \mapsto v=m$ \\
\hline$C$ & $I \rightarrow I I I$ & $(\rho, v) \mapsto(2 m-\rho,-v)$ & $\rho=0 \mapsto \rho=2 m$ \\
\hline$D$ & $I \rightarrow I V$ & $(\rho, v) \mapsto(m+v,-m+\rho)$ & $\rho=0 \mapsto v=-m$ \\
\hline
\end{tabular}

Table 1. Bijections between triangles.

We can define bijections from the triangle $I$ onto each of the four triangles $I, I I, I I I, I V$ represented in figure 6 . This is implemented using the affine coordinate transformations given in table 1 . Note that the affine transformations are such that they leave the lines $\rho=m+v$ and $\rho=m-v$ invariant.

Taking into account the results of subsection 8.2.1, we may therefore extend $\Delta_{i}(\rho, v)$ continuously to the rectangle $-m<v<m, 0<\rho<2 m$ by

$$
\Delta(\rho, v)= \begin{cases}\Delta_{i}(\rho, v) \text { on } I \\ \Delta_{i i}\left(B^{-1}(\rho, v)\right) & \text { on } I I \\ \Delta_{i i i}\left(C^{-1}(\rho, v)\right) & \text { on } I I I \\ \Delta_{i v}\left(D^{-1}(\rho, v)\right) & \text { on } I V\end{cases}
$$

where $\Delta_{i i}\left(B^{-1}(\rho, v)\right)$ and $\Delta_{i v}\left(D^{-1}(\rho, v)\right)$ can in turn be further extended naturally to the triangles $I I^{\prime}$ and $I V^{\prime}$, respectively, in figure 6 , since

$$
\begin{gathered}
\Delta_{i i}\left(B^{-1}(\rho, v)\right)=-\frac{\left(\rho+\sqrt{\rho^{2}-(m-v)^{2}}\right)\left(\rho-2 m+\sqrt{(\rho-2 m)^{2}-(m-v)^{2}}\right)}{(m-v)^{2}}, \\
\Delta_{i v}\left(D^{-1}(\rho, v)\right)=-\frac{\left(\rho+\sqrt{\rho^{2}-(m+v)^{2}}\right)\left(\rho-2 m+\sqrt{(\rho-2 m)^{2}-(m+v)^{2}}\right)}{(m+v)^{2}},
\end{gathered}
$$

are well-behaved and positive also in these regions.

In order to ensure that $\rho$ is a time-like coordinate in the quadrangle depicted in figure 6 , the affine transformations of table 1 from region $I$ into regions $I I$ and $I V$ need to be accompanied by the transformation $d s_{2}^{2} \rightarrow-d s_{2}^{2}$, which is consistent with the fact that $d s_{2}^{2}$ can take the form (2.5) or (2.6). 


\begin{tabular}{|l|l|}
\hline$I \rightarrow I_{S}$ & $\rho=\sqrt{2 m r-r^{2}} \sin \theta, \quad v=(r-m) \cos \theta$ \\
\hline$I I \rightarrow I I_{S}$ & $\rho=m-(r-m) \cos \theta, \quad v=m-\sqrt{2 m r-r^{2}} \sin \theta$ \\
\hline$I I I \rightarrow I I I_{S}$ & $\rho=2 m-\sqrt{2 m r-r^{2}} \sin \theta, \quad v=(m-r) \cos \theta$ \\
\hline$I V \rightarrow I V_{S}$ & $\rho=m+(r-m) \cos \theta, \quad v=-m+\sqrt{2 m r-r^{2}} \sin \theta$ \\
\hline
\end{tabular}

Table 2. Bijective transformations.

Then, expressing (8.46) in spherical coordinates $(r, \theta)$, with $0<\theta<\pi, 0<r<2 m$, according to table 2, we obtain (8.45), which describes the whole interior region of the Schwarzschild black hole. This solution is an example of an $A I$-metric, cf. appendix C.

If we now consider $\Delta_{i i}$ or $\Delta_{i i i}$ instead of $\Delta_{i}$ in triangle $I$ of the Weyl upper-half plane, we obtain, by an appropriate change of coordinates, the Schwarzschild metric (8.45) in one of the triangles of figure 5 , which we can then extend in an analogous manner. As an example, consider $\Delta_{i i}$ in region $I$ and subject it to the following sequence of coordinate transformations,

$$
(\rho, v) \mapsto(m-v, m-\rho) \mapsto\left(\sqrt{2 m r-r^{2}} \sin \theta,(r-m) \cos \theta\right) .
$$

This yields the Schwarzschild solution (8.45) in region $I I_{S}$, see figure 5, provided the transformation (8.48) is accompanied by the transformation $d s_{2}^{2} \rightarrow-d s_{2}^{2}$, to ensure that $\rho$ is a time-like coordinate.

Clearly, we could have defined other continuous extensions of $\Delta_{i}$ in region $I$, keeping $\rho$ as the time-like coordinate, by using the same affine transformations. There are three such possible extensions when taking into account the behaviour of $\Delta_{i}$ on the boundary lines $\rho= \pm v+m$. They are given by

$$
\begin{aligned}
& \underbrace{\Delta_{i}(\rho, v)}_{\text {in region } I} \rightarrow \underbrace{\Delta_{i}\left(B^{-1}(\rho, v)\right)}_{\text {in region } I I} \rightarrow \underbrace{\Delta_{i}\left(C^{-1}(\rho, v)\right)}_{\text {in region } I I I} \rightarrow \underbrace{\Delta_{i}\left(D^{-1}(\rho, v)\right)}_{\text {in region } I V} \\
& \underbrace{\Delta_{i}(\rho, v)}_{\text {in region } I} \rightarrow \underbrace{\Delta_{i}\left(B^{-1}(\rho, v)\right)}_{\text {in region } I I} \rightarrow \underbrace{\Delta_{i v}\left(C^{-1}(\rho, v)\right)}_{\text {in region } I I I} \rightarrow \underbrace{\Delta_{i v}\left(D^{-1}(\rho, v)\right)}_{\text {in region } I V} \\
& \underbrace{\Delta_{i}(\rho, v)}_{\text {in region } I} \rightarrow \underbrace{\Delta_{i i}\left(B^{-1}(\rho, v)\right)}_{\text {in region } I I} \rightarrow \underbrace{\Delta_{i i}\left(C^{-1}(\rho, v)\right)}_{\text {in region } I I I} \rightarrow \underbrace{\Delta_{i}\left(D^{-1}(\rho, v)\right)}_{\text {in region } I V}
\end{aligned}
$$

However, unlike the extension (8.46), these three extensions have a jump in the transverse extrinsic curvature $[12,25]$ across the lines $\rho= \pm v+m$. We discuss this in appendix B.

\subsubsection{Regions $A$ and $B$}

Now we take $(\rho, v)$ to lie in regions $A$ and $B$ in figure 3 . Then, as mentioned at the beginning of subsection 8.2, from the factorization of $\mathcal{M}_{(\rho, v)}(\tau)$ with $\sigma=-1$ we obtain four different solutions $\Delta(\rho, v)$, corresponding to the cases $(i)-(i v)$, with $\Delta<0$. Although this violates our initial assumption that $\Delta>0$ (see (2.4)), this is easily overcome by changing the overall sign of the monodromy matrix (8.1). This implies that we can take over the expressions 


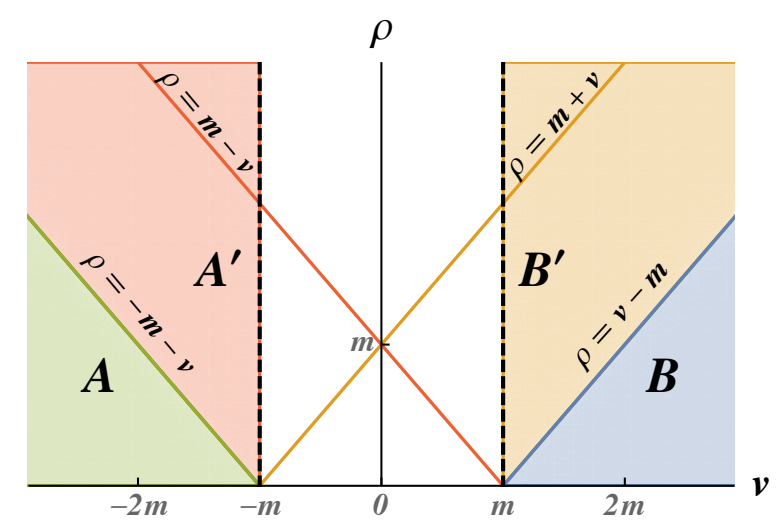

Figure 7. Weyl upper-half plane with solutions extended past regions $A$ and $B$.

for $\Delta, \psi$ and $K$ for the cases $(i)$ - (iv) of subsection 8.2, merely changing the sign of the expression for $\Delta$.

We begin by considering solutions in region $A$.

First case: let us first consider the solution $\Delta_{i v}^{A}=\frac{1}{\tau_{1} \tau_{2}}$, where $\tau_{1}$ and $\tau_{2}$ are given by $(8.24)$,

$$
\Delta_{i v}^{A}(\rho, v)=\frac{\rho^{2}}{\left(m-v+\sqrt{(m-v)^{2}-\rho^{2}}\right)\left(-(m+v)+\sqrt{(m+v)^{2}-\rho^{2}}\right)} .
$$

This solution possesses a Killing horizon for $\rho=0, v<-m$ (where $\Delta_{i v}^{A}(\rho, v)=0$ ). By applying the affine transformation $(\rho, v) \mapsto(-m-v,-m-\rho)$ to the solution $\Delta_{i}^{A}=\tau_{2} / \tau_{1}$,

$$
\Delta_{i}^{A}(\rho, v)=\frac{-(m+v)+\sqrt{(m+v)^{2}-\rho^{2}}}{m-v+\sqrt{(m-v)^{2}-\rho^{2}}},
$$

we obtain a solution in region $A^{\prime}$ (represented in figure 7) which continuously extends $\Delta_{i v}^{A}$ to $A \cup A^{\prime}$, and is actually also defined and valid in the region $A^{\prime \prime}$ which comprises the points between the lines $v=-m$ and $\rho=v+m$.

If we now consider coordinates $(\varrho, \vartheta) \in] 2 m, \infty[\times] 0, \infty[$ and divide this domain into regions

$$
\begin{aligned}
\tilde{A} & =\{(\varrho, \vartheta): \varrho>2 m, \vartheta>0, \varrho<m+m \cosh \vartheta\}, \\
\tilde{A}^{\prime} & =\{(\varrho, \vartheta): \varrho>2 m, \vartheta>0, \varrho>m+m \cosh \vartheta\},
\end{aligned}
$$

we see that $\tilde{A}$ is bijectively mapped onto $A$ by

$$
\rho=\sqrt{\varrho^{2}-2 m \varrho} \sinh \vartheta, \quad v=(m-\varrho) \cosh \vartheta,
$$

and $\tilde{A}^{\prime}$ is bijectively mapped onto $A^{\prime}$ by

$$
\rho=-m-(m-\varrho) \cosh \vartheta, \quad v=-m-\sqrt{\varrho^{2}-2 m \varrho} \sinh \vartheta .
$$


With this change of coordinates, the previous solution given by

$$
\Delta(\rho, v)=\left\{\begin{array}{l}
\Delta_{i v}^{A}(\rho, v) \quad \text { on } A, \\
\Delta_{i}^{A}(-m-v,-m-\rho) \quad \text { on } A^{\prime},
\end{array}\right.
$$

becomes

$$
\Delta(\rho, v)=1-\frac{2 m}{\varrho},
$$

As before, to ensure that $\rho$ is a time-like coordinate in region $A^{\prime}$, the affine transformation $(\rho, v) \mapsto(-m-v,-m-\rho)$ needs to be accompanied by the transformation $d s_{2}^{2} \rightarrow-d s_{2}^{2}$. The resulting four-dimensional metric reads

$$
d s_{4}^{2}=\left(1-\frac{2 m}{\varrho}\right) d t^{2}-\left(1-\frac{2 m}{\varrho}\right)^{-1} d \varrho^{2}+\varrho^{2}\left(d \vartheta^{2}+\sinh ^{2} \vartheta d \phi^{2}\right),
$$

which describes the 'exterior' region of the $A I I$-metric (C.3).

Second case: in an analogous manner, we can extend the solution $\Delta_{i i}^{A}=1 /\left(\Delta_{i v}^{A}\right)$ in region $A$ to region $A \cup A^{\prime} \cup A^{\prime \prime}$ by

$$
\Delta(\rho, v)=\left\{\begin{array}{l}
\Delta_{i i}^{A}(\rho, v) \quad \text { on } A, \\
\Delta_{i i i}^{A}(-m-v,-m-\rho) \quad \text { on } A^{\prime} \cup A^{\prime \prime} .
\end{array}\right.
$$

The associated space-time metric has a curvature singularity at $\rho=0, v<-m$. By using the change of coordinates

$$
\rho=\sqrt{\varrho^{2}+2 m \varrho} \sinh \vartheta, \quad v=-(m+\varrho) \cosh \vartheta,
$$

defined for $\varrho>0, \vartheta>0$, the regions $\{(\varrho, \vartheta): \varrho>0, \vartheta>0, \varrho<-m+m \cosh \vartheta\}$ and $\{(\varrho, \vartheta)$ : $\varrho>0, \vartheta>0, \varrho>-m+m \cosh \vartheta\}$ are bijectively mapped into $A$ and $A^{\prime}$, respectively, and in these new coordinates $\Delta(\rho, v)$ is given by $\Delta(\rho, v)=1+2 m / \varrho$, which results in the four-dimensional metric

$$
d s_{4}^{2}=\left(1+\frac{2 m}{\varrho}\right) d t^{2}-\left(1+\frac{2 m}{\varrho}\right)^{-1} d \varrho^{2}+\varrho^{2}\left(d \vartheta^{2}+\sinh ^{2} \vartheta d \phi^{2}\right) .
$$

This is the 'negative mass' version of the $A I I$-metric (8.57) [8], cf. appendix C.

Note that the other two vacuum solutions on $A \cup A^{\prime}$ given by

$$
\begin{aligned}
& \Delta(\rho, v)= \begin{cases}\Delta_{i}^{A}(\rho, v) & \text { on } A, \\
\Delta_{i v}^{A}(-m-v,-m-\rho) & \text { on } A^{\prime}\end{cases} \\
& \Delta(\rho, v)= \begin{cases}\Delta_{i i i}^{A}(\rho, v) & \text { on } A, \\
\Delta_{i i}^{A}(-m-v,-m-\rho) & \text { on } A^{\prime}\end{cases}
\end{aligned}
$$

are identical to (8.55) and (8.58), respectively, via $(\rho, v) \mapsto(-m-v,-m-\rho)$.

Now let us consider solutions defined in region $B$. We note that solutions on $B \cup B^{\prime}$ obtained in an analogous manner as above are mapped into the ones on $A \cup A^{\prime}$ discussed above, so that we do not have to discuss them separately.

Finally, we note that, as in (8.49), we can define other continuous extensions of $\Delta_{i}^{A}, \ldots, \Delta_{i v}^{A}$. These other extensions will suffer from a jump in the transverse extrinsic curvature across the line $\rho=-m-v$. 


\section{The monodromy matrix with $\epsilon=0$}

In this section, we discuss the canonical factorization of the monodromy matrix (2.19) with $\epsilon=0$,

$$
\mathcal{M}(u)=\left[\begin{array}{cc}
\sigma \frac{u}{m} & 0 \\
0 & \sigma \frac{m}{u}
\end{array}\right], \quad m \in \mathbb{R}^{+},
$$

for both cases $\sigma= \pm 1$. Note that this monodromy matrix is unbounded in the complex $u$-plane. The involution $\downarrow$ acts again as transposition on matrices.

Using the spectral curve (2.13), we obtain

$$
\sigma \frac{u}{m}=-\frac{\rho}{2 m} \frac{\left(\tau-\tau_{0}\right)\left(\tau-\widetilde{\tau}_{0}\right)}{\tau},
$$

where $\tau_{0}=\varphi_{\alpha=0}(\rho, v)$, with $\varphi_{\alpha}(\rho, v)$ given in (8.4),

$$
\tau_{0}=-\sigma \frac{-v+\sqrt{v^{2}+\sigma \rho^{2}}}{\rho}
$$

and where $\widetilde{\tau}_{0}=-\sigma / \tau_{0}$.

Next, let us factorize (9.2) with respect to a contour $\Gamma$ that satisfies Assumption 1, cf. section 6 . There are two possible classes of contours, from which $\Gamma$ can be chosen:

(i) $\tau_{0}$ is inside the contour $\Gamma$ (and hence $\widetilde{\tau}_{0}$ is outside of $\Gamma$ );

(ii) $\widetilde{\tau}_{0}$ is inside the contour $\Gamma$ (and hence $\tau_{0}$ is outside of $\Gamma$ ).

Factorizing with respect to $\Gamma$ we obtain, for each of these cases,

$$
\sigma \frac{u}{m}=\sigma m_{-}(\tau) m_{+}(\tau)
$$

and

$$
M(\rho, v)=\left[\begin{array}{cc}
\sigma m_{-}(\infty) & 0 \\
0 & \sigma m_{-}^{-1}(\infty)
\end{array}\right]=\left[\begin{array}{cc}
\Delta & 0 \\
0 & \Delta^{-1}
\end{array}\right]
$$

where

i) for a contour in class $(i)$

$$
m_{+}(\tau)=\frac{\tau-\widetilde{\tau}_{0}}{-\widetilde{\tau}_{0}}, \quad m_{-}(\tau)=\frac{\sigma \rho}{2 m} \frac{\left(\tau-\tau_{0}\right)}{\tau} \widetilde{\tau}_{0}, \quad \Delta=\frac{\rho \widetilde{\tau}_{0}}{2 m}=\sigma \frac{v+\sqrt{v^{2}+\sigma \rho^{2}}}{2 m}
$$

ii) for a contour in class $(i i)$

$$
m_{+}(\tau)=\frac{\tau-\tau_{0}}{-\tau_{0}}, \quad m_{-}(\tau)=\frac{\sigma \rho}{2 m} \frac{\left(\tau-\widetilde{\tau}_{0}\right)}{\tau} \tau_{0}, \quad \Delta=\frac{\rho \tau_{0}}{2 m}=\sigma \frac{v-\sqrt{v^{2}+\sigma \rho^{2}}}{2 m} .
$$


We take the four-dimensional space-time metric to be given by

$$
d s_{4}^{2}=-\sigma \Delta d t^{2}+\Delta^{-1}\left(e^{\psi} d s_{2}^{2}+\rho^{2} d \phi^{2}\right)
$$

with $d s_{2}^{2}$ given by either (2.5) or (2.6). $\psi$ is obtained by solving (2.7), and we demand $\Delta>0$. Inspection of (9.6) and (9.7) however shows that there are regions in the Weyl upper-half plane where $\Delta<0$. This can be dealt with by changing the overall sign of the monodromy matrix (9.1).

We will now first discuss the case $\sigma=1$, and subsequently the more intricate case $\sigma=-1$.

\section{$9.1 \sigma=1$}

When $\sigma=1$, we have

$$
\tau_{0}=\frac{v-\sqrt{v^{2}+\rho^{2}}}{\rho}, \quad \widetilde{\tau}_{0}=\frac{v+\sqrt{v^{2}+\rho^{2}}}{\rho} .
$$

Case $i)$ : for $\Gamma$ belonging to class $(i)$, we obtain

$$
\begin{aligned}
\Delta_{i}(\rho, v) & =\frac{v+\sqrt{v^{2}+\rho^{2}}}{2 m}>0, \\
\psi_{i}(\rho, v) & =\log \left[\frac{v+\sqrt{v^{2}+\rho^{2}}}{2 \sqrt{v^{2}+\rho^{2}}}\right],
\end{aligned}
$$

up to an integration constant in $\psi_{i}$. The resulting space-time metric takes the form

$$
d s_{4}^{2}=-\frac{\left(v+\sqrt{v^{2}+\rho^{2}}\right)}{2 m} d t^{2}+\frac{m}{\sqrt{v^{2}+\rho^{2}}}\left(d \rho^{2}+d v^{2}\right)+\frac{2 m \rho^{2}}{v+\sqrt{v^{2}+\rho^{2}}} d \phi^{2} .
$$

Now we perform the following change of coordinates,

$$
\rho=\frac{\tilde{\rho}}{2 m} \tilde{z}, \quad v=\frac{1}{4 m}\left(\tilde{z}^{2}-\tilde{\rho}^{2}\right), \quad t=2 m \tilde{t} .
$$

Since $\rho>0$, this requires restricting to either $\tilde{\rho}, \tilde{z} \in] 0, \infty[$ or $\tilde{\rho}, \tilde{z} \in]-\infty, 0[$. We choose $\tilde{\rho}, \tilde{z} \in] 0, \infty[$. Then, (9.11) becomes

$$
d s_{4}^{2}=-\tilde{z}^{2} d \tilde{t}^{2}+d \tilde{z}^{2}+d \tilde{\rho}^{2}+\tilde{\rho}^{2} d \phi^{2},
$$

which describes the Rindler metric, i.e. the uniformly accelerated metric [8].

Now recall that if $M(\rho, v)$ is a diagonal matrix that yields a solution to the field equations, then also $M^{-1}(\rho, v)$ yields a solution to the field equations. The former solution is constructed from $(\Delta, \psi)$, whereas the latter is constructed from $\left(\Delta^{-1}, \psi\right)$, and therefore differ from the former in general. In this latter case, we obtain for the space-time metric,

$$
d s_{4}^{2}=-\frac{2 m}{\left(v+\sqrt{v^{2}+\rho^{2}}\right)} d t^{2}+\frac{\left(v+\sqrt{v^{2}+\rho^{2}}\right)^{2}}{4 m \sqrt{v^{2}+\rho^{2}}}\left(d \rho^{2}+d v^{2}\right)+\frac{\left(v+\sqrt{v^{2}+\rho^{2}}\right)}{2 m} \rho^{2} d \phi^{2} .
$$


Performing the change of coordinates

$$
\rho=\sqrt{m} z \sqrt{r}, \quad v=r-\frac{m}{4} z^{2}
$$

with $r>0, z>0$, the metric becomes

$$
d s_{4}^{2}=-\frac{m}{r} d t^{2}+\frac{r}{m} d r^{2}+r^{2}\left(d z^{2}+z^{2} d \phi^{2}\right),
$$

which is the $A I I I$-metric (C.5) for space-like $r$.

Case $\boldsymbol{i i}$ ): for $\Gamma$ belonging to class $(i i)$, we obtain

$$
\Delta_{i i}(\rho, v)=\frac{v-\sqrt{v^{2}+\rho^{2}}}{2 m}<0,
$$

which requires changing the overall sign of the monodromy matrix (9.1), as mentioned above. The expression for $\psi_{i i}(\rho, v)$ is, up to an integration constant, given by

$$
\psi_{i i}(\rho, v)=\log \left|\frac{v-\sqrt{v^{2}+\rho^{2}}}{2 \sqrt{v^{2}+\rho^{2}}}\right| .
$$

Since the coordinate transformation $v \mapsto-v$ converts the expressions for $\Delta_{i i}$ and $\psi_{i i}$ into those for $\Delta_{i}$ and $\psi_{i}$ given above, the resulting space-time metric agrees with the one of Case $i)$.

A similar reasoning applies to the solution obtained from $M^{-1}(\rho, v)$.

$9.2 \sigma=-1$

When $\sigma=-1$, we have

$$
\tau_{0}=\frac{-v+\sqrt{v^{2}-\rho^{2}}}{\rho}, \quad \widetilde{\tau}_{0}=-\frac{v+\sqrt{v^{2}-\rho^{2}}}{\rho} .
$$

Imposing that $\tau_{0}$ and $\widetilde{\tau}_{0}$ are real, so as to ensure that $\Delta$ is real, requires restricting $(\rho, v)$ to lie in one of the following regions of the Weyl upper-half plane: either $v>\rho$ or $v<-\rho$.

Let us first consider the case when $(\rho, v)$ takes values in the region $v>\rho$.

Case $i$ ): for $\Gamma$ belonging to class $(i)$, we obtain

$$
\Delta_{i}(\rho, v)=\frac{-v-\sqrt{v^{2}-\rho^{2}}}{2 m}<0,
$$

which requires changing the overall sign of the monodromy matrix (9.1), as mentioned above. The expression for $\psi_{i}(\rho, v)$ is, up to an integration constant, given by

$$
\psi_{i}(\rho, v)=\log \left(\frac{v+\sqrt{v^{2}-\rho^{2}}}{2 \sqrt{v^{2}-\rho^{2}}}\right) .
$$

We take the space-time metric to be given by (9.8) with (2.5),

$$
d s_{4}^{2}=\frac{\left(v+\sqrt{v^{2}-\rho^{2}}\right)}{2 m} d t^{2}+\frac{m}{\sqrt{v^{2}-\rho^{2}}}\left(-d \rho^{2}+d v^{2}\right)+\frac{2 m \rho^{2}}{v+\sqrt{v^{2}-\rho^{2}}} d \phi^{2} .
$$


Now we perform the following change of coordinates,

$$
\rho=\frac{\tilde{\rho} \tilde{z}}{2 m}, \quad v=\frac{1}{4 m}\left(\tilde{z}^{2}+\tilde{\rho}^{2}\right), \quad t=2 m \tilde{t} .
$$

Since $\rho>0$, this requires restricting to either $\tilde{\rho}, \tilde{z} \in] 0, \infty[$ or $\tilde{\rho}, \tilde{z} \in]-\infty, 0[$. We choose $\tilde{\rho}, \tilde{z} \in] 0, \infty\left[\right.$. Moreover, the requirement $v>\rho$ implies $(\tilde{z}-\tilde{\rho})^{2}>0$. When $\tilde{z}>\tilde{\rho}$ we obtain

$$
d s_{4}^{2}=\tilde{z}^{2} d \tilde{t}^{2}-d \tilde{\rho}^{2}+d \tilde{z}^{2}+\tilde{\rho}^{2} d \phi^{2},
$$

while for $\tilde{z}<\tilde{\rho}$ we obtain

$$
d s_{4}^{2}=\tilde{\rho}^{2} d \tilde{t}^{2}+d \tilde{\rho}^{2}-d \tilde{z}^{2}+\tilde{z}^{2} d \phi^{2} .
$$

Both these solutions describe a Kasner metric (7.1) with exponents $p_{1}=1, p_{2}=p_{3}=0$, if $t$ is taken to be an angular coordinate, i.e. $0 \leq \tilde{t}<2 \pi$.

As mentioned above, if $M(\rho, v)$ is a diagonal matrix that yields a solution to the field equations, also $M^{-1}(\rho, v)$ yields a solution to the field equations. In this latter case, we take the space-time metric to be given by (9.8) with (2.6),

$$
d s_{4}^{2}=\frac{2 m}{v+\sqrt{v^{2}-\rho^{2}}} d t^{2}+\frac{\left(v+\sqrt{v^{2}-\rho^{2}}\right)^{2}}{4 m \sqrt{v^{2}-\rho^{2}}}\left(d \rho^{2}-d v^{2}\right)+\frac{\left(v+\sqrt{v^{2}-\rho^{2}}\right)}{2 m} \rho^{2} d \phi^{2} .
$$

We perform the change of coordinates

$$
\rho=\sqrt{m} z \sqrt{r}, \quad v=r+\frac{m}{4} z^{2},
$$

with $r>m z^{2} / 4$. We obtain

$$
d s_{4}^{2}=\frac{m}{r} d t^{2}-\frac{r}{m} d r^{2}+r^{2}\left(d z^{2}+z^{2} d \phi^{2}\right),
$$

which is the $A I I I$-metric (C.6) for time-like $r>m z^{2} / 4$.

Case $\mathbf{i i ) :}$ for $\Gamma$ belonging to class $(i i)$, we obtain

$$
\Delta_{i i}(\rho, v)=\frac{-v+\sqrt{v^{2}-\rho^{2}}}{2 m}<0,
$$

which requires changing the overall sign of the monodromy matrix (9.1), as mentioned above. The expression for $\psi_{i i}(\rho, v)$ is, up to an integration constant, given by

$$
\psi_{i i}(\rho, v)=\log \left|\frac{-v+\sqrt{v^{2}-\rho^{2}}}{2 \sqrt{v^{2}-\rho^{2}}}\right| .
$$

We take the space-time metric to be given by (9.8) with (2.5),

$$
d s_{4}^{2}=\frac{\left(v-\sqrt{v^{2}-\rho^{2}}\right)}{2 m} d t^{2}+\frac{m}{\sqrt{v^{2}-\rho^{2}}}\left(-d \rho^{2}+d v^{2}\right)+\frac{2 m \rho^{2}}{v-\sqrt{v^{2}-\rho^{2}}} d \phi^{2} .
$$


We perform the change of coordinates

$$
\rho=\frac{\tilde{\rho} \tilde{z}}{2 m}, \quad v=\frac{1}{2}\left(\tilde{z}^{2}+\tilde{\rho}^{2}\right), \quad t=2 m \tilde{t},
$$

with the choice $\tilde{\rho}, \tilde{z} \in] 0, \infty\left[\right.$. The condition $v>\rho$ becomes $(\tilde{z}-\tilde{\rho})^{2}>0$. When $\tilde{z}>\tilde{\rho}$ we obtain

$$
d s_{4}^{2}=\tilde{\rho}^{2} d \tilde{t}^{2}-d \tilde{\rho}^{2}+d \tilde{z}^{2}+\tilde{z}^{2} d \phi^{2}
$$

while for $\tilde{z}<\tilde{\rho}$ we obtain

$$
d s_{4}^{2}=\tilde{z}^{2} d \tilde{t}^{2}+d \tilde{\rho}^{2}-d \tilde{z}^{2}+\tilde{\rho}^{2} d \phi^{2} .
$$

Both these solutions describe a Kasner metric (7.1) with exponents $p_{1}=1, p_{2}=p_{3}=0$.

Now let us consider $M^{-1}(\rho, v)$. This again requires changing the overall sign of the monodromy matrix. We take the space-time metric to be given by (9.8) with (2.5),

$$
d s_{4}^{2}=\frac{2 m}{v-\sqrt{v^{2}-\rho^{2}}} d t^{2}+\frac{\left(v-\sqrt{v^{2}-\rho^{2}}\right)^{2}}{4 m \sqrt{v^{2}-\rho^{2}}}\left(-d \rho^{2}+d v^{2}\right)+\frac{\left(v-\sqrt{v^{2}-\rho^{2}}\right)}{2 m} \rho^{2} d \phi^{2} .
$$

We perform the change of coordinates

$$
\rho=\sqrt{m} z \sqrt{r}, \quad v=r+\frac{m}{4} z^{2},
$$

with $r<m z^{2} / 4$. We obtain

$$
d s_{4}^{2}=\frac{m}{r} d t^{2}-\frac{r}{m} d r^{2}+r^{2}\left(d z^{2}+z^{2} d \phi^{2}\right),
$$

which is the $A I I I$-metric (C.6) for time-like $r<m z^{2} / 4$. Thus, combining (9.28) with (9.37) results in the $A I I I$-metric (C.6).

Finally, let us consider the case when $(\rho, v)$ takes values in the region $v<-\rho$, or equivalently, $\tilde{v}=-v>\rho$. Noting that

$$
\begin{aligned}
\Delta_{i}(\rho, v) & =\frac{\tilde{v}-\sqrt{\tilde{v}^{2}-\rho^{2}}}{2 m}>0, & \Delta_{i i}(\rho, v) & =\frac{\tilde{v}+\sqrt{\tilde{v}^{2}-\rho^{2}}}{2 m}>0, \\
\psi_{i}(\rho, v) & =\log \left|\frac{-\tilde{v}+\sqrt{\tilde{v}^{2}-\rho^{2}}}{2 \sqrt{\tilde{v}^{2}-\rho^{2}}}\right|, & \psi_{i i}(\rho, v) & =\log \left(\frac{\tilde{v}+\sqrt{\tilde{v}^{2}-\rho^{2}}}{2 \sqrt{\tilde{v}^{2}-\rho^{2}}}\right),
\end{aligned}
$$

we see that the discussion of the case $v<-\rho$ gets mapped to the previous discussion of the case $v>\rho$.

\section{Solutions with two Killing horizons}

In this section we consider the four-dimensional Einstein-Maxwell-dilaton theory that is obtained by Kaluza-Klein reduction of five-dimensional Einstein gravity. We take the monodromy $\mathcal{M}$ that was introduced in [6],

$$
\mathcal{M}(u)=\left(\frac{H_{2}}{H_{1}}\right)^{1 / 3}\left(\begin{array}{ccc}
H_{1} H_{2} & \sqrt{2} H_{1} & -1 \\
-\sqrt{2} H_{1} & -H_{1} / H_{2} & 0 \\
-1 & 0 & 0
\end{array}\right), \quad \operatorname{det} \mathcal{M}=1
$$


where

$$
H_{1}(u)=h_{1}+\frac{Q}{u}, \quad H_{2}(u)=h_{2}+\frac{P}{u} .
$$

Here $Q$ denotes an electric charge, while $P$ denotes a magnetic charge. The constants $h_{1}, h_{2} \in \mathbb{R}$ denote two deformation parameters that are taken to be positive, $h_{1}, h_{2}>0$.

We consider the spectral curve relation (6.1) with $\sigma=1$. When substituting $u$ by the right hand side of (6.1), the resulting matrix $\mathcal{M}_{(\rho, v)}(\tau)$ exhibits 6 simple poles located at the following points in the $\tau$-plane,

$$
\begin{aligned}
\tau_{0 \pm} & =\frac{1}{\rho}\left(v \pm \sqrt{\rho^{2}+v^{2}}\right), \\
\tau_{\tilde{Q} \pm} & =\frac{1}{\rho}\left(v+\tilde{Q} \pm \sqrt{\rho^{2}+(v+\tilde{Q})^{2}}\right), \\
\tau_{\tilde{P} \pm} & =\frac{1}{\rho}\left(v+\tilde{P} \pm \sqrt{\rho^{2}+(v+\tilde{P})^{2}}\right) .
\end{aligned}
$$

Here, $\tilde{Q}$ and $\tilde{P}$ denote the rescaled charges $\tilde{Q}=Q / h_{1}, \quad \tilde{P}=P / h_{2}$. Note that the values (10.3) are all real, and can thus never coincide with the two fixed points $\pm i$ of the involution $\tau \mapsto-1 / \tau$. As a consequence, one can always pick a contour that avoids passing through these simple poles. Note that $\tau_{0+}, \tau_{\tilde{Q}+}, \tau_{\tilde{P}+}>0$, while $\tau_{0-}, \tau_{\tilde{Q}_{-}}, \tau_{\tilde{P}-}<0$. As shown in [6], the canonical factorization of $\mathcal{M}_{(\rho, v)}(\tau)$ with respect to a chosen contour always exists.

There are several contours that one may pick to perform the canonical factorization of $\mathcal{M}_{(\rho, v)}(\tau)$. These various choices of contour will, in general, result in different solutions to the field equations. In [6] a specific contour was chosen, namely the unit circle in the $\tau$-plane. Moreover, the discussion presented there was restricted to a certain region in the Weyl upper-half plane, to ensure that the three poles $\tau_{0-}, \tau_{\tilde{Q}-}, \tau_{\tilde{P}-}$ lie inside the unit circle, and the poles $\tau_{0+}, \tau_{\tilde{Q}+}, \tau_{\tilde{P}+}$ lie outside. This was achieved by taking $\tilde{Q}, \tilde{P}>0$ and restricting to $v>0$. The latter restrictions are a consequence of having chosen the unit circle as a factorization contour. These restrictions are unnecessary: they can be avoided by choosing a contour that encircles the origin and passes through the fixed points $\pm i$ such that $\tau_{0-}, \tau_{\tilde{Q}_{-}}, \tau_{\tilde{P}_{-}}$lie inside the contour. Such a contour always exists, in view of the fact that $\tau_{0-}, \tau_{\tilde{Q}_{-}}, \tau_{\tilde{P}}$ - are real and negative for any choice of charges $\tilde{Q}, \tilde{P}$ and for any $(\rho, v)$ in the Weyl upper-half plane. In the following, we discuss the canonical factorization of $\mathcal{M}_{(\rho, v)}(\tau)$ with respect to such a contour. A similar discussion can be carried out for any other choice of contour, but we will refrain from doing so in this paper. We will follow the notation used in [6] and use superscripts \pm to denote the values (10.3) that lie inside the contour by $\tau^{+}$and the values that lie outside the contour by $\tau^{-}$. Thus, for the choice of contour described above, we set $\tau_{0-}=\tau_{0}^{+}, \tau_{\tilde{Q}_{-}}=\tau_{\tilde{Q}}^{+}, \tau_{\tilde{P}-}=\tau_{\tilde{P}}^{+}$.

The resulting four-dimensional space-time metric takes the form

$$
d s_{4}^{2}=-\Delta(d t+B d \phi)^{2}+\Delta^{-1}\left(e^{\psi}\left(d \rho^{2}+d v^{2}\right)+\rho^{2} d \phi^{2}\right),
$$

where $\Delta, B$ and $e^{\psi}$ are functions of $(\rho, v)$. The solution carries electric-magnetic charges $(Q, P)$, and hence is supported by an electric-magnetic field $F$. It is also supported by a scalar field $e^{-2 \Phi}$. 
We begin by considering the case $\tilde{Q}, \tilde{P}>0$. When $\tilde{Q}=\tilde{P}$, the solution resulting from the canonical factorizaton of $\mathcal{M}_{(\rho, v)}(\tau)$ describes a four-dimensional extremal black hole supported by a constant dilaton field. We therefore take $\tilde{Q} \neq \tilde{P}$. For concreteness, we choose $\tilde{J} \equiv \tilde{P}-\tilde{Q}>0$. The resulting expressions for $\Delta, B, e^{\psi}, F$ and $e^{-2 \Phi}$ are given in appendix A.

As $\rho^{2}+v^{2} \rightarrow \infty$, the solution behaves as follows. It asymptotes to a stationary solution with an effective NUT parameter $\tilde{J}$ which is expressed in terms of the electric-magnetic charges,

$$
\begin{aligned}
d s_{4}^{2}= & -\frac{1}{h_{1} h_{2}}\left(1-\frac{\tilde{P}+\tilde{Q}}{\sqrt{\rho^{2}+v^{2}}}\right)\left(d t-h_{1} h_{2} \tilde{J} \frac{v}{\sqrt{\rho^{2}+v^{2}}} d \phi\right)^{2} \\
& +h_{1} h_{2}\left(1+\frac{\tilde{P}+\tilde{Q}}{\sqrt{\rho^{2}+v^{2}}}\right)\left(d \rho^{2}+d v^{2}+\rho^{2} d \phi^{2}\right)
\end{aligned}
$$

with $e^{-2 \Phi} \rightarrow h_{1} / h_{2}$.

The metric (10.4) has two Killing horizons, $\|\partial / \partial t\|^{2}=\Delta=0$, located at $\rho=v=0$ and at $\rho=0,-\tilde{P}<v<-\tilde{Q}$, respectively. The latter could not be detected in the analysis of [6], because the choice of the unit circle as a factorization contour only allowed to consider the region $v>0$. When approaching the Killing horizon $\rho=v=0$, keeping $\rho / v$ constant, the metric takes the form

$$
d s_{4}^{2}=-\frac{\rho^{2}+v^{2}}{P Q}\left(d t+h_{1} h_{2} \tilde{J} f\left(v / \sqrt{\rho^{2}+v^{2}}\right) d \phi\right)^{2}+\frac{P Q}{\rho^{2}+v^{2}}\left(d \rho^{2}+d v^{2}+\rho^{2} d \phi^{2}\right),
$$

where $f(x)=x(1-x)-1$ denotes a linear combination of the Legendre polynomials $P_{0}, P_{1}$ and $P_{2}$. The scalar field approaches the value $e^{-2 \Phi} \rightarrow P / Q$. Note, however, that $\partial_{\rho, v} e^{-2 \Phi}$ does not vanish at $\rho=v=0$ when $\tilde{J} \neq 0$. Thus, only when $\tilde{J}=0$ (that is, in the extremal black hole case) does the solution exhibit an attractor behaviour as one approaches $\rho=v=0$. Both the Ricci and the Kretschmann scalars are well-behaved at the Killing horizon $\rho=v=0$. However, they both blow up at the Killing horizon $\rho=0,-\tilde{P}<v<-\tilde{Q}$, which points to the existence of a curvature singularity at this horizon. The scalar field $e^{-2 \Phi}$ also diverges at this Killing horizon.

Thus, this solution describes a space-time that is supported by one electric and one magnetic charge and by a scalar field, possesses two Killing horizons and asymptotes to a space-time with an effective NUT parameter $\tilde{J}=\tilde{P}-\tilde{Q}$ that is expressed in terms of the electric-magnetic charges. To the best of our knowledge, this four-dimensional solution has not been given in the literature before. This solution has similarities with a solution discovered by Brill [23] (see also [24]) in a different four-dimensional theory, namely an Einstein+Maxwell theory, in the sense that it possesses two Killing horizons, one of them being associated with the presence of a NUT parameter. However, while Brill's solution describes an electrically charged (or magnetically charged) Reissner-Nordstrom black hole when the NUT parameter is switched off, our solution describes a dyonic extremal black hole solution (that is supported by a scalar field) when the NUT parameter $\tilde{J}$ is set to zero. Moreover, 


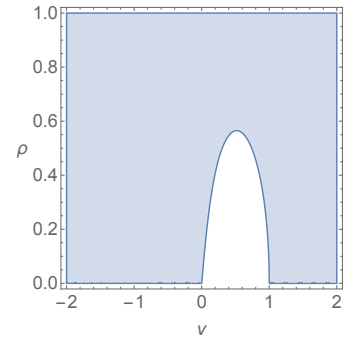

(a) $P=-Q=1$, $h_{1}=h_{2}=1$.

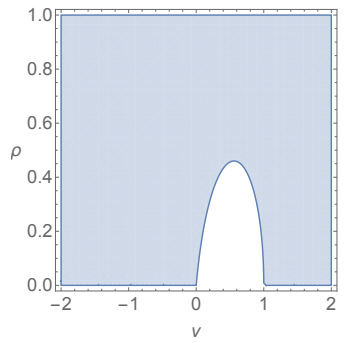

(b) $P=-Q=1$, $h_{1}=1, h_{2}=1 / 9$.

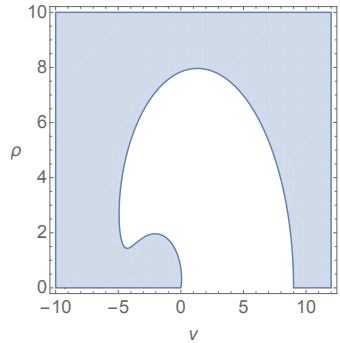

(c) $P=-Q=1$, $h_{1}=1 / 9, h_{2}=1$.

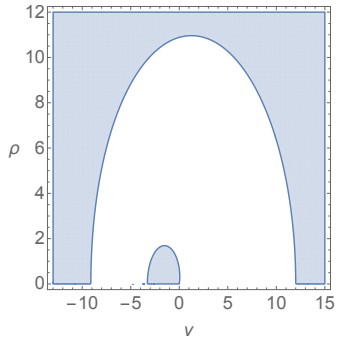

(d) $P=-Q=1$, $h_{1}=1 / 12, h_{2}=1$.

Figure 8. Examples of the curve $e^{2 \Sigma_{2}}=0$ for different values of $\tilde{P}$ and $\tilde{Q}$ with $Q P<0$.

differently from Brill's solution, the NUT parameter $\tilde{J}$ is not an additional parameter, but rather an effective parameter that is expressed in terms of the electric-magnetic charges.

Let us now discuss what happens to the solution when one sends the parameters $h_{1}$ and $h_{2}$ to zero, keeping $Q$ and $P$ fixed. In this limit the solution becomes the $A d S_{2} \times S^{2}$ solution, supported by a constant scalar field $e^{-2 \Phi}=P / Q$, that describes the near-horizon region of the extremal black hole solution.

Next, let us briefly consider the case $\tilde{Q}<0, \tilde{P}>0$. Performing the canonical factorization of $\mathcal{M}_{(\rho, v)}(\tau)$ with respect to the contour described above, results in a solution that, as $\rho^{2}+v^{2} \rightarrow \infty$, asymptotes to a stationary solution with an effective NUT parameter $\tilde{J}=\tilde{P}-\tilde{Q}$, as in the previous case,

$$
\begin{aligned}
d s_{4}^{2}= & -\frac{1}{h_{1} h_{2}}\left(1-\frac{\tilde{P}+\tilde{Q}}{\sqrt{\rho^{2}+v^{2}}}\right)\left(d t-h_{1} h_{2} \tilde{J} \frac{v}{\sqrt{\rho^{2}+v^{2}}} d \phi\right)^{2} \\
& +h_{1} h_{2}\left(1+\frac{\tilde{P}+\tilde{Q}}{\sqrt{\rho^{2}+v^{2}}}\right)\left(d \rho^{2}+d v^{2}+\rho^{2} d \phi^{2}\right)
\end{aligned}
$$

with $e^{-2 \Phi} \rightarrow h_{1} / h_{2}$. Note that now the combination $\tilde{P}+\tilde{Q}$ may be negative. Moreover, unlike in the previous case, the function $\Delta(\rho, v)$ in the metric (10.4) will generically cease to be real in a region of the Weyl upper-half plane. The function $\Delta^{-2}$ is given by $\Delta^{-2}=$ $\left(g m_{1}\right)^{2} e^{2 \Sigma_{2}}$, cf. appendix A. The curve in the Weyl upper-half plane where $e^{2 \Sigma_{2}}$ vanishes determines the boundary where $\Delta$ ceases to be real. Note that this boundary does not coincide with the axis $\rho=0$, and that the Ricci and the Kretschmann scalars blow up when approaching this boundary. See figure 8 for examples of the curve in the Weyl upper-half plane where $e^{2 \Sigma_{2}}$ vanishes.

Finally, let us discuss what happens to the solution when one sends the parameters $h_{1}$ and $h_{2}$ to zero, keeping $Q$ and $P$ fixed. For the various function in the line element (10.4) we obtain

$$
e^{\psi}=\rho^{\frac{4}{9}}, \quad B=2 P Q \frac{v-\sqrt{\rho^{2}+v^{2}}}{\rho^{2}+v^{2}}+f_{1}, \quad \Delta=-\frac{\rho^{2}+v^{2}}{P Q} \frac{\rho}{\sqrt{\rho^{2}+4 v\left(v-\sqrt{\rho^{2}+v^{2}}\right)}},
$$


where $f_{1}$ denotes an integration constant. When solving the differential equations (A.3) for $\psi$, we took the resulting integration constant to equal

$$
c_{1}=\frac{4}{9} \log \left[\frac{2 \tilde{P} \tilde{Q}}{\tilde{Q}-\tilde{P}}\right],
$$

to ensure that the differential equations (A.3) remain well-defined in the limit $h_{1}, h_{2} \rightarrow 0$. Note that when $v>0, \Delta$ is only real for $\rho \geq 2 \sqrt{2} v$.

Thus, the case $\tilde{Q}<0, \tilde{P}>0$ is markedly different from the case $\tilde{Q}>0, \tilde{P}>0$ discussed earlier.

\section{Acknowledgments}

We would like to thank Thomas Mohaupt and Suresh Nampuri for useful discussions. M.C. Câmara and G.L. Cardoso thank David Krejčiřík for hospitality at the Department of Mathematics, Czech Technical University Prague, during the course of this work. This work was partially supported by FCT/Portugal through UID/MAT/04459/2019 and through the LisMath PhD fellowships PD/BD/128415/2017 (P. Aniceto) and PD/BD/135527/2018 (M. Rosselló).

\section{A Expressions for $\tilde{\boldsymbol{P}}>\tilde{\boldsymbol{Q}}>0$}

We give the expressions for the solution with $\tilde{P}>\tilde{Q}>0$ discussed in section 10 .

We introduce the quantities [6],

$$
\begin{aligned}
g & =\left(\frac{h_{2}}{h_{1}}\right)^{1 / 3}\left(\frac{\tau_{\tilde{P}}^{-}}{\tau_{\tilde{Q}}^{-}}\right)^{1 / 3}, \\
m_{1} & =h_{1} h_{2}\left(1-\frac{2 \tilde{Q}}{\rho\left(\tau_{0}^{+}-\tau_{0}^{-}\right)}\right)\left(1-\frac{2 \tilde{P}}{\rho\left(\tau_{0}^{+}-\tau_{0}^{-}\right)}\right)-2 h_{1} h_{2} \frac{\left(\tau_{\tilde{Q}}^{+}-\tau_{\tilde{P}}^{+}\right)\left(\tau_{0}^{+}-\tau_{\tilde{P}}^{-}\right)\left(\tau_{0}^{+}-\tau_{\tilde{Q}}^{+}\right)}{\tau_{\tilde{Q}}^{+}\left(\tau_{0}^{+}-\tau_{0}^{-}\right)^{2}}, \\
m_{2} & =\sqrt{2} h_{1}\left(1-\frac{2 \tilde{Q}}{\rho\left(\tau_{0}^{+}-\tau_{0}^{-}\right)}\right)-\sqrt{2} h_{1} \frac{\left(\tau_{\tilde{Q}}^{+}-\tau_{\tilde{P}}^{+}\right)\left(\tau_{0}^{+}-\tau_{\tilde{Q}}^{+}\right)}{\tau_{\tilde{Q}}^{+}\left(\tau_{0}^{+}-\tau_{0}^{-}\right)}, \\
m_{3} & =-\frac{h_{1}}{h_{2}} \frac{\tau_{\tilde{Q}}^{-}}{\tau_{\tilde{P}}^{-}}=-\frac{1}{g^{3}}, \\
\chi_{1} & =-m_{2} /\left(m_{1} m_{3}+\left(m_{2}\right)^{2}\right), \quad \chi_{2}=m_{2} / m_{1}, \quad \chi_{3}=-1 / m_{1}, \\
e^{2 \Sigma_{1}} & =m_{1} g, \quad e^{2 \Sigma_{2}}=\left(m_{3}+\left(m_{2}\right)^{2} / m_{1}\right) g .
\end{aligned}
$$

Then $[6]$

$$
\begin{aligned}
\Delta^{-2} & =g^{3} m_{1}\left(m_{1} m_{3}+\left(m_{2}\right)^{2}\right), \\
e^{-2 \Phi} & =g^{3 / 2}\left(m_{3}+\frac{\left(m_{2}\right)^{2}}{m_{1}}\right)^{3 / 2}
\end{aligned}
$$


In [6], the expressions for $\psi$ and for $B$ were studied by taking $\tilde{J}=\tilde{P}-\tilde{Q}$ to be small and performing a power series expansion in $\tilde{J}$. Only the first few terms in these expansions were given. Here we give the exact expressions which, when power expanded in $\tilde{J}$, reproduce the results given in [6]. $\psi$ is obtained by integrating

$$
\begin{aligned}
& \partial_{\rho} \psi=\frac{1}{4} \rho g^{4}\left[\left(\partial_{\rho}\left(g m_{3}\right)\right)^{2}-\left(\partial_{v}\left(g m_{3}\right)\right)^{2}\right], \\
& \partial_{v} \psi=\frac{1}{2} \rho g^{4} \partial_{\rho}\left(g m_{3}\right) \partial_{v}\left(g m_{3}\right) .
\end{aligned}
$$

We obtain

$$
\psi=\frac{1}{9} \log \left[\frac{\left(\rho^{2}+(\tilde{P}+v)(\tilde{Q}+v)+\sqrt{\rho^{2}+(\tilde{P}+v)^{2}} \sqrt{\rho^{2}+(\tilde{Q}+v)^{2}}\right)^{2}}{4\left(\rho^{2}+(\tilde{P}+v)^{2}\right)\left(\rho^{2}+(\tilde{Q}+v)^{2}\right)}\right]+c_{1},
$$

with $c_{1}$ an integration constant that we set to zero to ensure that in the limit $\tilde{J}=\tilde{P}-\tilde{Q}=0$ we obtain $\psi=0$.

$B$ is determined by solving for the two-form $\mathcal{F}=d(B d \phi)$,

$$
-e^{-2 \phi_{2}} * \mathcal{F}=d \chi_{3}-\chi_{1} d \chi_{2} .
$$

Here, the dual $*$ is taken with respect to the three-dimensional metric

$$
d s_{3}^{2}=e^{\psi}\left(d \rho^{2}+d v^{2}\right)+\rho^{2} d \phi^{2} .
$$

Adjusting the integration constant, we obtain the following expression for $B$,

$$
B=h_{1} h_{2} \frac{\left(\tilde{Q} \sqrt{\rho^{2}+(\tilde{P}+v)^{2}}-\tilde{P} \sqrt{\rho^{2}+(\tilde{Q}+v)^{2}}\right)\left(v-\sqrt{\rho^{2}+v^{2}}\right)}{\rho^{2}+v^{2}}+h_{1} h_{2}(\tilde{Q}-\tilde{P}) .
$$

In the limit $\tilde{J}=\tilde{P}-\tilde{Q}=0$ we obtain $B=0$.

The above expressions completely determine the space-time metric (10.4) and the scalar field $e^{-2 \Phi}$ that supports the solution. The solution is further supported by an electric-magnetic field $F=d A^{0}$, where the one-form $A^{0}$ is given by

$$
A^{0}=\chi_{1} d t+A_{\phi} d \phi
$$

with $A_{\phi}$ determined by solving

$$
\begin{aligned}
& \partial_{\rho} A_{\phi}=-e^{2\left(\Sigma_{1}-\Sigma_{2}\right)} \rho \partial_{v} \chi_{2}+B \partial_{\rho} \chi_{1} \\
& \partial_{v} A_{\phi}=e^{2\left(\Sigma_{1}-\Sigma_{2}\right)} \rho \partial_{\rho} \chi_{2}+B \partial_{v} \chi_{1} .
\end{aligned}
$$

In the following, we solve these partial differential equations by means of a power series in $\tilde{J}$

$$
A_{\phi}=\sum_{n=0}^{\infty} A_{\phi}^{(n)} \frac{\tilde{J}^{n}}{n !}
$$


In [6] $A_{\phi}$ was given up to first order in $\tilde{J}$ only. Here we determine $A_{\phi}$ up to second order in $\tilde{J}$,

$$
\begin{aligned}
& A_{\phi}^{(0)}=\frac{\sqrt{2} h_{2} \tilde{Q} v}{\sqrt{\rho^{2}+v^{2}}}, \\
& A_{\phi}^{(1)}=\frac{h_{2}}{\sqrt{2}}\left(\frac{v}{\sqrt{\rho^{2}+v^{2}}}+\frac{\left(v-\sqrt{\rho^{2}+v^{2}}\right) \sqrt{\rho^{2}+(\tilde{Q}+v)^{2}}}{\rho^{2}+v^{2}+\tilde{Q} \sqrt{\rho^{2}+v^{2}}}-\frac{2 \tilde{Q}}{\tilde{Q}+\sqrt{\rho^{2}+v^{2}}}\right) \\
& A_{\phi}^{(2)}=\frac{h_{2}}{2 \sqrt{2}}\left(\frac{2 v^{2}\left(\tilde{Q}+4 v-\sqrt{\rho^{2}+(\tilde{Q}+v)^{2}}\right)}{\tilde{Q}^{2}(\tilde{Q}+2 v) \sqrt{\rho^{2}+v^{2}}}-\frac{4\left[(\tilde{Q}+v)^{2}+\tilde{Q} \sqrt{\rho^{2}+(\tilde{Q}+v)^{2}}\right]}{\left(\tilde{Q}+\sqrt{\rho^{2}+v^{2}}\right)^{3}}\right. \\
& -\frac{4\left(2 \tilde{Q}^{2}+v^{2}\right)+(\tilde{Q}-v) \sqrt{\rho^{2}+(\tilde{Q}+v)^{2}}}{\tilde{Q}^{2}\left(\tilde{Q}+\sqrt{\rho^{2}+v^{2}}\right)} \\
& +\frac{2\left[-2 v^{2}+(3 \tilde{Q}+v)\left(2 \tilde{Q}+\sqrt{\rho^{2}+(\tilde{Q}+v)^{2}}\right)\right]}{\tilde{Q}\left(\tilde{Q}+\sqrt{\rho^{2}+v^{2}}\right)^{2}} \\
& \left.-\frac{(\tilde{Q}+v)\left[\left(\tilde{Q}+2 v-\sqrt{\rho^{2}+v^{2}}\right) \sqrt{\rho^{2}+(\tilde{Q}+v)^{2}}-2(\tilde{Q}+v) \sqrt{\rho^{2}+v^{2}}\right]}{\tilde{Q}(\tilde{Q}+2 v)\left[\rho^{2}+(\tilde{Q}+v)^{2}\right]}\right] .
\end{aligned}
$$

\section{B Gluing solutions along the lines $\rho= \pm v+m$}

The extensions (8.49) exhibit a jump in the transverse extrinsic curvature across the lines $\rho= \pm v+m$, as we now verify for the first extension in (8.49). We consider the gluing of the solution based on $\Delta_{i}(\rho, v)$ in region $I$ with the solution based on $\Delta_{i}\left(B^{-1}(\rho, v)\right)$ in region $I I$ along the hypersurface given by $\rho=m-v$. We recall that demanding $\rho$ to be a time-like coordinate in both regions $I$ and $I I$ requires the transformation $d s_{2}^{2} \rightarrow-d s_{2}^{2}$ when passing from region $I$ to region $I I$. Using the bijections given in table 2, the associated space-time metrics read in spherical coordinates,

$$
\begin{aligned}
g_{I} & =\left(\frac{2 m}{r}-1\right) d t^{2}-\frac{1}{\frac{2 m}{r}-1} d r^{2}+r^{2}\left(d \theta^{2}+\sin ^{2} \theta d \phi^{2}\right) \\
g_{I I} & =\tan ^{2}\left(\frac{\theta}{2}\right) d t^{2}-4 m^{2} \cos ^{4}\left(\frac{\theta}{2}\right)\left(\frac{d r^{2}}{r(2 m-r)}-d \theta^{2}-\frac{r(2 m-r)}{m^{2}} d \phi^{2}\right) .
\end{aligned}
$$

The gluing of these solutions is performed along the hypersurface $\Sigma$ given by $r=m+m \cos \theta$ with $0<\theta<\pi / 2$. The metric is continuous at $\Sigma$, i.e. $g_{\left.I\right|_{T \Sigma}}=g_{\left.I I\right|_{T \Sigma}}$. There is, however, a jump in the transverse extrinsic curvature [12, 25], as follows. 
To study the jump in the transverse extrinsic curvature when traversing $\Sigma$, we begin by considering the 'normal' vector field $k$ to this hypersurface, evaluated on $\Sigma$, given by

$$
\left.k^{b}\right|_{\Sigma}=\left.k_{\mu} d x^{\mu}\right|_{\Sigma}=\left.(d(r-m-m \cos \theta))\right|_{\Sigma}=\left.(d r+\sqrt{(2 m-r) r} d \theta)\right|_{\Sigma} .
$$

Since $\|k\|_{\Sigma}=0$, the vector field $k$ is also tangent to $\Sigma$, and so are the vector fields $\partial_{A}$ with $A=t, \phi$. A null transverse vector field $\ell$, i.e. a vector field satisfying $\|\ell\|_{\Sigma}=0,(\ell \cdot k)_{\Sigma}=$ $-1,\left(\ell \cdot \partial_{A}\right)_{\Sigma}=0$, is then given by

$$
\ell=\ell^{\mu} \partial_{\mu}=-\frac{1}{2} \partial_{r}-\frac{1}{2 \sqrt{(2 m-r) r}} \partial_{\theta} .
$$

The jump in the transverse extrinsic curvature is computed using $\ell$ [12, 25], and given by

$$
\frac{1}{2} £_{\ell} g_{\left.I I\right|_{T \Sigma}}-\frac{1}{2} £_{\ell} g_{\left.I\right|_{T \Sigma}}=-\frac{m}{r} d t^{2}+\frac{(2 m-r) r^{2}}{m^{2}} d \phi^{2},
$$

which is non-vanishing. Here, $£_{\ell}$ denotes the Lie derivative with respect to the vector field $\ell$.

The jump in the transverse extrinsic curvature is associated with the presence of a discontinuity of the Weyl tensor $[12,13]$; across $\Sigma$, the metric is only continuous.

\section{C $A$-metrics}

Let $m>0$. The class of $A$-metrics comprises the following space-time metrics in four dimensions $[7,8]$ :

1. AI-metrics:

the Schwarzschild solution (with $0<r<2 m$ or $2 m<r<\infty, 0 \leq \phi<2 \pi, 0<\theta<\pi$ )

$$
d s_{4}^{2}=-\left(1-\frac{2 m}{r}\right) d t^{2}+\left(1-\frac{2 m}{r}\right)^{-1} d r^{2}+r^{2}\left(d \theta^{2}+\sin ^{2} \theta d \phi^{2}\right),
$$

and the 'negative mass' Schwarzschild solution (with $0<r<\infty, 0 \leq \phi<2 \pi, 0<$ $\theta<\pi)$

$$
d s_{4}^{2}=-\left(1+\frac{2 m}{r}\right) d t^{2}+\left(1+\frac{2 m}{r}\right)^{-1} d r^{2}+r^{2}\left(d \theta^{2}+\sin ^{2} \theta d \phi^{2}\right) .
$$

2. AII-metrics:

the solution (with $0<\varrho<2 m$ or $2 m<\varrho<\infty, 0 \leq \phi<2 \pi, 0<\vartheta<\infty$ )

$$
d s_{4}^{2}=\left(1-\frac{2 m}{\varrho}\right) d t^{2}-\left(1-\frac{2 m}{\varrho}\right)^{-1} d \varrho^{2}+\varrho^{2}\left(d \vartheta^{2}+\sinh ^{2} \vartheta d \phi^{2}\right)
$$

and the solution (with $0<\varrho<\infty, 0 \leq \phi<2 \pi, 0<\vartheta<\infty$ )

$$
d s_{4}^{2}=\left(1+\frac{2 m}{\varrho}\right) d t^{2}-\left(1+\frac{2 m}{\varrho}\right)^{-1} d \varrho^{2}+\varrho^{2}\left(d \vartheta^{2}+\sinh ^{2} \vartheta d \phi^{2}\right) .
$$


3. AIII-metrics:

the $r$ space-like solution (with $m>0,0<r<\infty, 0 \leq \phi<2 \pi, 0<\rho<\infty$ )

$$
d s_{4}^{2}=-\frac{2 m}{r} d t^{2}+\frac{r}{2 m} d r^{2}+r^{2}\left(d \rho^{2}+\rho^{2} d \phi^{2}\right)
$$

and the $r$ time-like solution

$$
d s_{4}^{2}=\frac{2 m}{r} d t^{2}-\frac{r}{2 m} d r^{2}+r^{2}\left(d \rho^{2}+\rho^{2} d \phi^{2}\right) .
$$

These solutions can be transformed, respectively, to the solution (with $0<x_{2},-\infty<$ $\left.t, x_{1}, x_{3}<\infty\right)$

$$
d s_{4}^{2}=-x_{2}^{-2 / 3} d t^{2}+d x_{2}^{2}+x_{2}^{4 / 3}\left(d x_{1}^{2}+d x_{3}^{2}\right),
$$

and to the Kasner solution (with $0<t,-\infty<x_{1}, x_{2}, x_{3}<\infty$ )

$$
d s_{4}^{2}=-d t^{2}+t^{-2 / 3} d x_{2}^{2}+t^{4 / 3}\left(d x_{1}^{2}+d x_{3}^{2}\right) .
$$

These space-time metrics arise as follows from factorization.

$\boldsymbol{A I}, \boldsymbol{A I I}$-metrics: the solution describing the exterior region of the Schwarzschild black hole $(r>2 m)$, the 'negative mass' Schwarzschild solution and the solution describing the interior region of (C.3) $(0<\varrho<2 m)$ are obtained by canonical factorization of the Schwarzschild monodromy matrix (8.1) with $\sigma=1$. The solution describing the interior region of the Schwarzschild black hole $(0<r<2 m)$, the solution describing the exterior region of (C.3) $(\varrho>2 m)$ and the solution (C.4) are obtained by canonical factorization of the Schwarzschild monodromy matrix (8.1) with $\sigma=-1$.

AIII-metrics: the space-time metrics (C.5) and (C.6) are obtained by canonical factorization of the monodromy matrix (9.1) with $\sigma=1$ and $\sigma=-1$, respectively.

Open Access. This article is distributed under the terms of the Creative Commons Attribution License (CC-BY 4.0), which permits any use, distribution and reproduction in any medium, provided the original author(s) and source are credited.

\section{References}

[1] G.A. Alekseev, Thirty years of studies of integrable reductions of Einstein's field equations, in On recent developments in theoretical and experimental general relativity, astrophysics and relativistic field theories. Proceedings, $12^{\text {th }}$ Marcel Grossmann meeting on general relativity, Paris, France, 12-18 July 2009, World Scientific, Singapore (2010), pg. 645 [arXiv: 1011.3846] [INSPIRE].

[2] P. Breitenlohner and D. Maison, On the Geroch group, Ann. Inst. H. Poincaré Phys. Theor. 46 (1987) 215.

[3] H. Nicolai, Two-dimensional gravities and supergravities as integrable system, Lect. Notes Phys. 396 (1991) 231 [INSPIRE].

[4] D. Katsimpouri, A. Kleinschmidt and A. Virmani, Inverse scattering and the Geroch group, JHEP 02 (2013) 011 [arXiv: 1211.3044] [INSPIRE].

[5] M.C. Camara, G.L. Cardoso, T. Mohaupt and S. Nampuri, A Riemann-Hilbert approach to rotating attractors, JHEP 06 (2017) 123 [arXiv: 1703.10366] [INSPIRE]. 
[6] G.L. Cardoso and J.C. Serra, New gravitational solutions via a Riemann-Hilbert approach, JHEP 03 (2018) 080 [arXiv:1711.01113] [INSPIRE].

[7] J. Ehlers and W. Kundt, Exact solutions of the gravitational field equations, in Gravitation: an introduction to current research, L. Witten ed., Wiley, U.S.A. (1962), pg. 49.

[8] J.B. Griffiths and J. Podolsky, Exact space-times in Einstein's general relativity, Cambridge Monographs on Mathematical Physics, Cambridge University Press, Cambridge, U.K. (2009)

[9] N. Wiener and E. Hopf, Über eine Klasse singulärer Integralgleichungen (in German), S.-B. Preuss. Akad. Wiss. Berlin Phys. Math. Kl. 30/32 (1931) 696.

[10] K. Clancey and I. Gohberg, Factorization of matrix functions and singular integral operators, in Operator theory: advances and applications, volume 3, Birkhäuser Verlag, Basel, Switzerland (1981).

[11] F.-O. Speck, General Wiener-Hopf factorization methods, Res. Notes Math. 119 (1985) 1.

[12] C. Barrabes and W. Israel, Thin shells in general relativity and cosmology: the lightlike limit, Phys. Rev. D 43 (1991) 1129 [INSPIRE].

[13] C. Barrabes and P. Hogan, Singular null hypersurfaces in general relativity, World Scientific, Singapore (2003).

[14] G. Jones and J.E. Wang, Weyl card diagrams and new S-brane solutions of gravity, hep-th/0409070 [INSPIRE].

[15] G.C. Jones and J.E. Wang, Weyl card diagrams, Phys. Rev. D 71 (2005) 124019 [hep-th/0506023] [INSPIRE].

[16] J.H. Schwarz, Classical symmetries of some two-dimensional models coupled to gravity, Nucl. Phys. B 454 (1995) 427 [hep-th/9506076] [INSPIRE].

[17] H. Lü, M.J. Perry and C.N. Pope, Infinite-dimensional symmetries of two-dimensional coset models coupled to gravity, Nucl. Phys. B 806 (2009) 656 [arXiv:0712.0615] [INSPIRE].

[18] A.R. Its, The Riemann-Hilbert problem and integrable systems, Not. Amer. Math. Soc. 50 (2003) 1389.

[19] G. Litvinchuk and I. Spitkovsky, Factorization of measurable matrix functions, in Oper. Theory Adv. Appl. 25, Birkhäuser Verlag, Basel, Switzerland (1987).

[20] S. Mikhlin and S. Prössdorf, Singular integral operators, Springer-Verlag, Berlin, Germany (1986).

[21] M.C. Câmara, A.B. Lebre and F.-O. Speck, Meromorphic factorization, partial index estimates and elastodynamic diffraction problems, Math. Nachr. 157 (1992) 291.

[22] M.C. Câmara, Toeplitz operators and Wiener-Hopf factorisation: an introduction, Concrete Oper. 4 (2017) 130.

[23] D.R. Brill, Electromagnetic fields in a homogeneous, nonisotropic universe, Phys. Rev. 133 (1964) B845.

[24] H. Stephani, D. Kramer, M. MacCallum, C. Hoenselaers and E. Herlt, Exact solutions of Einstein's field equations, Cambridge Monographs on Mathematical Physics, Cambridge University Press, Cambridge, U.K. (2003).

[25] E. Poisson, A relativist's toolkit: the mathematics of black-hole mechanics, Cambridge University Press, Cambridge, U.K. (2009). 